\title{
INTEGRABLE SYSTEMS, TORIC DEGENERATIONS AND OKOUNKOV BODIES
}

\author{
MEGUMI HARADA AND KIUMARS KAVEH \\ To Askold Georgevich Khovanskii
}

\begin{abstract}
Let $X$ be a smooth projective variety of dimension $n$ over $\mathbb{C}$ equipped with a very ample Hermitian line bundle $\mathcal{L}$. In the first part of the paper, we show that if there exists a toric degeneration of $X$ satisfying some natural hypotheses (which are satisfied in many settings), then there exists a surjective continuous map from $X$ to the special fiber $X_{0}$ which is a symplectomorphism on an open dense subset $U$. From this we are then able to construct a completely integrable system on $X$ in the sense of symplectic geometry. More precisely, we construct a collection of real-valued functions $\left\{H_{1}, \ldots, H_{n}\right\}$ on $X$ which are continuous on all of $X$, smooth on an open dense subset $U$ of $X$, and pairwise Poisson-commute on $U$. Moreover, our integrable system in fact generates a Hamiltonian torus action on $U$. In the second part, we show that the toric degenerations arising in the theory of Newton-Okounkov bodies satisfy all the hypotheses of the first part of the paper. In this case the image of the 'moment map' $\mu=\left(H_{1}, \ldots, H_{n}\right): X \rightarrow \mathbb{R}^{n}$ is precisely the Newton-Okounkov body $\Delta=\Delta(R, v)$ associated to the homogeneous coordinate ring $R$ of $X$, and an appropriate choice of a valuation $v$ on $R$. Our main technical tools come from algebraic geometry, differential (Kähler) geometry, and analysis. Specifically, we use the gradient-Hamiltonian vector field, and a subtle generalization of the famous Łojasiewicz gradient inequality for real-valued analytic functions. Since our construction is valid for a large class of projective varieties $X$, this manuscript provides a rich source of new examples of integrable systems. We discuss concrete examples, including elliptic curves, flag varieties of arbitrary connected complex reductive groups, spherical varieties, and weight varieties.
\end{abstract}

\section{Contents}

Introduction

Part 1. Integrable systems from toric degenerations 7

1. Preliminaries for Part 1

2. Gradient-Hamiltonian flow 9

3. Critical points of the family 10

4. Continuity of the gradient-Hamiltonian flow 12

5. Construction of an integrable system from a toric degeneration 14

6. Integrable systems and (GIT and symplectic) quotients

Part 2. Toric degenerations from valuations and Okounkov bodies 20

Key words and phrases. Okounkov body, toric degeneration, integrable system.

The first author is partially supported by an NSERC Discovery Grant, an NSERC University Faculty Award, and an Ontario Ministry of Research and Innovation Early Researcher Award.

The second author is partially supported by a Simons Foundation Collaboration Grants for Mathematicians and a National Science Foundation Grant. 
7. Preliminaries for Part $2 \quad 20$

8. A toric degeneration associated to a valuation $\quad 23$

9. A Kähler structure on the family $\mathfrak{X} \quad$ 26]

10. Integrable systems constructed from a valuation 33

11. GIT and symplectic quotients 34

12. Examples

References

\section{INTRODUCTION}

A (completely) integrable system on a symplectic manifold is a Hamiltonian system which admits a maximal number of first integrals, also called "conservation laws." A first integral is a function which is constant along the Hamiltonian flow; when there are a maximal number of such, then one can describe the integral curves of the Hamiltonian vector field implicitly by setting the first integrals equal to constants. In this sense an integrable system is very well-behaved. For a recent overview of this subject, see PeVu11 and its extensive bibliography. The theory of integrable systems in symplectic geometry is rather dominated by specific examples (e.g. "spinning top," "Calogero-Moser system," "Toda lattice"). The main contribution of this manuscript, summarized in Theorems A, B below, is a construction of an integrable system on (an open dense subset of) a wide class of complex projective varieties. Moreover, we additionally show that the integrable system in fact generates a Hamiltonian torus action on an open dense subset of the variety. Much is true about Hamiltonian torus actions that is false for integrable systems in general.

Our construction represents a significant expansion of the possible areas of application of the theory of integrable systems and Hamiltonian torus actions, including: complex algebraic geometry, symplectic topology, representation theory, and Schubert calculus, to name just a few. We therefore expect our manuscript to be of wide-ranging interest. Moreover, we use a rather broad mix of techniques coming from algebraic geometry, differential (Kähler) geometry, and analysis, in order to prove our main results. With this in mind, we have attempted to make our exposition accessible to a broad audience.

Throughout, our algebraic varieties are over $\mathbb{C}$.

We begin with a definition; for details see e.g. Au96. Let $(M, \omega)$ be a symplectic manifold of real dimension $2 n$. Let $\left\{f_{1}, f_{2}, \ldots, f_{n}\right\}$ be smooth functions on $M$. Then (by slight abuse of terminology, see Section 1) we call the functions $\left\{f_{1}, \ldots, f_{n}\right\}$ a (completely) integrable system on $M$ if they satisfy the following conditions:

- they pairwise Poisson-commute, i.e. $\left\{f_{i}, f_{j}\right\}=0$ for all $i, j$, where $\{\cdot, \cdot\}$ denotes the Poisson bracket on $\mathcal{C}^{\infty}(M)$ induced from $\omega$, and

- they are functionally independent, i.e. their derivatives $d f_{1}, \ldots, d f_{n}$ are linearly independent almost everywhere on $M$.

In fact, below we will state and use a slightly generalized version of this definition (cf. Definition 1.1.

We recall two examples which may be familiar to researchers outside of symplectic geometry.

Example 1. A smooth projective toric variety $X$ is a symplectic manifold, equipped with the pullback of the standard Fubini-Study form on projective space. The (compact) torus action on $X$ is in fact Hamiltonian in the sense of symplectic geometry, and its moment 
map image is precisely the polytope corresponding to $X$. The torus has real dimension $n=\frac{1}{2} \operatorname{dim}_{\mathbb{R}}(X)$, and its $n$ components form an integrable system on $X$.

Example 2. Let $X=\operatorname{GL}(n, \mathbb{C}) / B$ be the flag variety of nested subspaces in $\mathbb{C}^{n}$. For $\lambda$ a regular highest weight, consider the usual Plücker embedding $X \hookrightarrow \mathbb{P}\left(V_{\lambda}\right)$ where $V_{\lambda}$ denotes the irreducible representation of $\operatorname{GL}(n, \mathbb{C})$ with highest weight $\lambda$. Equip $X$ with the KostantKirillov-Souriau symplectic form coming from its identification with a coadjoint orbit of $U(n, \mathbb{C})$, which meets the positive Weyl chamber at precisely $\lambda$. Then Guillemin-Sternberg GuSt83. build an integrable system, frequently called the Gel'fand-Cetlin integrable system, on $X$ by viewing this coadjoint orbit as a subset of the space of Hermitian $n \times n$ matrices. This integrable system is intimately related to the well-known Gel'fand-Cetlin basis for the irreducible representation $V_{\lambda}$ GeCe50].

Example 2 in fact motivated this paper. Several years ago, we had the idea to use the toric degeneration results for flag varieties given in Gonciulea-Lakshmibai GoLa96, Kogan-Miller KoMi05, and Caldero Ca02, in order to construct integrable systems for flag varieties of general reductive groups, thus generalizing the Gel'fand-Cetlin integrable system constructed by Guillemin and Sternberg. Allen Knutson also had very similar ideas, according to his post on MathOverflow in response to a question from David Treumann KnTr-MO. (His post is dated January 2010, but he apparently had the ideas already in the mid-90's.) More formally, a connection between toric degenerations and the Gel'fand-Cetlin integrable system was established in detail in 2010 in NiNoUe10, using Ruan's technique of gradient-Hamiltonian flows. Their ideas inspired us to prove the results contained in the present paper, which in fact deals not only with flag varieties of general reductive groups, but with a much more general class of projective varieties.

Motivated by the above examples, we make the following definition of a (completely) integrable system on a projective variety which differs slightly from some other definitions in the literature (for more details see Section 1).

Definition 1. Let $X$ be an algebraic variety of dimension $n$. Let $\omega$ be a symplectic form on the smooth locus of $X$. We call a collection of real-valued functions $\left\{F_{1}, \ldots, F_{n}\right\}$ on $X$ a completely integrable system (or an integrable system) on $X$ if the following are satisfied:

(a) $F_{1}, \ldots, F_{n}$ are continuous on all of $X$.

(b) There exists an open dense subset $U$ contained in the smooth locus of $X$ such that $F_{1}, \ldots, F_{n}$ are differentiable on $U$, and the differentials $d F_{1}, \ldots, d F_{n}$ are linearly independent on $U$.

(c) $F_{1}, \ldots, F_{n}$ pairwise Poisson-commute on $U$.

Recall that a special case of an integrable system is given by (the components of) a moment map for a Hamiltonian torus action on a symplectic manifold (see Section 1 for details). Motivated by this, in this manuscript we call the map $\mu:=\left(F_{1}, \ldots, F_{n}\right): X \rightarrow \mathbb{R}^{n}$, constructed from the functions $F_{i}$ above, the moment map of the integrable system.

The main purpose of this paper is to construct integrable systems, in the sense of the definition above, on a large class of projective algebraic varieties. Our main tool is a toric degeneration, in a sense we make precise below. Let $X$ be an $n$-dimensional projective variety.

Definition 2. We call an algebraic family $\pi: \mathfrak{X} \rightarrow \mathbb{C}$ a toric degeneration of $X$ if the following hold: 
(1) $\pi: \mathfrak{X} \rightarrow \mathbb{C}$ is a flat family of irreducible varieties. In particular, each fiber is an irreducible reduced scheme.

(2) The family is trivial over $\mathbb{C}^{*}=\mathbb{C} \backslash\{0\}$ with fiber isomorphic to $X$; more precisely, there exists a fiber-preserving isomorphism $\rho: X \times \mathbb{C}^{*} \rightarrow \pi^{-1}\left(\mathbb{C}^{*}\right)$ from the trivial fiber bundle $X \times \mathbb{C}^{*} \rightarrow \mathbb{C}^{*}$ to $\pi^{-1}\left(\mathbb{C}^{*}\right)$. In other words, $\mathfrak{X} \backslash X_{0}$ is equipped with a $\mathbb{C}^{*}$-action, and the map $\pi$ is $\mathbb{C}^{*}$-equivariant (where $\mathbb{C}^{*}$ acts on the base $\mathbb{C}$ by multiplication).

(3) There is an action of $\mathbb{T}=\left(\mathbb{C}^{*}\right)^{n}$ on the special fiber $X_{0}$ giving it the structure of a toric variety.

For the definition of a flat family (of schemes) see [Ha77, III.9]. In a flat family all the fibers $X_{t}=\pi^{-1}(t)$ have the same dimension, and more generally the same Hilbert polynomial ([Ha77, Theorem III.9.9 and Corollary III.9.10]). In fact the Hilbert polynomial being the same for all the fibers is equivalent to flatness. We note that flatness of a family $\pi: \mathfrak{X} \rightarrow \mathbb{C}$, in the case when $\mathfrak{X}$ is a variety, is a mild condition ([Ha77, Proposition III.9.7]).

We now describe the contents of this paper in more detail. The paper has two parts. In Part 1 we use a toric degeneration of $X$, satisfying some additional hypotheses explained below, to construct an integrable system on $X$. In Part 2 we show that this construction can be applied to the toric degenerations arising from the theory of Newton-Okounkov bodies (also called Okounkov bodies), as explained in [An13. As a consequence, we obtain integrable systems whose moment images are the corresponding Newton-Okounkov bodies.

First we present precise statements of the hypotheses and the result in our main theorem in Part 1 (Theorem A below). We require the toric degenerations to satisfy some additional properties as follows. Let $\pi: \mathfrak{X} \rightarrow \mathbb{C}$ be a toric degeneration of $X$ in the sense above and fix the isomorphism $\rho: X \times \mathbb{C}^{*} \rightarrow \pi^{-1}\left(\mathbb{C}^{*}\right)$. Note that $\mathbb{C}^{*}$ acts on $X \times \mathbb{C}^{*}$ by acting standardly on the second factor, and the isomorphism $\rho$ then induces a $\mathbb{C}^{*}$-action on $\mathfrak{X} \backslash X_{0}$. We assume the following:

(a) The family $\mathfrak{X}$ is smooth away from the singular locus of the zero fiber $X_{0}$. In particular, the projective variety $X$ is smooth.

(b) The family $\mathfrak{X}$ is embedded in $\mathbb{P}^{N} \times \mathbb{C}$ as an algebraic subvariety for some projective space $\mathbb{P}^{N}$ such that the following hold:

- the map $\pi$ is the restriction to $\mathfrak{X}$ of the standard projection $\mathbb{P}^{N} \times \mathbb{C} \rightarrow \mathbb{C}$ to the second factor, and

- the action of $\mathbb{T}=\left(\mathbb{C}^{*}\right)^{n}$ on $X_{0}$ extends to a linear action of $\mathbb{T}$ on $\mathbb{P}^{N} \times\{0\}$.

Now let $\omega$ be a fixed Kähler structure on $X$, the general fiber of $\mathfrak{X}$ (note $X$ is smooth by assumption (a) above). In our constructions, we also require that $\mathbb{P}^{N} \times \mathbb{C}$ (and hence $\mathfrak{X}$ ) is equipped with a Kähler form which is compatible with $\omega$ in a sense we now explain. Namely, let $\Omega$ be a Kähler form on $\mathbb{P}^{N}$ which is a constant multiple of a Fubini-Study Kähler form (see Remark 1.2) and equip $\mathbb{P}^{N} \times \mathbb{C}$ with the product Kähler structure given by $\Omega$ and the standard Kähler structure on $\mathbb{C}$. We denote by $\tilde{\omega}$ (respectively $\omega_{t}$ ) the restriction of this product form to the smooth locus of the family $\mathfrak{X}$ (respectively the fiber $X_{t}:=\pi^{-1}(t)$ ). For $t \neq 0$, denote by $\rho_{t}$ the restriction of the isomorphism $\rho: X \times \mathbb{C}^{*} \rightarrow \mathfrak{X} \backslash X_{0}$ to $X \times\{t\}$; this is an isomorphism of varieties between $X$ and the fiber $X_{t}$. Also let $T=\left(S^{1}\right)^{n}$ denote the compact torus, considered as a subgroup of $\mathbb{T}=\left(\mathbb{C}^{*}\right)^{n}$ in the standard fashion. With this notation in place we can state the following assumptions on our toric degenerations.

(c) The pull-back $\rho_{1}^{*}\left(\omega_{1}\right)$ to $X$ coincides with the original Kähler form $\omega$ on $X$.

(d) The Kähler form $\Omega$ on $\mathbb{P}^{N}$ is $T$-invariant; in particular, $\omega_{0}$ is a $T$-invariant Kähler form on the toric variety $X_{0}$. 
The following is the main result of Part 1 (Theorem 5.2).

Theorem (A). Let $X$ be a smooth $n$-dimensional projective variety and let $\omega$ be a Kähler structure on $X$. Suppose that there exists a toric degeneration $\pi: \mathfrak{X} \rightarrow \mathbb{C}$ of $X$, in the sense of Definition 2, satisfying the above conditions (a)-(d). Then:

(1) There exists a surjective continuous map $\phi: X \rightarrow X_{0}$ which is a symplectomorphism restricted to a dense open subset $U \subset X$.

(2) There exists a completely integrable system $\mu=\left(F_{1}, \ldots, F_{n}\right)$ on $(X, \omega)$, in the sense of Definition 1 above, such that its moment image $\Delta$ coincides with the moment image of $\left(X_{0}, \omega_{0}\right)$ (which is a polytope).

(3) Let $U \subset X$ be the open dense subset of $X$ from (1). Then the integrable system $\mu=\left(F_{1}, \ldots, F_{n}\right)$ generates a Hamiltonian torus action on $U$, and the inverse image $\mu^{-1}\left(\Delta^{\circ}\right)$ of the interior of $\Delta$ under the moment map $\mu: X \rightarrow \mathbb{R}^{n}$ of the integrable system lies in the open subset $U$.

We note that:

(i) For Theorem (A) part (1), we do not need to assume that $X_{0}$ is a toric variety and that the Kähler form $\omega_{0}$ is torus-invariant.

(ii) Theorem (A) still holds (with the same proof) if the special fiber $X_{0}$ is a union of toric varieties and hence possibly reducible as a variety. In this case the moment image will be a union of convex polytopes.

We now explain our main result in Part 2, where we apply Theorem A to the toric degenerations associated to valuations and Newton-Okounkov bodies. The theory of NewtonOkounkov bodies is newer than that of toric degenerations and integrable systems, and was initiated by Okounkov Ok96, Ok00 and further developed by Kaveh and Khovanskii KaKh08, KaKh-NO and also independently by Lazarsfeld and Mustata LaMu09. The notion of a Newton-Okounkov body associated to a projective variety far generalizes the notion of Newton polytope of a projective toric variety.

Let $X$ be a smooth $n$-dimensional projective variety equipped with a very ample line bundle $\mathcal{L}$ and let $R$ denote the corresponding homogeneous coordinate ring. Let $v: \mathbb{C}(X) \backslash\{0\} \rightarrow$ $\mathbb{Z}^{n}$ be a valuation with one-dimensional leaves (see Definition 7.1) on the space of rational functions $\mathbb{C}(X)$ on $X$, and let $S=S(R)$ denote the corresponding value semigroup in $\mathbb{N} \times \mathbb{Z}^{n}$ (see Equation (7.2)). We also let $\Delta=\Delta(R)$ denote the Newton-Okounkov body (also called Okounkov body) corresponding to $R$ and $v$ (Definition 7.7). Fix a Hermitian structure $H$ on $\mathcal{L}$ and let $\omega$ denote the associated Kähler structure on $X$, induced from the Kodaira embedding to $\mathbb{P}\left(H^{0}(X, \mathcal{L})^{*}\right)$ and the Fubini-Study form on $\mathbb{P}\left(H^{0}(X, \mathcal{L})^{*}\right)$ determined by $H$. With this notation in place we may state the main result of Part 2 (Theorem 10.1).

Theorem (B). Let $(X, \omega)$ and $S=S(R)$ be as above, and suppose in addition that the semigroup $S=S(R)$ is finitely generated.

(1) There exists an integrable system $\mu=\left(F_{1}, \ldots, F_{n}\right): X \rightarrow \mathbb{R}^{n}$ on $(X, \omega)$ in the sense of Definition 1. Moreover, the image of $\mu$ coincides with the Newton-Okounkov body $\Delta=\Delta(R)$.

(2) Let $U \subset X$ denote the open dense subset of $X$ mentioned in Definition 1 . Then the integrable system generates a torus action on $U$ and the inverse image under $\mu$ of the interior of the moment polytope $\Delta$ lies entirely in the open subset $U$.

Theorem (B) can be regarded as a generalization of the fact that the Newton polytope of a toric variety coincides with the image of its moment map, i.e. its moment polytope. 
In particular, Theorem B addresses a question posed to us by Julius Ross and by Steve Zelditch: does there exist, in general, a "reasonable" map from a (smooth) variety $X$ to its Newton-Okounkov body? At least under the technical assumption that the value semigroup $S=S(R)$ is finitely generated, our theorem suggests that the answer is yes, given by the map $\mu$ in Theorem B. Indeed, in this case we can do even better: we have a surjective continuous map $\phi$ from $X$ to the toric variety $X_{0}$ associated to the semigroup $S$, which is a symplectomorphism on a dense open subset. (The normalization of $X_{0}$ is the toric variety associated to the polytope $\Delta$.)

The present manuscript opens many doors. We now discuss a small selection of possible avenues for further investigation. Firstly, in future work we intend to further analyze (and in particular, make explicit computations for) the concrete examples mentioned in this paper (see Section 12), as well as the moduli spaces of flat connections on Riemann surfaces and Jeffrey-Weitsman integrable system (JeWe). Secondly, we intend to apply our results to provide explicit geometric interpretations of crystal bases for representations of connected reductive algebraic groups through the theory of geometric quantization and Bohr-Sommerfeld fibers (see e.g. Bu10) using methods similar to the work of Hamilton and Konno in HaKo11. Thirdly, it has been suggested to us by Milena Pabiniak and Yael Karshon that Theorem B can be applied to prove Biran's conjecture on the Gromov width of projective varieties (cf. KaTo05) for a large class of examples. Fourthly, in the classical case of the Gel'fand-Cetlin integrable system of Example 2 it is well-known that the action variables given by taking eigenvalues of upper-left $k \times k$ submatrices (which give continuous but non-smooth functions on $\operatorname{GL}(n, \mathbb{C}) / B$ ) can be "smoothed out" by instead taking the coefficients of the characteristic polynomials of the same upper-left submatrices. It would be interesting to better understand whether this phenomenon occurs for the action variables on $X$ obtained in this manuscript.

Finally, we discuss the issue of the technical hypothesis present in Theorem B above, namely, the finite generation of the semigroup $S=S(R)$. Firstly, in the light of Teissier's work Te99] which constructs toric degenerations even in the non-finitely-generated setting, we expect that the methods of this manuscript can be modified to prove statements similar to Theorem B even in the case when the value semigroup $S$ is not finitely generated. In general, given a projective variety $X$ and a valuation $v$, determining whether or not the corresponding semigroup $S$ is finitely generated is a subtle and tricky question. Indeed, it is not even known for an arbitrary $X$ whether one can always find a valuation $v$ with a corresponding value semigroup $S$ that is finitely generated. (However, it is known that such valuations exist in many interesting and important examples - cf. Section 12. In the papers of Anderson An13 and Anderson-Kuronya-Lozovanu AKL12, the authors give sufficient conditions on $X$ such that one can find a valuation $v$ with finitely generated semigroup $S$, but the relation between the choice of valuation and its corresponding value semigroup (and Newton-Okounkov body) is still not completely understood and there are many open questions.

We now briefly sketch the ideas of the proofs of our main results. The essential ingredient in the proof of Theorem A is the so-called "gradient-Hamiltonian vector field" (defined by Ruan [Ru99] and also used by Nishinou-Nohara-Ueno [NiNoUe10]) on $\mathfrak{X}$, where we think of $\mathfrak{X}$ as a Kähler space by embedding it into the product of an appropriate projective space with $\mathbb{C}$. The proof of Theorem B relies on a toric degeneration from $X$ to a toric variety $X_{0}$ (the normalization of which is the toric variety corresponding to the polytope $\Delta=\Delta(R)$ ), discussed in detail by Anderson An13. Let $\pi: \mathfrak{X} \rightarrow \mathbb{C}$ denote the flat family with special fiber $\pi^{-1}(0) \cong X_{0}$ and $\pi^{-1}(z)=X_{z} \cong X$ for $z \neq 0$. Since toric varieties are integrable 
systems (see Example 1 above), the essential idea is to "pull back" the integrable system on $X_{0}$ to one on $X$ using the gradient-Hamiltonian vector field. The main technicalities which must be overcome in order to make this idea rigorous is to appropriately deal with the singular points of $\mathfrak{X}$ and prove that the $f_{i}$ thus constructed may be continuously extended to all of $X$. It turns out that, in order to deal with this issue, we need a subtle generalization of the famous Łojasiewicz inequality. Finally, we note that since we use both algebro-geometric and differential-geometric techniques in our arguments, we work with both the Zariski and the classical (analytic) topologies on algebraic varieties. We have attempted to make clear in our statements which topology is meant.

Acknowledgements. As we were preparing this manuscript, we learned that Allen Knutson had roughly predicted our result in the MathOverflow discussion thread mentioned above. We thank Allen for encouraging us to work out these ideas carefully. We also thank Chris Manon and Dave Anderson for useful discussions. Finally, we thank the anonymous referees for useful comments which greatly improved the paper and for pointing out a short proof for Proposition 3.1 .

\section{Part 1. Integrable systems from toric degenerations}

\section{Preliminaries for Part 1}

We begin by recalling some background. Let $(M, \omega)$ be a symplectic manifold. A smooth function $H \in C^{\infty}(M)$, also called a Hamiltonian, defines a vector field $\xi_{H}$, called the Hamiltonian vector field (associated to $H$ ) by the equality

$$
\omega\left(\xi_{H}, \cdot\right)=d H(\cdot) .
$$

The Hamiltonian vector field defines a differential equation on $M$, the Hamiltonian system associated to $H$. The equation (1.1) also defines in turn a Poisson bracket on $C^{\infty}(M)$ by

$$
\{f, g\}:=\omega\left(\xi_{g}, \xi_{f}\right) \text {. }
$$

A function $f$ on $M$ is said to be a first integral of $H$ if $\{H, f\}=0$. A Hamiltonian system $(M, \omega, H)$ on a $2 n$-dimensional symplectic manifold is completely integrable (or integrable for short) if there exist $n=\frac{1}{2} \operatorname{dim}_{\mathbb{R}} M$ first integrals $H_{1}, H_{2}, \ldots, H_{n}$ which

- are functionally independent, i.e., there exists an open dense subset $U$ of $M$ such that, for all $x \in U$, the differentials $d H_{i}(x)$ are linearly independent,

- pairwise Poisson-commute, i.e., $\left\{H_{i}, H_{j}\right\}=0$ for all $i, j$.

By slight abuse of language one often refers to the collection $\left\{H_{1}, H_{2}, \ldots, H_{n}\right\}$ as an integrable system on $(M, \omega)$.

There are many interesting and well-studied examples of integrable systems on symplectic manifolds (cf. Au96 and references therein). However, as far as we are aware, little is known about methods for constructing interesting integrable systems on a general symplectic manifold $(M, \omega)$. Moreover, in many cases, a (smooth) set of action variables for an integrable system exists only on an open dense subset of $M$. A famous example is the Gel'fand-Cetlin system on coadjoint orbits of $U(n)$ described by Guillemin and Sternberg GuSt83. Here, the eigenvalues of the upper-left $k \times k$ submatrices give rise to action variables, but only on an open dense subset (because they are not smooth everywhere). The eigenvalues are, however, continuous everywhere. In general, it is also desirable to know that a set of smooth functions $\left\{H_{1}, H_{2}, \ldots, H_{\frac{1}{2} \operatorname{dim}_{\mathbb{R}} M}\right\}$ giving an integrable system (or, even better, action variables for an integrable system) on an open dense subset of $M$ actually extends continuously to all of $M$. 
The above discussion motivates the following definition.

Definition 1.1. Let $X$ be a complex variety of dimension $n$ (and hence of real dimension $2 n)$. Let $\omega$ be a symplectic form on the smooth locus of $X$. We call a collection of realvalued functions $\left\{F_{1}, \ldots, F_{n}\right\}$ on $X$ a completely integrable system (or an integrable system) on $X$ if the following are satisfied:

(a) $F_{1}, \ldots, F_{n}$ are continuous on all of $X$.

(b) There exists an open dense subset $U$ contained in the smooth locus of $X$ such that $F_{1}, \ldots, F_{n}$ are differentiable on $U$, and the differentials $d F_{1}, \ldots, d F_{n}$ are linearly independent on $U$.

(c) $F_{1}, \ldots, F_{n}$ pairwise Poisson-commute on $U$.

We call the map $\mu:=\left(F_{1}, \ldots, F_{n}\right): X \rightarrow \mathbb{R}^{n}$ the moment map of the integrable system.

Fix a Kähler form $\omega$ on a complex projective variety $X$. The main goal of the first part of this manuscript is to show that one can use a "good" toric degeneration of $X$ to construct an integrable system $\left\{F_{1}, \ldots, F_{n}\right\}$ on $(X, \omega)$ in the sense of Definition 1.1 .

An important class of completely integrable systems in symplectic geometry is given by taking components of the moment map of a compact symplectic toric manifold. (The analogous objects in the algebraic category are projective toric varieties.) Let $\mathbb{T}=\left(\mathbb{C}^{*}\right)^{n}$ denote an algebraic torus. Let $T=\left(S^{1}\right)^{n}$ denote the corresponding compact torus and let $V$ be a finite-dimensional $\mathbb{T}$-module. The action of $\mathbb{T}$ on $V$ induces an action on the projective space $\mathbb{P}(V)$. Suppose $H$ is a $T$-invariant Hermitian form on $V$. Then the corresponding Fubini-Study Kähler form $\Omega_{H}$ on $\mathbb{P}(V)$ is $T$-invariant. In fact, more is true: the $T$-action on $\left(\mathbb{P}(V), \Omega_{H}\right)$ is Hamiltonian, with moment map $\mu: \mathbb{P}(V) \rightarrow \operatorname{Lie}(T)^{*}$ given by

$$
\langle\mu(x), \xi\rangle=\frac{i}{2} \frac{H(\xi \cdot \tilde{x}, \tilde{x})}{H(\tilde{x}, \tilde{x})}
$$

where $\xi \in \operatorname{Lie}(T)$ and $\tilde{x} \in V$ represents the point $x \in \mathbb{P}(V)$.

Let $\mathcal{A}=\left\{\alpha_{0}, \ldots, \alpha_{r}\right\} \subset \mathbb{Z}^{n}$ be a finite set of integral points. Let $\mathbb{T}$ act linearly on $V=\mathbb{C}^{r+1}$ via weights $\alpha_{0}, \ldots, \alpha_{r}$, that is:

$$
x \cdot\left(z_{0}, \cdots, z_{r}\right)=\left(x^{\alpha_{0}} z_{0}, \cdots, x^{\alpha_{r}} z_{r}\right),
$$

where $x \in \mathbb{T}$ and we use shorthand notation $x^{\alpha}=x_{1}^{a_{1}} \cdots x_{n}^{a_{n}}$ for $\alpha=\left(a_{1}, \ldots, a_{n}\right), x=$ $\left(x_{1}, \ldots, x_{n}\right)$. We assume that $\mathcal{A}$ is large enough so that the induced action of $\mathbb{T}$ on $\mathbb{P}(V)$ is faithful. Then the orbit of a generic point, e.g. $(1: \cdots: 1)$ is isomorphic to $\mathbb{T}$ itself. Let $X_{\mathcal{A}}$ denote the closure of the orbit of $(1: \cdots: 1)$. Then $X$ is a (not necessarily smooth or normal) projective toric variety. Take the Fubini-Study Kähler form $\Omega$ on $\mathbb{P}(V)$ with respect to the standard Hermitian product on $V=\mathbb{C}^{r+1}$. It is invariant under the action of the real torus $T=\left(S^{1}\right)^{n}$. Equip $X_{\mathcal{A}}$ (more precisely the smooth locus of $X_{\mathcal{A}}$ ) with the Kähler structure induced from $\mathbb{P}(V)$. The moment map $\mu$ for the projective space $\mathbb{P}(V)$ restricts to a moment map $\mu_{\mathcal{A}}: X_{\mathcal{A}} \rightarrow \operatorname{Lie}(T)^{*} \cong \mathbb{R}^{n}$ for $X_{\mathcal{A}}$. The image of $\mu_{\mathcal{A}}$ is the convex hull $\Delta=\operatorname{conv}(\mathcal{A})$. Let $\left\{H_{1}, \ldots, H_{n}\right\}$ denote the components of $\mu_{\mathcal{A}}$ with respect to some choice of a basis for $\operatorname{Lie}(T)$. Since $X_{\mathcal{A}}$ is a toric variety, i.e. has a dense open orbit isomorphic to $\mathbb{T}$ itself, we immediately conclude:

- The collection $\left\{H_{1}, \ldots, H_{n}\right\}$ is an integrable system on $X_{\mathcal{A}}$, in the sense of Definition 1.1

- The image of the moment map $\mu_{\mathcal{A}}=\left(H_{1}, \ldots, H_{n}\right)$ is the rational polytope $\Delta=$ $\operatorname{conv}(\mathcal{A})$. 
- By construction, the Hamiltonians $H_{1}, \ldots, H_{n}$ generate a (Hamiltonian) torus action on $X_{\mathcal{A}}$.

Remark 1.2. If we replace the Kähler form $\Omega$ by $c \Omega$ for some non-zero real number $c$, then the moment map and the moment image for the $T$-action are replaced with $c \mu$ and $c \Delta$ respectively. In particular, the moment image may no longer be an integral polytope. This situation arises later in Part 2 where we deal with toric degenerations to projective toric varieties associated to polytopes with rational vertices. In this case the Kähler form on the toric variety coming from the polytope is the restriction of a constant multiple of the Fubini-Study form.

\section{Gradient-Hamiltonian Flow}

Let $\mathfrak{X}$ be an algebraic variety equipped with a Kähler structure $\tilde{\omega}$ on its smooth locus $\mathfrak{X}_{\text {smooth }}$. Let $\pi: \mathfrak{X} \rightarrow \mathbb{C}$ be a morphism (i.e. an algebraic map). We let $X_{t}$ denote the fiber $\pi^{-1}(t)$ and $\omega_{t}$ denote the restriction of $\tilde{\omega}$ to the smooth points of $X_{t} \cap \mathfrak{X}_{\text {smooth }}$. Following Ruan Ru99, we now define the gradient-Hamiltonian vector field corresponding to $\pi$ on $\mathfrak{X}_{\text {smooth }}$ as follows. Let $\nabla(\operatorname{Re}(\pi))$ denote the gradient vector field on $\mathfrak{X}_{\text {smooth }}$ associated to the real part $\operatorname{Re}(\pi)$, with respect to the Kähler metric. Since $\tilde{\omega}$ is Kähler and $\pi$ is holomorphic, the Cauchy-Riemann equations imply that $\nabla(\operatorname{Re}(\pi))$ is related to the Hamiltonian vector field $\xi_{\operatorname{Im}(\pi)}$ of the imaginary part $\operatorname{Im}(\pi)$ with respect to the Kähler (symplectic) form $\tilde{\omega}$ by

$$
\nabla(\operatorname{Re}(\pi))=-\xi_{\operatorname{Im}(\pi)} .
$$

Let $Z \subset \mathfrak{X}$ denote the closed set which is the union of the singular locus of $\mathfrak{X}$ and the critical set of $\operatorname{Re}(\pi)$, i.e. the set on which $\nabla(\operatorname{Re}(\pi))=0$. The gradient-Hamiltonian vector field $V_{\pi}$, which is defined only on the open set $\mathfrak{X} \backslash Z$, is by definition

$$
V_{\pi}:=-\frac{\nabla(\operatorname{Re}(\pi))}{\|\nabla(\operatorname{Re}(\pi))\|^{2}} .
$$

Where defined, $V_{\pi}$ is smooth. Moreover, from 2.2 it follows that

$$
V_{\pi}(\operatorname{Re}(\pi))=-1 .
$$

For $t \in \mathbb{R}_{>0}$ let $\phi_{t}$ denote the time- $t$ flow corresponding to $V_{\pi}$. Since $V_{\pi}$ may not be complete, $\phi_{t}$ for a given $t$ is not necessarily defined on all of $\mathfrak{X} \backslash Z$. We record the following facts.

Proposition 2.1. Let the notation be as introduced above.

(a) Suppose $s, t \in \mathbb{R}$ with $s \geq t>0$. Where defined, the flow $\phi_{t}$ takes $X_{s} \cap(\mathfrak{X} \backslash Z)$ to $X_{s-t}$. In particular, where defined, $\phi_{t}$ takes a point $x \in X_{t}$ to a point in the fiber $X_{0}$. Moreover, if the $\mathfrak{X}$ is smooth away from $X_{0}$ and there are no critical points of $\pi$ in $\pi^{-1}([s, s-t])$, then $\phi_{t}$ is defined everywhere on $X_{s}$.

(b) Where defined, the flow $\phi_{t}$ preserves the symplectic structures, i.e., if $x \in X_{z} \cap(\mathfrak{X} \backslash Z)$ is a point where $\phi_{t}(x)$ is defined, then $\phi_{t}^{*}\left(\omega_{z-t}\right)_{\phi_{t}(x)}=\left(\omega_{z}\right)_{x}$.

Recall that a smooth point $x \in \mathfrak{X}$ is a critical point of $\pi$ if $d \pi(x)=0$. A scalar $z \in \mathbb{C}$ is a critical value if $X_{z}$ contains at least one critical point. Note that by the Cauchy-Riemann relations $d \pi=0$ if and only if $d(\operatorname{Re}(\pi))=0$, which in turn holds if and only if $\nabla(\operatorname{Re}(\pi))=0$. The next lemma is immediate from generic smoothness [Ha77, Corollary III.10.7]. 
Lemma 2.2. Let $\pi: \mathfrak{X} \rightarrow \mathbb{C}$ be a morphism. Then the set of critical values of $\pi: \mathfrak{X}_{\text {smooth }} \rightarrow$ $\mathbb{C}$ is a finite set. In particular there exists $\epsilon>0$ such that there is no critical value in the interval $(0, \epsilon]$.

Remark 2.3. By definition the families we consider in Section 5 (namely toric degenerations) are trivial over $\mathbb{C}^{*}$. Moreover we assume that the generic fiber is smooth. In this case the only possible critical value is 0 and Lemma 2.2 is not needed.

Next we recall the following fundamental theorem on the solutions of ordinary differential equations (see for example [HuLi05, Theorem 1.7.1]):

Theorem 2.4. Let $V$ be a continuously differentiable vector field defined in an open subset $U$ of a Euclidean space. Then for any $x_{0} \in U$, the flow $\phi_{t}$ of $V$ is defined at $x_{0}$ for $0 \leq t<b$ where we have the following possibilities:

(1) $b=\infty$ or

(2) $b<\infty$ and $\phi_{t}\left(x_{0}\right)$ is unbounded as $t \rightarrow b^{-}$, or

(3) $b<\infty$ and $\phi_{t}\left(x_{0}\right)$ approaches the boundary of $U$ as $t \rightarrow b^{-}$.

Let $U_{0} \subset X_{0}$ denote the open set $X_{0} \backslash\left(X_{0} \cap Z\right)$. Then the gradient-Hamiltonian vector field is defined at all $x_{0} \in U_{0}$. Note that, a priori, the set $U_{0}$ may be empty; however, in the next section (Section 3 ) we prove that under some assumptions on the family $\mathfrak{X}$, the set $U_{0}$ is in fact dense in $X_{0}$.

Lemma 2.5. Let the notation be as above. Assume that the family $\mathfrak{X}$ is smooth away from the fiber $X_{0}$. Further assume that the map $\pi: \mathfrak{X} \rightarrow \mathbb{C}$ is proper with respect to the classical topologies on $\mathfrak{X}$ and $\mathbb{C}$. Let $\epsilon>0$ be such that there are no critical values in $(0, \epsilon] \subset \mathbb{C}$. Then the flow $\phi_{-\epsilon}$ is defined for all $x_{0} \in U_{0}$. In particular, if there are no critical values in $(0,+\infty)$ then $\phi_{-t}$ is defined for all $x \in U_{0}$ and all $t>0$.

Proof. Let $x_{0} \in U_{0}$. From the theory of ODEs we know there exists $b>0$ such that the flow $\phi_{-t}\left(x_{0}\right)$ is defined for $t \in[0, b)$. If $b \geq \epsilon$ there is nothing to prove, so suppose $b<\epsilon$. From Proposition 2.1 (a) we also know that $\phi_{-t}\left(x_{0}\right) \in X_{t}$ for each such $t$. Thus the flow defines a continuous map from $[0, b)$ to $\pi^{-1}([0, b])$ by $t \mapsto \phi_{-t}\left(x_{0}\right)$. Since $\pi$ is proper, the inverse image $\pi^{-1}([0, b])$ is a compact set, so there exists a point $x_{b} \in X_{b}$ such that $\phi_{-t}\left(x_{0}\right) \rightarrow x_{b}$ as $t \rightarrow b^{-}$. By assumption on the family $\mathfrak{X}$, the fiber $X_{b}$ lies in the smooth locus of $\mathfrak{X}$. Moreover by the assumption on $\epsilon$ we also know that $X_{b}$ contains no critical points of $\pi$. Thus the limit point $x_{b}$ is contained in $\mathfrak{X} \backslash Z$ and so by Theorem 2.4 we conclude the flow is defined at $t=b$ and takes the value $\phi_{-b}\left(x_{0}\right)=x_{b}$. Again by the theory of ODEs this implies the flow is defined not just on $[0, b)$ but on $\left[0, b^{\prime}\right)$ for some $b^{\prime}>b$. Repeating the above argument with $b^{\prime}$ in place of $b$ as necessary shows that the flow is in fact defined also at $t=\epsilon$, as desired.

\section{Critical points of the family}

In this section we show that under mild assumptions on the family $\mathfrak{X}$, the set of critical points of $\pi$ intersected with $X_{0}$ lies in the singular locus of $X_{0}$. It follows that the set $U_{0} \subseteq X_{0}$ discussed in the previous section is open and dense in $X_{0}$ (Corollary 3.3).

By a flat family of varieties over $\mathbb{C}$ we mean a flat morphism $\pi: \mathfrak{X} \rightarrow \mathbb{C}$ on a quasiprojective variety $\mathfrak{X}$ such that the fibers are reduced as schemes. Since $\pi$ is a flat morphism all the fibers have the same dimension $n=\operatorname{dim}(\mathfrak{X})-1$. Before stating the next result we note that without loss of generality we can always assume that the family $\mathfrak{X}$ is embedded in $\mathbb{P}^{N} \times \mathbb{C}$ for some projective space $\mathbb{P}^{N}$ and that the map $\pi$ is the restriction of the projection 
$\mathbb{P}^{N} \times \mathbb{C} \rightarrow \mathbb{C}$ on the second factor. To see this, let $i: \mathfrak{X} \hookrightarrow \mathbb{P}^{N}$ be an embedding of the quasi-projective variety $\mathfrak{X}$ in a projective space $\mathbb{P}^{N}$. Then the map $\tilde{i}: \mathfrak{X} \rightarrow \mathbb{P}^{N} \times \mathbb{C}$ given by $\tilde{i}(x)=(i(x), \pi(x))$ is also an embedding and $\pi=p_{2} \circ \tilde{i}$ where $p_{2}$ is the projection on the second factor, as claimed.

Proposition 3.1. Let $\pi: \mathfrak{X} \rightarrow \mathbb{C}$ be a flat family of varieties with $\operatorname{dim}(\mathfrak{X})=n+1$. Consider a fiber $X_{z}=\pi^{-1}(z)$. If $p \in X_{z}$ is a smooth point of $X_{z}$ then it is a smooth point of $\mathfrak{X}$ as well.

Proof. A point $p$ is a smooth point of $X_{z}$ if and only if $\operatorname{dim}\left(T_{p} X_{z}\right)=\operatorname{dim}\left(X_{z}\right)$ which in turn is equal to $\operatorname{dim}(\mathfrak{X})-1$ because of the flatness of the family. Since $X_{z}$ is a Cartier divisor in $\mathfrak{X}$ we know $\operatorname{dim}\left(T_{p} \mathfrak{X}\right)-1 \leq \operatorname{dim}\left(T_{p} X_{z}\right)$. So $\operatorname{dim}\left(T_{p} \mathfrak{X}\right) \leq \operatorname{dim}(\mathfrak{X})$, i.e. $p$ is a smooth point of $\mathfrak{X}$.

We now apply this to our setting in Section 2 Recall that $Z \subset \mathfrak{X}$ denotes the closed set which is the union of the singular locus of $\mathfrak{X}$ and the critical set of $\pi$, and $U_{0}$ denotes the (Zariski-open) subset $X_{0} \backslash\left(X_{0} \cap Z\right)$.

We state the following simple fact whose proof is immediate from the definitions.

Lemma 3.2. Let $\mathfrak{X}$ be an algebraic variety and $\pi: \mathfrak{X} \rightarrow \mathbb{C}$ a morphism. Suppose a fiber $X_{z}$ is reduced as a scheme. Take a point $p \in X_{z}$ such that $\mathfrak{X}$ is smooth at $p$. If $p$ is a critical point of $\pi$, i.e. $d \pi(p)=0$, then $p$ is a singular point of $X_{z}$.

The following is a simple consequence of Proposition 3.1 and the above lemma applied to the fiber $X_{0}$.

Corollary 3.3. Let $\pi: \mathfrak{X} \rightarrow \mathbb{C}$ be a flat family of varieties. Consider the fiber $X_{0}$. Then $\pi$ does not have any critical points on the smooth locus of $X_{0}$. It follows that $U_{0}$ is precisely the smooth locus of $X_{0}$ and in particular $U_{0}$ is dense in $X_{0}$.

Proof. Take $p \in X_{0}$ which is a smooth point for $X_{0}$. By Proposition 3.1 $p$ is a smooth point of $\mathfrak{X}$ as well. Now $p$ cannot be a critical point of $\pi$ because otherwise by Lemma 3.2 it should be a singular point of $X_{0}$ which is a contradiction. This proves the claim.

For the next corollary we need to equip the family $\mathfrak{X}$ with a Kähler structure. Suppose $\Omega$ is a Fubini-Study Kähler form on $\mathbb{P}^{N}$ and suppose $\mathbb{P}^{N} \times \mathbb{C}$ is equipped with the product Kähler structure $\Omega \times\left(\frac{i}{2} d z \wedge d \bar{z}\right)$ where the second factor is the standard Kähler structure on $\mathbb{C}$ (here $z$ is the coordinate on $\mathbb{C}$ ). We assume that (the smooth locus of) $\mathfrak{X}$ is equipped with the Kähler structure obtained by restricting this product structure to $\mathfrak{X}$.

Corollary 3.4. With notation and assumptions as in the discussion above and Corollary 3.3. let $\epsilon>0$ be as in Lemma 2.5 i.e. there are no critical values of $\pi$ in $(0, \epsilon]$. Then the image $U_{\epsilon}:=\phi_{-\epsilon}\left(U_{0}\right)$ of $U_{0}$ under the flow $\phi_{-\epsilon}$ is an open dense subset of $X_{\epsilon}$.

Proof. The flow $\phi_{-\epsilon}$ is a local diffeomorphism. Since $\phi_{-\epsilon}$ sends $U_{0}$ to $X_{\epsilon}$, the image $U_{\epsilon}:=$ $\phi_{-\epsilon}\left(U_{0}\right)$ is an open subset of $X_{\epsilon}$. It remains to show that $U_{\epsilon}$ is dense in $X_{\epsilon}$. To this end, recall that by Proposition 2.1 we know $\phi_{-\epsilon}^{*}\left(\omega_{\epsilon}\right)=\omega_{0}$. Thus

$$
\int_{X_{0}} \omega_{0}^{n}=\int_{U_{0}} \omega_{0}^{n}=\int_{U_{0}}\left(\phi_{-\epsilon}^{*}\left(\omega_{\epsilon}\right)\right)^{n}=\int_{U_{\epsilon}} \omega_{\epsilon}^{n}
$$

where the first equality uses Corollary 3.3 . By assumption, $\omega_{0}$ and $\omega_{\epsilon}$ are restrictions of the Kähler form $\Omega$ on $\mathbb{P}^{N}$. Furthermore, by [Mu76, Section $\left.5 \mathrm{C}\right]$, the symplectic volumes $\int_{X_{0}} \omega_{0}^{n}$ 
and $\int_{X_{\epsilon}} \omega_{\epsilon}^{n}$ are equal (up to a normalization factor, which is in fact $n !$ ) to the degrees of $X_{0}$ and $X_{\epsilon}$ regarded as subvarieties of $\mathbb{P}^{N}$. Since the family $\mathfrak{X}$ is flat, the degrees of $X_{0}$ and $X_{\epsilon}$ are equal (see [Ha77, Theorem III.9.9]) and thus

$$
\int_{X_{0}} \omega_{0}^{n}=\int_{X_{\epsilon}} \omega_{\epsilon}^{n}
$$

Equations (3.1) and 3.2 together imply that $\int_{U_{\epsilon}} \omega_{\epsilon}^{n}=\int_{X_{\epsilon}} \omega_{\epsilon}^{n}$. Thus $X_{\epsilon} \backslash U_{\epsilon}$ has empty interior and $U_{\epsilon}$ is dense in $X_{\epsilon}$ as desired.

\section{Continuity of the gradient-Hamiltonian Flow}

The main result of this (technical) section is Theorem 4.1, the proof of which depends on a subtle generalization of the famous "Lojasiewicz gradient inequality". The point of the theorem is to prove that for a small enough parameter $\epsilon>0$, the flow $\phi_{\epsilon}$ of the gradientHamiltonian vector field on $\mathfrak{X}$, which is a priori not defined at all points of $X_{\epsilon}$, can be extended to a continuous function on all of $X_{\epsilon}$. (The continuity is with respect to the classical topology.) We refer the reader to the paper of Lerman [Lerman for background, where we learned about gradient flows in the context of symplectic geometry.

In what follows we continue to place the hypotheses on the family $\mathfrak{X}$ and its Kähler structure from Sections 2 and 3 Specifically, we assume that $\pi: \mathfrak{X} \rightarrow \mathbb{C}$ is a flat family of irreducible varieties where $\mathfrak{X} \subset \mathbb{P}^{N} \times \mathbb{C}$ as an algebraic subvariety and $\pi$ is the restriction of the projection to the second factor. We also assume that $\mathfrak{X}$ is smooth away from the singular locus of $X_{0}$. We fix a Kähler form $\tilde{\omega}$ on $\mathfrak{X}$ which is the restriction of a product Kähler form on $\mathbb{P}^{N} \times \mathbb{C}$ of the form $\Omega \times\left(\frac{i}{2} d z \wedge d \bar{z}\right)$ where $\Omega$ is a Fubini-Study Kähler structure on $\mathbb{P}^{N}$. Recall that $\phi_{t}$ is the flow associated to the gradient-Hamiltonian vector field defined in Section 2 and $U_{0}$ is the open set $X_{0} \backslash\left(X_{0} \cap Z\right)$ where $Z$ is the closed set consisting of the union of the singular locus of $\mathfrak{X}$ and the critical set of $\pi$. Also recall that by Lemma 2.5 and Corollary 3.4 we know there exists an $\epsilon>0$ such that $U_{\epsilon}:=\phi_{-\epsilon}\left(U_{0}\right)$ is open and dense in $X_{\epsilon}$.

Theorem 4.1. With the notation and assumptions as above, let $\epsilon>0$ satisfy the condition of Lemma 2.5, i.e. there are no critical values in the interval $(0, \epsilon]$. Then the flow $\phi_{\epsilon}$ of the gradient-Hamiltonian vector field associated to $f:=\operatorname{Re}(\pi)$, a priori defined only on the (open and dense) subset $U_{\epsilon} \subseteq X_{\epsilon}$, extends to a continuous (with respect to the classical topology) function defined on all of $X_{\epsilon}$, with image in $X_{0}$.

Remark 4.2. In general, the flow with respect to the gradient vector field of a smooth function $f$ on a Riemannian manifold $X$ does not give rise to a continuous function between the level sets of $f$. The reason that we can continuously extend our flow $\phi_{\epsilon}$ is that we work in an algebraic (and hence real analytic) setting.

The proof of Theorem 4.1 is based on the famous "Lojasiewicz gradient inequality" for analytic functions (cf. [Lo84 and KuMoPa00, pages 763 and 765]):

Theorem 4.3. Let $f$ be a real-valued analytic function defined on some open subset $W \subset$ $\mathbb{R}^{m}$. Then for any $x \in W$ there exists an open neighborhood $U_{x}$ of $x$ and constants $c_{x}>0$ and $0<\alpha_{x}<1$ such that for all $y \in U_{x}$ :

$$
\|\nabla f(y)\| \geq c_{x}|f(y)-f(x)|^{\alpha_{x}}
$$

In fact, since $X_{0}$ may not be smooth, we will need the following generalization of Theorem 4.3 due to Kurdyka and Parusinski [Ku94, Proposition 1]. 
Theorem 4.4. Let $Y$ be a (possibly singular) algebraic subset of $\mathbb{R}^{m}$. Let $f: \mathbb{R}^{m} \rightarrow \mathbb{R}$ be a semi-algebraic function. Then for any $x \in Y$ (not necessarily a smooth point) there is an open neighborhood $U_{x} \subset Y$ (in the classical topology) and constants $c_{x}>0$ and $0<\alpha_{x}<1$ such that for any smooth point $y \in U_{x}$ we have:

$$
\|\nabla f(y)\| \geq c_{x}|f(y)-f(x)|^{\alpha_{x}}
$$

where $\nabla f$ denotes the gradient of $f_{\mid Y}$ with respect to the induced metric on the smooth locus of $Y$.

Remark 4.5. Since any Riemannian metric on a relatively compact subset of $\mathbb{R}^{n}$ (i.e. a subset with compact closure) induces a norm which is equivalent ${ }^{1}$ to the standard Euclidean norm, the inequalities 4.1 and 4.2 hold for an arbitrary Riemannian metric on $\mathbb{R}^{m}$ with the same exponent $\alpha_{x}$ and possibly different constant $c_{x}$.

Remark 4.6. In Ku94 the more general case of subanalytic functions and sets is addressed. For our purposes, the algebraic/semi-algebraic case stated above suffices.

Proof of Theorem 4.1. Let $f:=\operatorname{Re}(\pi)$. For $x \in X_{0}$, let $U_{x}, c_{x}>0$ and $0<\alpha_{x}<1$ be as in Theorem 4.4 (applied to the family $\mathfrak{X}$ ). Since $X_{0}$ is compact, it can be covered with finitely many such open sets $U_{x_{1}}, \ldots, U_{x_{s}}$. Let

$$
\begin{gathered}
U:=\left(\bigcup_{i=1}^{s} U_{x_{i}}\right) \cap\{y \in \mathfrak{X}|| f(y) \mid<\epsilon\} \subseteq \mathfrak{X} \\
c:=\min \left\{c_{x_{1}}, \ldots, c_{x_{s}}\right\}, \\
\alpha:=\max \left\{\alpha_{1}, \ldots, \alpha_{s}\right\} .
\end{gathered}
$$

Clearly $X_{0} \subset U$. Moreover, from 4.2 it follows that for any point $y \in U$ we have:

$$
\|\nabla f(y)\| \geq c|f(y)|^{\alpha} .
$$

Next we claim that there exists $\rho>0$ such that $X_{t} \subset U$ for all $0<t<\rho$. To see this, suppose for a contradiction that there exists a sequence $\left(x_{i}\right)_{i \in \mathbb{N}} \in \mathfrak{X}$ such that for all $i$ we have $x_{i} \notin U, 0<\pi\left(x_{i}\right)<1$, and $\lim _{i \rightarrow \infty} \pi\left(x_{i}\right)=0$. Since $\pi$ is proper, the set $\pi^{-1}([0,1]) \subseteq \mathfrak{X}$ is compact, from which it follows that the sequence $\left(x_{i}\right)$ has a limit point $x \in \mathfrak{X}$. Since $x_{i} \notin U$ for all $i$ and $U$ is open, we know $x \notin U$. On the other hand, by continuity $\pi(x)=0$ and hence $x \in X_{0} \subset U$, contradiction.

Now let $\rho>0$ be as above. From $\sqrt{4.3}$ we conclude that for any $0<t<\rho$ and $y \in X_{t}$ we have:

$$
\|\left.\nabla f(y)\right|^{-1} \leq(1 / c)|f(y)|^{-\alpha} .
$$

Note that $f(y)=\operatorname{Re}(t)=t$ is nonzero and hence division by $f(y)$ is allowed.

Also for any $\epsilon-\rho<t<\epsilon$ and for any $y \in X_{\epsilon}$ we have that $\phi_{t}(y)$ is defined (by an argument similar to the proof of Lemma 2.5 and that

$$
\phi_{t}(y) \in X_{\epsilon-t} \subset U \text {. }
$$

Let $x \in X_{\epsilon}$. Then for any $t_{0}, t_{1}$ with $\epsilon-\rho<t_{0}<t_{1}<\epsilon$, we have

$$
\left\|\phi_{t_{1}}(x)-\phi_{t_{0}}(x)\right\|=\left\|\int_{t_{0}}^{t_{1}} \frac{d}{d t} \phi_{t}(x) d t\right\|
$$

\footnotetext{
${ }^{1}$ We say that two norms $|\cdot|_{1}$ and $|\cdot|_{2}$ are equivalent if there exist constants $c, C>0$ such that $c|\cdot|_{1}<$ $|\cdot|_{2}<C|\cdot|_{1}$.
} 


$$
\begin{aligned}
& =\left\|\int_{t_{0}}^{t_{1}} V\left(\phi_{t}(x)\right) d t\right\| \\
& \leq \int_{t_{0}}^{t_{1}}\left\|V\left(\phi_{t}(x)\right)\right\| d t
\end{aligned}
$$

In addition, by 4.4 we have

$$
\left\|\phi_{t_{1}}(x)-\phi_{t_{0}}(x)\right\| \leq \int_{t_{0}}^{t_{1}}(1 / c)\left|f\left(\phi_{t}(x)\right)\right|^{-\alpha} d t
$$

and since $x \in X_{\epsilon}$, by Proposition 2.1 we have

$$
f\left(\phi_{t}(x)\right)=\epsilon-t .
$$

Putting this together we obtain

$$
\begin{aligned}
\left\|\phi_{t_{1}}(x)-\phi_{t_{0}}(x)\right\| & \leq \frac{1}{c} \int_{t_{0}}^{t_{1}}(\epsilon-t)^{-\alpha} d t \\
& \leq \frac{1}{c(1-\alpha)}\left(\left(\epsilon-t_{1}\right)^{1-\alpha}-\left(\epsilon-t_{0}\right)^{1-\alpha}\right) .
\end{aligned}
$$

Since $1-\alpha>0$ by assumption, we know $\lim _{t \rightarrow \epsilon}(\epsilon-t)^{1-\alpha}=0$. Therefore for any $\varepsilon^{\prime}>0$, there exists $\delta>0$ such that for any $x \in X_{1}$ and any $t_{0}, t_{1}$ with $1-\delta<t_{0}<t_{1}<1$, we have

$$
\left\|\phi_{t_{1}}(x)-\phi_{t_{0}}(x)\right\| \leq \varepsilon^{\prime} \text {. }
$$

It follows that for any $x \in X_{\epsilon}, \lim _{t \rightarrow \epsilon} \phi_{t}(x)$ exists. Let $\phi(x)$ denote this limit. By continuity, if the original flow $\phi_{\epsilon}$ was already defined at $x$, then $\phi_{\epsilon}(x)=\phi(x)$. Thus the map $\phi$ is an extension of $\phi_{\epsilon}$ to all of $X_{\epsilon}$, and by construction takes $X_{\epsilon}$ to $X_{0}$.

It remains to show that $\phi: X_{\epsilon} \rightarrow X_{0}$ is continuous. Let $x \in X_{\epsilon}$. We need to show that for any $\varepsilon^{\prime \prime}>0$ there exists $\delta>0$ such that if $y \in X_{\epsilon}$ and $\|y-x\|<\delta$ then $\|\phi(y)-\phi(x)\|<\varepsilon^{\prime \prime}$. By the above, we can choose $t \in \mathbb{R}$ with $0<t<\epsilon$ such that for any $y \in X_{\epsilon}$ we have

$$
\left\|\phi_{t}(y)-\phi(y)\right\|<\varepsilon^{\prime \prime} / 3 \text {. }
$$

Since the map $y \mapsto \phi_{t}(y)$ is continuous, we can find $\delta>0$ such that for any $y \in X_{\epsilon}$, if $\|x-y\|<\delta$ then $\left\|\phi_{t}(x)-\phi_{t}(y)\right\|<\varepsilon^{\prime \prime} / 3$. Now, if $\|x-y\|<\delta$, we have:

$$
\begin{aligned}
\|\phi(x)-\phi(y)\| & \leq\left\|\phi(x)-\phi_{t}(x)\right\|+\left\|\phi_{t}(x)-\phi_{t}(y)\right\|+\left\|\phi_{t}(y)-\phi(y)\right\| \\
& \leq \varepsilon^{\prime \prime} / 3+\varepsilon^{\prime \prime} / 3+\varepsilon^{\prime \prime} / 3 \\
& \leq \varepsilon^{\prime \prime}
\end{aligned}
$$

as desired.

\section{Construction of An Integrable System From A toric Degeneration}

With these preliminaries in place, we can now prove the main result of Part 1 of this manuscript, which constructs an integrable system in the sense of Definition 1.1 from a toric degeneration satisfying certain properties (described below).

We first recall the definition of a toric degeneration. Let $X$ be an $n$-dimensional projective variety. Let $\pi: \mathfrak{X} \rightarrow \mathbb{C}$ be a family of varieties.

Definition 5.1. We call $\pi: \mathfrak{X} \rightarrow \mathbb{C}$ a toric degeneration of $X$ if the following hold:

(1) $\pi: \mathfrak{X} \rightarrow \mathbb{C}$ is a flat family of irreducible varieties. In particular, each fiber $X_{t}:=$ $\pi^{-1}(t)$ is reduced regarded as a scheme. 
(2) The family $\mathfrak{X}$ is trivial over $\mathbb{C}^{*}$, i.e. there is a fiber-preserving isomorphism of varieties $\rho: X \times \mathbb{C}^{*} \rightarrow \mathfrak{X} \backslash X_{0}$. In particular, for any $t \in \mathbb{C}^{*}$, the restriction $\rho_{t}$ of $\rho$ to $X \times\{t\}$ yields an isomorphism of the varieties $X \cong X \times\{t\}$ and the fiber $X_{t}$.

(3) The fiber $X_{0}$ is a toric variety with respect to an action of $\mathbb{T}=\left(\mathbb{C}^{*}\right)^{n}$.

For the rest of our discussion we fix once and for all the isomorphism $\rho: X \times \mathbb{C}^{*} \rightarrow \mathfrak{X} \backslash X_{0}$ in item (2) above. Consider the diagonal action of $\mathbb{C}^{*}$ on $X \times \mathbb{C}^{*}$, where $\mathbb{C}^{*}$ acts trivially on the first factor and standardly on the second factor. The identification $\rho$ then induces a corresponding $\mathbb{C}^{*}$-action on $\mathfrak{X} \backslash X_{0}$ which preserves the fibers of $\pi$.

Next we state some hypotheses, listed in items (a)-(d) below, which we place on our toric degenerations. These hypotheses are natural in the sense that many of the known examples satisfy them (cf. Section 12 and Remark 5.4 below). For the following, suppose that the projective variety $X$ is equipped with a Kähler form $\omega$; the integrable system constructed in Theorem 5.2 is with respect to this $\omega$.

Let $\pi: \mathfrak{X} \rightarrow \mathbb{C}$ be a toric degeneration of $X$. We assume that:

(a) The family $\mathfrak{X}$ is smooth away from the singular locus of the zero fiber $X_{0}$.

(b) The family $\mathfrak{X}$ is embedded in $\mathbb{P}^{N} \times \mathbb{C}$ as an algebraic subvariety, for some projective space $\mathbb{P}^{N}$, such that:

- the map $\pi$ is the restriction to $\mathfrak{X}$ of the usual projection of $\mathbb{P}^{N} \times \mathbb{C}$ to its second factor, and

- the action of $\mathbb{T}$ on $X_{0}$ extends to a linear action on $\mathbb{P}^{N}$.

We also place some additional hypotheses on the Kähler form $\omega$ on $X$ as follows. Suppose $\Omega$ is a constant multiple of a Fubini-Study Kähler form on $\mathbb{P}^{N}$. Let $z$ denote the complex variable on $\mathbb{C}$ so that $\omega_{\text {std }}=\frac{i}{2} d z \wedge d \bar{z}$ is the standard Kähler form on $\mathbb{C}$. We equip $\mathbb{P}^{N} \times \mathbb{C}$ with the product Kähler structure $\Omega \times \omega_{\text {std }}$. Assuming that $\mathfrak{X}$ satisfies the conditions (a) and (b) above, so in particular $\mathfrak{X}$ is embedded in $\mathbb{P}^{N} \times \mathbb{C}$, let $\tilde{\omega}$ (respectively $\omega_{t}$ ) denote the restriction of this product structure to the smooth locus of the family $\mathfrak{X}$ (respectively the fiber $X_{t}$ ). In this setting we also assume:

(c) The map $\rho_{1}: X \rightarrow X_{1}$ is an isomorphism of Kähler manifolds, i.e. $\rho_{1}^{*}\left(\omega_{1}\right)=\omega$.

(d) Let $T=\left(S^{1}\right)^{n}$ denote the compact subtorus of $\mathbb{T}$. The Kähler form $\Omega$ on $\mathbb{P}^{N}$ is $T$ invariant; in particular the restriction $\omega_{0}$ to the toric variety $X_{0}$ is also a $T$-invariant Kähler form.

We can now state and prove one of the main results of this manuscript.

Theorem 5.2. Let $X$ be a smooth $n$-dimensional projective variety and let $\omega$ be a Kähler structure on $X$. Suppose that there exists a toric degeneration $\pi: \mathfrak{X} \rightarrow \mathbb{C}$ of $X$ satisfying the above conditions (a)-(d). Then:

(1) There exists a surjective continuous map $\phi: X \rightarrow X_{0}$ which is a symplectomorphism restricted to a dense open subset $U \subset X$.

(2) There exists a completely integrable system $\mu=\left(F_{1}, \ldots, F_{n}\right)$ on $(X, \omega)$, in the sense of Definition 1.1, such that its moment image $\Delta$ coincides with the moment image of $\left(X_{0}, \omega_{0}\right)$ (which is a polytope).

(3) Let $U \subset X$ be the open dense subset of $X$ from (1). Then the integrable system generates a torus action on $U$ and the inverse image $\mu^{-1}\left(\Delta^{\circ}\right)$ of the interior of $\Delta$ under the moment map $\mu: X \rightarrow \mathbb{R}^{n}$ of the integrable system lies in the open subset $U$.

Remark 5.3. For part (1) in Theorem 5.2 we do not need to assume that $X_{0}$ is a toric variety and that the Kähler form $\omega_{0}$ on it is torus invariant. 
Remark 5.4. The conditions (a)-(d) above are natural in the sense that they are satisfied in many of the known examples in the literature of constructions of toric degenerations. Indeed, the main purpose of Part 2 of this manuscript is to show that, in the general construction of toric degenerations in the context of the theory of Newton-Okounkov bodies, all the above conditions (a)-(d) are satisfied. These degenerations come from degenerating polynomials to their leading terms, often called a Gröbner or SAGBI degeneration. We also discuss concrete examples in Section 12 .

Proof of Theorem 5.2. Since the family is trivial away from 0 we know that there are no critical values of $\pi$ on the interval $(0,+\infty)$. Taking $\epsilon=1$, Theorem 4.1 tells us that the flow $\phi_{1}: U_{1} \rightarrow U_{0}$ extends to a continuous function $\phi: X_{1} \rightarrow X_{0}$. Hence we have a sequence of maps

$$
X \stackrel{\rho_{1}}{\longrightarrow} X_{1} \stackrel{\phi}{\longrightarrow} X_{0}
$$

where $\rho_{1}$ is a symplectomorphism and $\phi$ is continuous and its restriction to an open dense subset $U_{1}$ is a symplectomorphism. This proves part (1). Note that $\phi$ is surjective.

Let $\mu_{0}=\left(H_{1}, \ldots, H_{n}\right)$ be the moment map for the $T$-action on the toric variety $X_{0}$ with respect to the Kähler form $\omega_{0}$, which is a constant multiple of a Fubini-Study Kähler form on the projective space $\mathbb{P}^{N}$ (Section 1 and Remark 1.2 . Let $\Delta$ be the moment image, that is the image of $\mu_{0}$. Define a collection of functions $\left\{F_{1}, \ldots, F_{n}\right\}$ on $X$ by

$$
F_{i}=H_{i} \circ \phi \circ \rho_{1} \text {, for all } 1 \leq i \leq n
$$

We claim that the $\left\{F_{1}, \ldots, F_{n}\right\}$ form a completely integrable system on $X$ in the sense of Definition 1.1. First, by construction the functions $\left\{H_{1}, \ldots, H_{n}\right\}$ pairwise Poisson-commute with respect to $\omega_{0}$ and their differentials are linearly independent on a dense open set $U_{0}$ in $X_{0}$. Since $\phi \circ \rho_{1}$ is a symplectomorphism from $\rho_{1}^{-1}\left(U_{1}\right)$ to $U_{0}$, it follows that the $\left\{F_{1}, \ldots, F_{n}\right\}$ also pairwise Poisson-commute with respect to $\omega$ and their differentials are linearly independent on the dense open subset $\rho_{1}^{-1}\left(U_{1}\right)$ of $X$. The continuity of the $F_{i}$ follows from the continuity of $\phi \circ \rho_{1}$ on all of $X$ and the continuity of the $H_{i}$. Finally, since $X$ is compact, the image of the continuous map $\phi \circ \rho_{1}: X \rightarrow X_{0}$ is closed in $X_{0}$. On the other hand, this image contains the dense open set $U_{0}$ and hence $\phi \circ \rho_{1}$ is surjective. It follows that the image of $\left(F_{1}, \ldots, F_{n}\right): X_{1} \rightarrow \mathbb{R}^{n}$ is the same as the image of $\left(H_{1}, \ldots, H_{n}\right): X_{0} \rightarrow \mathbb{R}^{n}$, which is the polytope $\Delta=\Delta(R)$, as desired. This finishes the proof of (2). To prove (3) note that the gradient-Hamiltonian flow is undefined exactly at the singular points or the critical points of $f=\operatorname{Re}(\pi)$. By Corollary 3.3 we know that there are no critical points of $f$ on the smooth locus of $X_{0}$. The first assertion in (3) follows by noting that the set of singular points of $X_{0}$ is $T$-invariant. To prove the second assertion let $\Delta^{\circ}$ be the interior of the polytope $\Delta$. We know the the inverse image $\mu_{0}^{-1}\left(\Delta^{\circ}\right)$ is the open $\mathbb{T}$-orbit (isomorphic to $\mathbb{T}$ itself). Note that under the assumption that there are no critical points, the open $\mathbb{T}$-orbit in $X_{0}$ (hence $\mu_{0}^{-1}\left(\Delta^{\circ}\right)$ ) is contained in $U_{0}$. Now since the continuous map $\phi: X_{1} \rightarrow X_{0}$ is a homeomorphism between $U_{1}$ and $U_{0}$, it should map $\partial U_{1}$ to $\partial U_{0}$. That is, the inverse image of $U_{0}$ under $\phi$ is $U_{1}$. This proves the assertion.

Remark 5.5. As mentioned in the introduction, the same proof goes through to show that Theorem 5.2 still holds if the special fiber $X_{0}$ is a union of toric varieties and hence possibly reducible as a variety. In this case, the moment image is a union of convex polytopes. 


\section{Integrable Systems AND (GIT AND SYMPLECTIC) QUOTIENTS}

The goal of this section is to show that the construction of an integrable system using a toric degeneration in Sections 5 can be made compatible with the presence of a torus action. To accomplish this goal, however, the toric degeneration $\mathfrak{X}$ must be compatible with the torus action in the sense which will be explained below.

We use the setting and notation of Section 5. As before let $\mathbb{T}=\left(\mathbb{C}^{*}\right)^{n}$ denote the $n$ dimensional algebraic torus with lattice of characters $\mathbb{Z}^{n}$. Now suppose an algebraic subtorus $\mathbb{H} \subset \mathbb{T}$ of dimension $m$ acts on the variety $X$. We denote the maximal compact subgroup $T \cap \mathbb{H}$ of $\mathbb{H}$ by $H$.

By assumption the action of $\mathbb{T}$ on $X_{0}$ lifts to an action of $\mathbb{T}$ on $\mathbb{P}^{N} \times\{0\}$ induced by a linear action of $\mathbb{T}$ on $\mathbb{C}^{N+1}$. We let $\mathbb{H}$ act on $\mathbb{P}^{N} \times \mathbb{C}$ where it acts trivially on $\mathbb{C}$ and acts on $\mathbb{P}^{N}$ by restricting the action of $\mathbb{T}$ to $\mathbb{H}$. Hence in particular the $\mathbb{H}$-action on $X_{0}$ is the restriction of the $\mathbb{T}$-action on $X_{0}$.

We assume that the following compatibility conditions hold for the action of $\mathbb{H}$ and the family $\mathfrak{X} \subset \mathbb{P}^{N} \times \mathbb{C}$.

(i) The Kähler form $\Omega$ on $\mathbb{P}^{N}$ is invariant under the action of the real torus $H$.

(ii) The family $\mathfrak{X}$ is an $\mathbb{H}$-invariant subvariety of $\mathbb{P}^{N} \times \mathbb{C}$ and the action of $\mathbb{H}$ on $X_{1}$ coincides with the action of $\mathbb{H}$ on $X$ via the isomorphism $\rho_{1}: X_{1} \rightarrow X$. In other words, the $\mathbb{H}$-action on $\mathfrak{X}$ commutes with the $\mathbb{C}^{*}$-action on $\mathfrak{X}$.

Let $\mathfrak{X}^{s s} \subset \mathfrak{X}$ denote the set of semistable points of $\mathfrak{X}$ with respect to the $\mathbb{H}$-action, i.e. $(x, t) \in \mathfrak{X}$ for which there exists an $\mathbb{H}$-invariant polynomial $f$ on $\mathbb{C}^{N+1} \times \mathbb{C}$ and homogeneous on the $\mathbb{C}^{N+1}$ factor such that $f(x, t) \neq 0$. Recall that $\mathbb{H}$ acts on $\mathbb{C}^{N+1} \times \mathbb{C}$ by acting linearly on the first factor and acting trivially on the second factor. Let $p: \mathfrak{X}^{s s} \rightarrow \mathfrak{X}^{\prime}=\mathfrak{X}^{s s} / \mathbb{H}$ be the quotient map. Then since the action of $\mathbb{H}$ preserves fibers, one verifies that there is a map $\pi^{\prime}: \mathfrak{X}^{\prime} \rightarrow \mathbb{C}$ such that the diagram

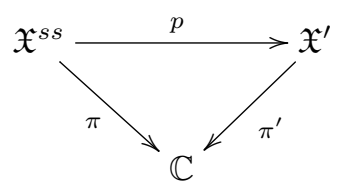

commutes. Moreover, the general fibers of $\mathfrak{X}$ and $\mathfrak{X}^{\prime}$ are $X$ and $X^{\prime}=X / / \mathbb{H}$, respectively, and the special fibers are $X_{0}$ and $X_{0}^{\prime}=X_{0} / / \mathbb{H}$, respectively.

Let $\tilde{\mu}_{H}: \mathfrak{X} \rightarrow \operatorname{Lie}(H)^{*}$ denote the moment map of the $H$-action on the family $\mathfrak{X}$ with respect to $\tilde{\omega}$. Moreover, we assume that $\tilde{\mu}_{H}$ is proper on the set of smooth points on $\mathfrak{X}, 0$ is a regular value for $\tilde{\mu}_{H}$, and $H$ acts with finite stabilizer on $\tilde{\mu}_{H}^{-1}(0)$. Then by the Kempf-Ness theorem, $\mathfrak{X}^{\prime}$ can also be identified with the symplectic quotient $\tilde{\mu}_{H}^{-1}(0) / H$.

The Kähler structure on the quotient $\mathfrak{X}^{\prime} \cong \tilde{\mu}_{H}^{-1}(0) / H$ can be described explicitly as follows. Let $(\tilde{\omega}, \tilde{g})$ denote the Kähler (symplectic) form and the corresponding Riemannian metric on $\mathfrak{X}_{\text {smooth }}$, and let $\left(\tilde{\omega}^{\prime}, \tilde{g}^{\prime}\right)$ denote the induced Kähler structure on $\mathfrak{X}_{\text {smooth }}^{\prime}$. The form $\tilde{\omega}^{\prime}$ is defined by the relation

$$
p^{*} \tilde{\omega}^{\prime}=\iota^{*} \tilde{\omega}
$$

where $p: \tilde{\mu}_{H}^{-1}(0) \rightarrow \tilde{\mu}_{H}^{-1}(0) / H \cong \mathfrak{X}^{\prime}$ is the quotient map and $\iota: \tilde{\mu}_{H}^{-1}(0) \hookrightarrow \mathfrak{X}$ denotes the inclusion. The quotient Riemannian metric $\tilde{g}^{\prime}$ is defined by the formula

$$
\tilde{g}_{x}(v, w)=\tilde{g}_{p(x)}^{\prime}\left(d p_{x}(v), d p_{x}(w)\right)
$$


where $v$ and $w$ are required to lie in the orthogonal complement to the tangent space $T_{x}(H \cdot x)$ of the $H$-orbit $H \cdot x$ through $x$.

Before proving the technical proposition leading to our Theorem 6.3, we recall a simple fact about moment maps.

Lemma 6.1. Let $K$ be a compact Lie group. Suppose $(M, \omega)$ is a Hamiltonian $K$-space with moment map $\mu_{K}: M \rightarrow \operatorname{Lie}(K)^{*}$. Let $f$ be a smooth function on $M$ which is $K$-invariant. Then the Hamiltonian flow of $f$ preserves $\mu_{K}$, i.e., $\mu_{K}$ is constant along the Hamiltonian vector field of $f$.

Let $\phi_{t}$ and $\phi_{t}^{\prime}$ denote the gradient-Hamiltonian flows on $\mathfrak{X}$ and $\mathfrak{X}^{\prime}$ respectively. Note that an immediate corollary of the above lemma is that the level set $\tilde{\mu}_{H}^{-1}(0)$ is invariant under the gradient-Hamiltonian flow $\phi_{t}$, since $\pi$ (hence $\operatorname{Re}(\pi)$ ) is $H$-invariant. Thus it makes sense to talk about the gradient-Hamiltonian flow restricted to $\tilde{\mu}_{H}^{-1}(0)$. The following proposition states that, in our setting, the gradient-Hamiltonian flow is compatible with taking GIT (symplectic) quotient.

Proposition 6.2. The quotient morphism $p: \tilde{\mu}_{H}^{-1}(0) \rightarrow \mathfrak{X}^{\prime} \cong \tilde{\mu}_{H}^{-1}(0) / H$ commutes with the gradient-Hamiltonian flows. More precisely, for any $x \in \tilde{\mu}_{H}^{-1}(0)$ and any $t>0$ such that both $\phi_{t}(x)$ and $\phi_{t}^{\prime}(p(x))$ are defined, we have

$$
p\left(\phi_{t}(x)\right)=\phi_{t}^{\prime}(p(x)) .
$$

Proof. It is enough to show that, whenever defined we have

$$
d p_{x}\left(V_{x}\right)=V_{p(x)}^{\prime}
$$

for $x \in \tilde{\mu}_{H}^{-1}(0)$. Here $V$ and $V^{\prime}$ denote the gradient-Hamiltonian vector fields of $\operatorname{Re}(\pi)$ and $\operatorname{Re}\left(\pi^{\prime}\right)$, respectively. Recall that by (2.1) we have

$$
\begin{array}{r}
V_{x}=\xi_{h}(x) /\left\|\xi_{h}(x)\right\| \\
V_{x^{\prime}}^{\prime}=\xi_{h^{\prime}}\left(x^{\prime}\right) /\left\|\xi_{h^{\prime}}\left(x^{\prime}\right)\right\|
\end{array}
$$

for $x \in \mathfrak{X}, x^{\prime} \in \mathfrak{X}^{\prime}$, where $h=\operatorname{Im}(\pi), h^{\prime}=\operatorname{Im}\left(\pi^{\prime}\right), \xi_{h}, \xi_{h^{\prime}}$ their Hamiltonian vector fields respectively, and $\|\cdot\|$ denotes the norm with respect to the metrics $\tilde{g}$ and $\tilde{g}^{\prime}$ respectively. Let $x \in \tilde{\mu}_{H}^{-1}(0) \subset \mathfrak{X}^{s s}$. By 6.2 we have $p^{*}\left(\tilde{\omega}_{p(x)}^{\prime}\right)=\tilde{\omega}_{\mid \tilde{\mu}_{H}^{-1}(0)}$. Since $h=h^{\prime} \circ p$ on $\tilde{\mu}_{H}^{-1}(0)$ by (6.1) we see that

$$
d p_{x}\left(\xi_{h}\right)=\xi_{h^{\prime}}(p(x)) .
$$

On the other hand, from the fact that $\pi$ (and hence $f=\operatorname{Re}(\pi)$ ) is $H$-invariant, we also know that the gradient $\nabla f(x)$ is orthogonal to the orbit $T_{x}(H \cdot x)$ for any $x \in \tilde{\mu}_{H}^{-1}(0)$. Recalling that $V_{x}$ is also equal to $\nabla f(x) /\|\nabla f(x)\|$, applying 6.3 to $v=w=\xi_{h}(x)$ we can conclude

$$
\left\|\xi_{h}(x)\right\|=\left\|\xi_{h^{\prime}}(p(x))\right\| .
$$

The result follows.

Let $\mu_{H}: X \rightarrow \operatorname{Lie}(H)^{*}$ denote the moment map of the $H$-action on $X$ with moment image $\Delta_{H}$ and let $\mu: X \rightarrow \mathbb{R}^{n}$ be the integrable system constructed in Theorem 5.2 with moment image $\Delta$. Then $\tilde{\mu}_{H, 1}: X_{1} \rightarrow \operatorname{Lie}(H)^{*}$ can be identified with $\mu_{H}$ via the isomorphism $\rho_{1}: X \rightarrow X_{1}$. Let $\mu_{0}$ and $\mu_{H, 0}$ denote the moment maps of $T$-action and $H$-action on the toric variety $X_{0}$ with moment images $\Delta$ and $\Delta_{H}$ respectively (Section 1). Then $\mu_{H, 0}=\tilde{\mu}_{H \mid X_{0}}$. Let $i^{*}: \operatorname{Lie}(T)^{*} \rightarrow \operatorname{Lie}(H)^{*}$ denote the dual linear map to the inclusion $i: \operatorname{Lie}(H) \hookrightarrow \operatorname{Lie}(T)$. Let $X_{0}^{\prime}:=\pi^{-1}(0)$ be the fiber over 0 of the family $\mathfrak{X}^{\prime}$. It can 
be regarded as the GIT quotient of $X_{0}$ with respect to $\mathbb{H}$. It is a possibly singular orbifold. By the Kempf-Ness theorem (for orbifolds) we can identify $X_{0}^{\prime}$ with the symplectic quotient $\mu_{H, 0}^{-1}(0) / H$. Note that $\mu_{0}$ is $T$-invariant and hence $H$-invariant and thus gives a map on $X_{0}^{\prime}=\mu_{H, 0}^{-1}(0) / H$ which we denote by $\mu_{0}^{\prime}$. Let $\Delta^{\prime}$ be the intersection of $\Delta$ with the linear subspace $\operatorname{ker}\left(i^{*}\right)$. We have a commutative diagram:

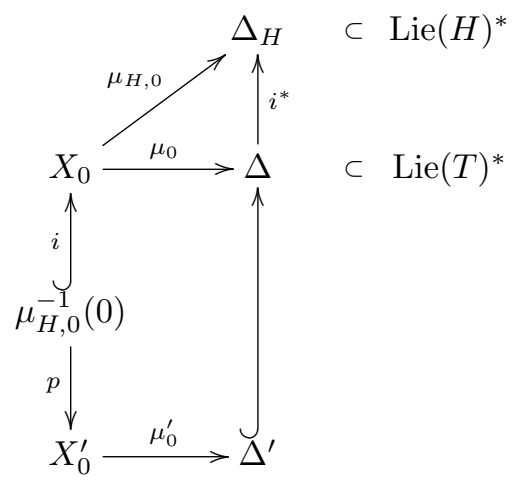

On the other hand, there are open dense subsets $U^{\prime} \subset X^{\prime}$ and $U_{0}^{\prime} \subset X_{0}^{\prime}$ and a symplectomorphism $\phi^{\prime}: U^{\prime} \rightarrow U_{0}^{\prime}$ given by the gradient-Hamiltonian flow. By Proposition 6.2 we know that $\phi^{\prime} \circ p=p \circ \phi$. Also $\phi: U \rightarrow U_{0}$ is $H$-equivariant and hence that its continuous extension $\phi: X \rightarrow X_{0}$ is also $H$-equivariant. From these one verifies that $\phi^{\prime}$ extends to a continuous map $\phi^{\prime}: X^{\prime} \rightarrow X_{0}^{\prime}$ and we have the commutative diagram:

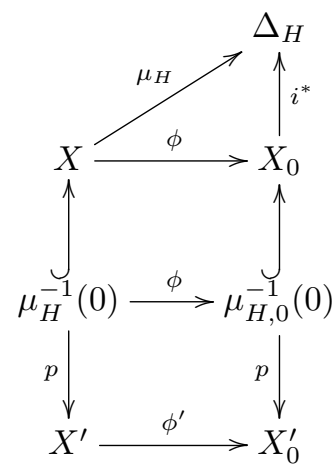

Putting 6.7 and 6.8 together we obtain the following.

Theorem 6.3. The following diagram is commutative:

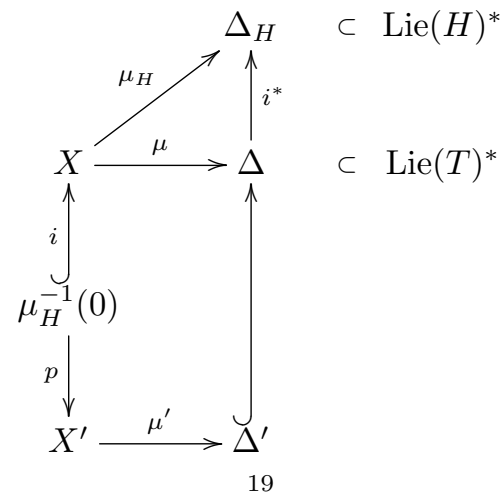




\section{Part 2. Toric degenerations from valuations and Okounkov bodies}

In Part 2 of this paper, we consider toric degenerations arising from the theory of NewtonOkounkov bodies. We will show that, for this class of toric degenerations, all of the technical assumptions we require on the toric degeneration in Part 1 (in order to construct integrable systems) are satisfied.

\section{Preliminaries for Part 2}

Let $X$ be a projective variety of dimension $n$ over $\mathbb{C}$ equipped with a very ample line bundle $\mathcal{L}$. Let $L:=H^{0}(X, \mathcal{L})$ denote the space of global sections of $\mathcal{L}$; it is a finite dimensional vector space over $\mathbb{C}$. The line bundle $\mathcal{L}$ gives rise to the Kodaira map $\Phi_{L}$ of $L$, from $X$ to the projective space $\mathbb{P}\left(L^{*}\right)$, defined as follows: the image $\Phi_{L}(x)$ of a point $x \in X$ is the point in $\mathbb{P}\left(L^{*}\right)$ corresponding to the hyperplane

$$
H_{x}=\{f \in L \mid f(x)=0\} \subset L .
$$

The assumption that $\mathcal{L}$ is very ample implies that the Kodaira map $\Phi_{L}$ is an embedding.

Alternatively we can describe $\Phi_{L}$ more concretely as follows. Let $x \in X$. Let $h \in L$ be a section of $\mathcal{L}$ with $h(x) \neq 0$; such an $h$ exists since $\mathcal{L}$ is very ample (hence $L$ has no base locus). Let $\ell_{x} \in L^{*}$ be defined by:

$$
\ell_{x}(f)=f(x) / h(x), \quad \forall f \in L .
$$

Then $\Phi_{L}(x)$ is the point in $\mathbb{P}\left(L^{*}\right)$ represented by $\ell_{x}$. It is straightforward to check that $\Phi_{L}(x)$ is independent of the choice of the section $h$.

Now let $L^{k}$ denote the image of the $k$-fold product $L \otimes \cdots \otimes L$ in $H^{0}\left(X, \mathcal{L}^{\otimes k}\right)$ under the natural map given by taking the product of sections. (In general this map may not be surjective.) The homogeneous coordinate ring of $X$ with respect to the embedding $\Phi_{L}: X \hookrightarrow \mathbb{P}\left(L^{*}\right)$ can be identified with the graded algebra

$$
R=R(L)=\bigoplus_{k \geq 0} R_{k},
$$

where $R_{k}:=L^{k}$. This is a subalgebra of the ring of sections

$$
R(\mathcal{L})=\bigoplus_{k \geq 0} H^{0}\left(X, \mathcal{L}^{\otimes k}\right) .
$$

Recall that the Hilbert function of the graded algebra $R$ is defined as $H_{R}(k):=\operatorname{dim}_{\mathbb{C}}\left(R_{k}\right)=$ $\operatorname{dim}_{\mathbb{C}}\left(L^{k}\right)$. The celebrated theorem of Hilbert on the degree of a projective variety states the following:

(1) For sufficiently large values of $k$, the function $H_{R}(k)$ coincides with a polynomial of degree $n=\operatorname{dim}_{\mathbb{C}}(X)$.

(2) Let $a_{n}:=\lim _{k \rightarrow \infty} H_{R}(k) / k^{n}$ be the leading coefficient of this polynomial. Then $n ! a_{n}$ is equal to the degree of the projective embedding of $X$ in $\mathbb{P}\left(L^{*}\right)$ (in other words, the self-intersection number of the divisor class of the line bundle $\mathcal{L}$ ).

7.1. Valuations and Newton-Okounkov bodies. Newton-Okounkov bodies give information about asymptotic behavior of Hilbert functions of graded algebras. In this section we review some background material about Newton-Okounkov bodies following KaKh08, KaKh-NO. In the present paper we will only be concerned with graded algebras which are homogeneous coordinate rings of projective varieties, as introduced in the previous section, so in the discussion below we restrict to this case. 
Following the notation of the previous section, we now wish to associate a convex body $\Delta(R) \subset \mathbb{R}^{n}$ to $R$. In the case when $X$ is a projective toric variety, there is a well-known such convex body - namely, the Newton polytope of $X$, that is, the convex hull of the weights of $T$ acting on $L$. However, for a general projective variety, we do not have such toric methods at our disposal. The tool we use for this purpose - as initially proposed by Okounkov in Ok96, Ok00] and further developed in KaKh08, KaKh-NO, LaMu09 - is a valuation on the field $\mathbb{C}(X)$ of rational functions on the variety $X$. We now recall the definition.

We fix once and for all a total order $<$ on the lattice $\mathbb{Z}^{n}$ which respects addition. Indeed, for additional concreteness, we always consider the standard lexicographic order.

Definition 7.1. A valuation on the field $\mathbb{C}(X)$ is a function $v: \mathbb{C}(X) \backslash\{0\} \rightarrow \mathbb{Z}^{n}$ satisfying the following: for any $f, g \in \mathbb{C}(X) \backslash\{0\}$,

(a) $v(f g)=v(f)+v(g)$,

(b) $v(f+g) \geq \min (v(f), v(g))$, and

(c) $v(\lambda f)=v(f)$, for all $0 \neq \lambda \in \mathbb{C}$.

Moreover, we say the valuation $v$ has one-dimensional leaves if it additionally satisfies the following:

if $v(f)=v(g)$, then there exists a non-zero constant $\lambda \neq 0 \in \mathbb{C}$ such that $v(g-\lambda f)>v(g)$ or $g-\lambda f=0$.

If $v$ is a valuation with one-dimensional leaves, then the image of $v$ is a sublattice of $\mathbb{Z}^{n}$ of full rank. Hence, by replacing $\mathbb{Z}^{n}$ with this sublattice if necessary, we will always assume without loss of generality that $v$ is surjective.

Given a variety $X$, there exist many possible valuations with one-dimensional leaves on its field of rational functions $\mathbb{C}(X)$. Below, we list several examples which arise quite naturally in geometric contexts.

Example 7.2. Let $X$ be a curve and let $p$ be a smooth point on $X$. Then

$$
v(f):=\text { order of zero or pole of } f \text { at } p
$$

defines a valuation. More generally, if $X$ is an $n$-dimensional variety, a choice of a coordinate system at a smooth point $p$ on $X$ gives a valuation on $\mathbb{C}(X)$ with one-dimensional leaves.

Example 7.3. Generalizing Example 7.2 further, we can construct a valuation out of a flag of subvarieties in $X$. More specifically, let

$$
\{p\}=X_{n} \subset \cdots \subset X_{0}=X
$$

be a sequence of closed irreducible subvarieties of $X$ such that $\operatorname{dim}_{\mathbb{C}}\left(X_{k}\right)=n-k$ for $0 \leq k \leq n$, and assume $X_{k}$ is non-singular along $X_{k+1}$ for $0 \leq k<n$ (i.e. the local ring $\mathcal{O}_{X_{k}, X_{k+1}}$ is regular). Such a sequence of subvarieties is sometimes called a Parshin point on the variety $X$. A collection $u_{1}, \ldots, u_{n}$ of rational functions on $X$ is a system of parameters about such a sequence if for each $k, u_{k \mid X_{k}}$ is a rational function on $X_{k}$ which is not identically zero and which has a zero of first order on the hypersurface $X_{k+1}$ (in other words the image of $u_{k}$ is a generator of the maximal ideal of $\mathcal{O}_{X_{k}, X_{k+1}}$ ). By the nonsingularity assumption above, such a system of parameters always exists. Given a sequence of normal subvarieties and a system of parameters $u_{1}, \ldots, u_{n}$, we can define a valuation $v$ on $\mathbb{C}(X)$ with one-dimensional leaves and values in $\mathbb{Z}^{n}$ as follows. Let $f \in \mathbb{C}(X)$ with $f \neq 0$. Then $v(f)=\left(k_{1}, \ldots, k_{n}\right)$ where the $k_{i}$ are defined inductively: the first coordinate $k_{1}$ is the order of vanishing of $f$ on $X_{1}$. Then $f_{1}=\left(u_{1}^{-k_{1}} f\right)_{\mid X_{1}}$ is a well-defined rational function on $X_{1}$ which is not identically zero, and $k_{2}$ is the order of vanishing of $f_{1}$ on $X_{2}$. Continuing in 
this manner defines all the $k_{i}$. (In fact, the normality assumption on the $X_{k}$ is not crucial; it can be avoided by passing to the normalizations.)

The following proposition is simple but fundamental; it states that the image of the valuation on a finite-dimensional subspace $E$ of $\mathbb{C}(X)$ is in one-to-one correspondence with a basis of $E$, in much the same way that the integral points in the Newton polytope of a projective toric variety $X$ correspond to a basis of $H^{0}(X, \mathcal{L})$. The proof is straightforward from the defining properties of a valuation with one-dimensional leaves.

Proposition 7.4. Let $E \subset \mathbb{C}(X)$ be a finite-dimensional subspace of $\mathbb{C}(X)$. Then $\operatorname{dim}_{\mathbb{C}}(E)=$ $\# v(E \backslash\{0\})$. In other words, the dimension of $E$ is equal to the number of values which the valuation $v$ attains on $E \backslash\{0\}$.

Fix a choice of a valuation $v$ with one-dimensional leaves on $\mathbb{C}(X)$. Using the valuation $v$ we now associate a semigroup $S(R) \subset \mathbb{N} \times \mathbb{Z}^{n}$ to the homogeneous coordinate ring $R$ of $X$. First we identify $L=H^{0}(X, \mathcal{L})$ with a (finite-dimensional) subspace of $\mathbb{C}(X)$ by choosing a non-zero element $h \in L$ and mapping $f \in L$ to the rational function $f / h \in \mathbb{C}(X)$. Similarly, we can associate the rational function $f / h^{k}$ to an element $f \in R_{k}:=L^{k} \subseteq H^{0}\left(X, \mathcal{L}^{\otimes k}\right)$. Using these identifications, we define

$$
S=S(R)=S(R, v, h)=\bigcup_{k>0}\left\{\left(k, v\left(f / h^{k}\right)\right) \mid f \in L^{k} \backslash\{0\}\right\} .
$$

From the property (a) in Definition 7.1 it follows that $S(R)$ is an additive semigroup. Moreover, from the property 7.1 in Definition 7.1 it is straightforward to show the following.

Proposition 7.5. The group generated by the semigroup $S=S(R)$, considered as a semigroup in $\mathbb{Z} \times \mathbb{Z}^{n} \cong \mathbb{Z}^{n+1}$, is (all of) $\mathbb{Z}^{n+1}$.

Remark 7.6. The semigroup $S=S(R)$ depends on the choice of valuation $v$ on $\mathbb{C}(X)$ and the section $h$. The dependence on $h$ is minor; a different choice of $h^{\prime}$ would lead to a semigroup which is shifted by the vector $k v\left(h / h^{\prime}\right)$ at the level $\{k\} \times \mathbb{Z}^{n}$. However, the dependence on the valuation $v$ is much more subtle.

In order to keep track of the natural $\mathbb{N}$-grading on the ring $R=\bigoplus_{k \geq 0} R_{k}$, it is convenient to extend the valuation $v$ to a valuation $\tilde{v}: R \backslash\{0\} \rightarrow \mathbb{N} \times \mathbb{Z}^{n}$ as follows. We define an ordering on $\mathbb{N} \times \mathbb{Z}^{n}$ by $(m, u) \leq\left(m^{\prime}, u^{\prime}\right)$ if and only if

$$
\text { either }\left(m>m^{\prime}\right) \quad \text { or } \quad\left(m=m^{\prime} \text { and } u \leq u^{\prime}\right) \text { (note the switch!) }
$$

For $f \in R$, we now define

$$
\tilde{v}(f):=\left(m, v\left(f_{m} / h^{m}\right)\right)
$$

where $f_{m}$ is the highest-degree homogeneous component of $f$. The map $\tilde{v}$ is a valuation on $R$ with the above ordering on $\mathbb{N} \times \mathbb{Z}^{n}$. By construction, the image of the valuation $\tilde{v}$ is exactly the semigroup $S=S(R)$.

Given $S \subset \mathbb{N} \times \mathbb{Z}^{n}$ an arbitrary additive semigroup, we can associate to it a convex body as follows. Let $C(S)$ denote the closure of the convex hull of $S \cup\{0\}$, considered as a subset of $\mathbb{R}_{\geq 0} \times \mathbb{R}^{n} \subset \mathbb{R}^{n+1}$. It is a closed convex cone with apex at the origin. If $S$ is a finitely generated semigroup then $C(S)$ is a rational polyhedral cone. Consider the intersection of the cone $C(S)$ with the plane $\{1\} \times \mathbb{R}^{n}$ and let $\Delta(S)$ denote the projection of this intersection to $\mathbb{R}^{n}$, via the projection on the second factor $\mathbb{R} \times \mathbb{R}^{n} \rightarrow \mathbb{R}^{n}$. Equivalently, $\Delta(S)$ can be described as

$$
\Delta(S)=\overline{\operatorname{conv}\left(\bigcup_{k>0}\{x / k \mid(k, x) \in S\}\right)} .
$$


If the cone $C(S)$ intersects the plane $\{0\} \times \mathbb{R}^{n}$ only at the origin, then the convex set $\Delta(S)$ is bounded and hence is a convex body. This will be the case for all the semigroups we consider in this paper. We refer to $\Delta(S)$ as the convex body associated to the semigroup $S$.

Definition 7.7. Let $S=S(R)$ be the semigroup associated to $(R, v, h)$ as above. We denote the cone $C(S)$ and the convex body $\Delta(S)$ by $C(R)$ and $\Delta(R)$ respectively. In this case, the cone $C(R)$ intersects $\{0\} \times \mathbb{R}^{n}$ only at the origin and hence $\Delta(R)=\Delta(R, v, h)$ is a convex body, the Okounkov body (also called Newton-Okounkov body) of $(R, v, h)$. It is also sometimes denoted as $\Delta(X, v)$ or simply $\Delta(X)$ when we wish to emphasize the underlying projective variety $X$.

Remark 7.8. From Remark 7.6 it follows that a different choice $h^{\prime} \in L$ would yield a Newton-Okounkov body $\Delta\left(R, v, h^{\prime}\right)$ which is shifted by the fixed vector $v\left(h / h^{\prime}\right)$. Thus, similar to the case of $S(R)$, the dependence of the Newton-Okounkov body on the choice of section $h$ is minor. However, as in Remark 7.6 the dependence of $\Delta(R, v)$ on the valuation $v$ is more subtle.

The Newton-Okounkov body $\Delta(R)$ encodes information about the asymptotic behavior of the Hilbert function of $R$ (see [KaKh08, KaKh-NO, [LaMu09] and Ok96, Ok00]). Let $H_{R}(k):=\operatorname{dim}_{\mathbb{C}}\left(R_{k}\right)$ be the Hilbert function of the graded algebra $R$.

Theorem 7.9. The Newton-Okounkov body $\Delta(R)$ has real dimension $n$, and the leading coefficient

$$
a_{n}=\lim _{k \rightarrow \infty} \frac{H_{R}(k)}{k^{n}},
$$

of the Hilbert function of $R$ is equal to $\operatorname{Vol}_{n}(\Delta(R))$, the Euclidean volume of $\Delta(R)$ in $\mathbb{R}^{n}$. In particular, the degree of the projective embedding of $X$ in $\mathbb{P}\left(L^{*}\right)$ is equal to $n ! \operatorname{Vol}_{n}(\Delta(R))$.

Remark 7.10. In KaKh-NO, the asymptotic behavior of Hilbert functions of a much more general class of graded algebras is addressed, namely, graded algebras of the form $A=\bigoplus_{k \geq 0} A_{k}$ such that

- $A_{k} \subset \mathbb{C}(X)$ for all $k$, and

- $A$ is contained in a finitely generated algebra of the same form.

Note that such an algebra $A$ is not necessarily finitely generated. This class of graded algebras includes arbitrary graded linear series on a variety, and in particular, the ring of sections of arbitrary line bundles (not just ample ones).

\section{A tORIC DEGENERATION ASSOCiATED to A VALUATION}

As in Section 7, let $X$ be an $n$-dimensional projective variety with a very ample line bundle $\mathcal{L}$, the corresponding homogeneous coordinate ring $R$, a valuation $v$ with one-dimensional leaves and the value semigroup $S=S(R, v, h)$. Moreover, from now on, we place the additional assumption that:

$$
S \text { is finitely generated. }
$$

This assumption implies that the Newton-Okounkov body $\Delta(R)$ is a rational polytope. The semigroup algebra $\mathbb{C}[S]$ of a semigroup $S \subseteq \mathbb{N} \times \mathbb{Z}^{n}$ is defined to be the subalgebra of $\mathbb{C}\left[t, x_{1}, \ldots, x_{n}\right]$ spanned by the monomials $t^{k} x_{1}^{a_{1}} \cdots x_{n}^{a_{n}}$ for all $\left(k, a_{1}, \ldots, a_{n}\right) \in S$. Recall that $S$ is the image of the extended valuation $\tilde{v}: R \backslash\{0\} \rightarrow \mathbb{N} \times \mathbb{Z}^{n}$ of (7.4). In KaKh08, the authors mentioned briefly that the homogeneous coordinate ring $R$ of $X$ can be degenerated in a flat family to the semigroup algebra $\mathbb{C}[S]$ associated to the value semigroup $S=S(R)$. In fact, Teissier proved already in 1999 that there always exists a toric degeneration from 
an algebra to the semigroup algebra associated to a valuation Te99. (More generally, the existence of a degeneration of an algebra to its associated graded with respect to a filtration is well-known and classical in commutative algebra [Ei95, Chapter 6].) In the context of Newton-Okounkov bodies, this idea was developed further, with suggestions on applications to other areas such as Schubert calculus, in An13. Geometrically, the existence of the algebraic degeneration means that the variety $X$ can be degenerated, in a flat family $\mathfrak{X}$, to the (not necessarily normal) projective toric variety $X_{0}$ associated to the semigroup $S$. The normalization of $X_{0}$ is then the (normal) toric variety corresponding to the polytope $\Delta(R)$.

Our construction of an integrable system uses Anderson's toric degeneration as a key ingredient, so in this section we both review and slightly expand on his exposition in An13. First we recall how the semigroup algebra $\mathbb{C}[S]$ of $R$ is related to the data of the extended valuation $\tilde{v}$. The link comes from a filtration of $R$ defined using $\tilde{v}$ as follows. Let

$$
R_{\geq(m, u)}:=\{f \in R \mid \tilde{v}(f) \geq(m, u) \text { or } f=0\}
$$

and similarly for $R_{>(m, u)}$. By the definition of the valuation $v$ and the definition of the order (7.3) on $\mathbb{N} \times \mathbb{Z}^{n}$, each $R_{\geq(m, u)}$ (and $R_{>(m, u)}$ ) is a (finite-dimensional) vector subspace of $R$. Moreover, again by properties of valuations, for any $(m, u)$ and $\left(m^{\prime}, u^{\prime}\right)$, we have

$$
R_{\geq(m, u)} \cdot R_{\geq\left(m^{\prime}, u^{\prime}\right)} \subseteq R_{\geq\left(m+m^{\prime}, u+u^{\prime}\right)} .
$$

We can now define the associated graded $\operatorname{ring} \operatorname{gr} R$ with respect to this filtration as

$$
\operatorname{gr} R:=\bigoplus_{(m, u)} R_{\geq(m, u)} / R_{>(m, u)} .
$$

This is naturally an $S$-graded ring. In fact, since $\tilde{v}$ has one-dimensional leaves, the spaces $R_{\geq(m, u)} / R_{>(m, u)}$ have dimension 1 precisely when $(m, u) \in S$, and 0 otherwise. Since the homogeneous elements of $\operatorname{gr} R$ are not zero divisors, one shows that $\operatorname{gr} R$ is isomorphic to the semigroup algebra $\mathbb{C}[S]$ (see [BG09, Remark 4.13]).

As mentioned above, there is a well-known method, namely the Rees algebra construction, of degenerating an algebra $R$ to its associated graded $\operatorname{gr} R$ corresponding to a filtration of $R$ in a flat family. This is explained in more detail in [An13. Alternatively, one can describe the same degeneration using a choice of a collection of sections $\left\{f_{i j}\right\} \in R$ as we now explain. This choice gives rise to an embedding of the degenerating family in a weighted projective space, which we use in our geometric constructions.

Definition 8.1. Let $R=\bigoplus_{i} R_{i}$ and $\tilde{v}$ as above. We say that a set $\mathcal{B} \subset R$ is a Khovanskii basis ${ }^{2}$ for $(R, \tilde{v})$ if the set of images $\tilde{v}(\mathcal{B})=\{\tilde{v}(f) \mid f \in \mathcal{B}\}$ generate the semigroup $S=S(R)=S(R, v, h)$.

This definition generalizes the notion of a SAGBI (Subalgebra Analogue of Gröbner Basis for Ideals) basis for a subalgebra of a polynomial ring, and was introduced in Ka11 and Ma11. In general a Khovanskii basis may or may not be finite.

Since we assumed that $S$ is finitely generated $R$ has a finite Khovanskii basis $\mathcal{B}$. Without loss of generality and by adding more elements to $R$ if necessary, we can assume that $\mathcal{B}$ consists of homogeneous elements $f_{i j}, 1 \leq i \leq r, 1 \leq j \leq n_{i}$, with the following additional properties:

(a) $f_{i j} \in R_{i}$ for all $1 \leq i \leq r, 1 \leq j \leq n_{i}$, and

\footnotetext{
${ }^{2}$ The name "Khovanskii basis" was suggested by B. Sturmfels in honor of A. G. Khovanskii's influential contributions to combinatorial algebraic geometry.
} 
(b) for each $i$, the collection $\left\{f_{i 1}, f_{i 2}, \ldots, f_{i n_{i}}\right\}$ is a vector space (i.e. additive) basis for $R_{i}$.

For the remainder of this discussion, we fix a finite Khovanskii basis $\left\{f_{i j}\right\}_{1 \leq i \leq r, 1 \leq j \leq n_{i}}$ of $R$ satisfying the conditions (a) and (b) above. Since the $\tilde{v}\left(f_{i j}\right)$ generate the semigroup $S(R)$, by a standard argument using the classical subduction algorithm, we can show that every element of $R$ can be represented as a polynomial in the $f_{i j}$. For the sake of completeness we include a proof.

Proposition 8.2. Any $f \in R$ can be written as a polynomial in the $f_{i j}$. In other words, a finite Khovanskii basis $\left\{f_{i j}\right\}$ of $R$ generates $R$ as an algebra.

Proof. First we observe that in the value semigroup $S=S(R, \tilde{v})$ every increasing sequence of elements eventually stabilizes. Let $\left(m_{1}, u_{1}\right) \leq\left(m_{2}, u_{2}\right) \leq \cdots$ be an increasing sequence in $S$. Then from the definition of $\leq$ on $\mathbb{N} \times \mathbb{Z}^{n}$ (see 77.3 ) we have $m_{1} \geq m_{2} \geq \cdots$. Since $\mathbb{N}$ is well-ordered and each $S_{i}$ is finite we see that for $i$ sufficiently large $\left(m_{i}, u_{i}\right)=$ $\left(m_{i+1}, u_{i+1}\right)=\cdots$ as claimed. Now take $0 \neq h \in R$. Write $\tilde{v}(h)=\sum_{i=1}^{t} k_{i} \tilde{v}\left(f_{i}\right)$. Since $v$ (and hence $\tilde{v}$ ) have one-dimensional leaves, there exists a nonzero constant $\lambda \in \mathbb{C}$ such that $g_{1}:=h-c f_{1}^{k_{1}} \cdots f_{t}^{k_{t}}$ has the property that either $g_{1}=0$ or $\tilde{v}\left(g_{1}\right)>\tilde{v}(h)$. If $g_{1}=0$ we are done. Otherwise, repeating the same argument for $g_{1}$ in place of $h$, we obtain a sequence $\tilde{v}(h)<\tilde{v}\left(g_{1}\right)<\tilde{v}\left(g_{2}\right)<\cdots$. Since an increasing sequence in $S$ eventually stabilizes, we see that at some point we must have $g_{i}=0$. This finishes the proof.

We can now state the main result of this section [An13, Proposition 5.1].

Theorem 8.3. Let $R$ be as above and assume $S=S(R)$ is finitely generated. Then there is a finitely generated, $\mathbb{N}$-graded, flat $\mathbb{C}[t]$-subalgebra $\mathcal{R} \subset R[t]$, such that:

(a) $\mathcal{R}\left[t^{-1}\right] \cong R\left[t, t^{-1}\right]$ as $\mathbb{C}\left[t, t^{-1}\right]$-algebras, and

(b) $\mathcal{R} / t \mathcal{R} \cong \operatorname{gr} R$.

The geometric interpretation An13, Corollary 5.3] of Theorem 8.3 is obtained by taking Proj with respect to the $\mathbb{N}$-grading on $\mathcal{R}$.

Corollary 8.4. There is a projective flat family $\pi: \mathfrak{X}:=\operatorname{Proj} \mathcal{R} \rightarrow \mathbb{C}$ such that:

(a) For any $z \neq 0$ the fiber $X_{z}=\pi^{-1}(z)$ is isomorphic to $X=$ Proj $R$. More precisely, the family over $\mathbb{C} \backslash\{0\}$, i.e. $\pi^{-1}(\mathbb{C} \backslash\{0\})$ is isomorphic to $X \times(\mathbb{C} \backslash\{0\})$.

(b) The special fiber $X_{0}=\pi^{-1}(0)$ is isomorphic to $\operatorname{Proj}(\operatorname{gr} R) \cong \operatorname{Proj} \mathbb{C}[S]$ and is equipped with an action of $\left(\mathbb{C}^{*}\right)^{n}$, where $n=\operatorname{dim}_{\mathbb{C}} X$. The normalization of the variety $\operatorname{Proj}(\operatorname{gr} R)$ is the toric variety associated to the rational polytope $\Delta(R)$.

Sketch of proof of Theorem 8.3. Let $\tilde{v}\left(f_{i j}\right)=\left(i, u_{i j}\right)$. Let $\bar{f}_{i j}$ denote the image of $f_{i j}$ in the associated graded gr $R$. Let $A=\mathbb{C}\left[x_{i j}\right]$ denote the polynomial algebra in the indeterminates $x_{i j}, 1 \leq i \leq r, 1 \leq j \leq n_{i}$. Define an $\left(\mathbb{N} \times \mathbb{Z}^{n}\right)$-grading on $A$ by $\operatorname{deg}\left(x_{i j}\right):=\left(i, u_{i j}\right)$; thus the surjective map $A \rightarrow \operatorname{gr} R$ defined by $x_{i j} \mapsto \bar{f}_{i j}$ is a map of graded rings. The kernel of this map is a homogeneous ideal $I_{0}$. Let $\bar{g}_{1}, \ldots, \bar{g}_{q}$ be homogeneous generators for the kernel and let $\operatorname{deg}\left(\bar{g}_{k}\right)=\left(n_{k}, v_{k}\right)$. It follows that $\bar{g}_{k}\left(f_{11}, \ldots, f_{r n_{r}}\right)$ lies in $R_{>\left(n_{k}, v_{k}\right)}$ for each k. By the proof of Proposition 8.2 one can find elements $g_{k} \in \bar{g}_{k}+A_{<\left(n_{k}, v_{k}\right)}$ such that $g_{k}\left(f_{11}, \ldots, f_{r n_{r}}\right)=0$. The $g_{k}$ will not be homogeneous for the full $\mathbb{N} \times \mathbb{Z}^{n}$ grading of $A$, but since the $f_{i j}$ are homogeneous for the first $\mathbb{N}$ factor, the $g_{k}$ can be chosen to respect the $\mathbb{N}$-grading as well.

The induced map $A /\left\langle g_{1}, \ldots, g_{q}\right\rangle \rightarrow R$ is an isomorphism. In fact, if $I$ denotes the kernel of the map $A \rightarrow R$, then $g_{k} \in I$ by construction, and the initial terms $g_{k}$ generate the 
initial ideal $I_{0}$ of $I$ (which is the kernel of $A \rightarrow \operatorname{gr} R$ ). It follows that the $g_{k}$ 's form a Gröbner basis for $I$, with respect to the term order determined by the order on $\mathbb{Z} \times \mathbb{Z}^{n}$, cf. [Ei95, Exercise 15.14(a)]. (Specifically, the term order is $\prod_{i j} x_{i j}^{a_{i j}} \leq \prod_{i j} x_{i j}^{b_{i j}}$ if and only if $\sum_{i j} a_{i j}\left(i, u_{i j}\right) \leq \sum_{i j} b_{i j}\left(i, u_{i j}\right)$. Note that we allow ties between monomials in this notion of term order.)

The remainder of the construction and proof will only be sketched, see An13 for details. Here we record only the points directly relevant to our construction in Section 10. Let $\mathcal{S} \subset \mathbb{Z} \times \mathbb{Z}^{n}$ be a finite subset consisting of all the degrees $(s, v)$ for all the monomials appearing in any of the $g_{k}$. Let $p: \mathbb{Z} \times \mathbb{Z}^{n} \rightarrow \mathbb{Z}$ be a linear map which preserves the ordering on $\mathcal{S}$ (cf. [An13, Lemma 5.2, Proof of Prop. 5.1]). Let $w_{i j}=p\left(i, u_{i j}\right)$ be the degree of $x_{i j}$ under the weighting induced by $p$. From the choice of $p$ it follows that for any $k$, the initial term of $g_{k}$ with respect to the weighting defined by $p$ is exactly $\bar{g}_{k}$. Let $\ell_{k}=p\left(n_{k}, v_{k}\right)$, and set

$$
\tilde{g}_{k}=\tau^{\ell_{k}} g_{k}\left(\tau^{-w_{11}} x_{11}, \ldots, \tau^{-w_{r n_{r}}} x_{r n_{r}}\right) \in A[\tau],
$$

where $\tau$ is an indeterminate. Consider the homomorphism $A[\tau] \rightarrow R[t]$ given by

$$
x_{i j} \mapsto t^{w_{i j}} f_{i j} \quad \text { and } \tau \mapsto t .
$$

Let $\mathcal{R}$ be the image of this homomorphism; this ring satisfies all the conditions of the theorem. In addition, the ring $\mathcal{R}$ can be presented explicitly as $\mathcal{R}=A[\tau] /\left(\tilde{g}_{1}, \ldots, \tilde{g}_{q}\right)$. (This is a standard way of producing a Rees ring from a Gröbner basis; see, e.g., Ei95, Theorem 15.17].)

The results just recounted yield a projective flat family $\mathfrak{X}$ associated to $R$ and $S(R, v, h)$. In fact, we will see below, in Section 9, that the choice of a Khovanskii basis $\left\{f_{i j}\right\}$ above naturally gives rise to an explicit embedding of the family into $W \mathbb{P} \times \mathbb{C}$, where $W \mathbb{P}$ denotes a weighted projective space, and the restriction of the standard projection $W \mathbb{P} \times \mathbb{C} \rightarrow \mathbb{C}$ to the family $\mathfrak{X}$ is exactly the projection $\pi: \mathfrak{X} \rightarrow \mathbb{C}$ of Corollary 8.4. By embedding the weighted projective space $W \mathbb{P}$ into a "large" projective space $\mathbb{P}\left(\mathbf{V}_{d}^{*}\right)$ we then obtain an embedding of the family $\mathfrak{X}$ into $\mathbb{P}\left(\mathbf{V}_{d}^{*}\right) \times \mathbb{C}$. Our construction of an integrable system on the original variety $X$ will depend on this embedding in the sense that we use a Kähler structure on $\mathfrak{X}$ which is the restriction of a Kähler structure on $\mathbb{P}\left(\mathbf{V}_{d}^{*}\right) \times \mathbb{C}$, judiciously chosen to be compatible with the original Kähler structure on $X$ as well as the (compact) torus-invariant Kähler structure on the toric variety $X_{0}$. The details are the subject of the next section.

As a final remark, we note that if $X$ is assumed to be smooth, then by Corollary 8.4(a) we know that the family $\mathfrak{X}$ is nonsingular away from the special fibre $X_{0}$. In fact, by Proposition 3.1 we know that more is true. We have the following.

Corollary 8.5. Let $X, R, \tilde{v}, S$ and $\mathfrak{X}$ be as above. Suppose that $X$ is smooth. Then the family $\mathfrak{X}$ is nonsingular away from the singular locus of the special fiber $X_{0}$.

\section{A KäHLer structure on the FAMily $\mathfrak{X}$}

As usual let $X$ be an $n$-dimensional projective variety. From now on in addition we assume that

$$
X \text { is smooth. }
$$

Let $\mathcal{L}$ be a very ample line bundle on $X$ with the corresponding embedding $X \hookrightarrow \mathbb{P}\left(L^{*}\right)$ where $L=H^{0}(X, \mathcal{L})$. We fix a Kähler structure $\omega$ on $X$ by restrcting a Fubini-Study Kähler structure $\Omega_{H}$ on the projective space $\mathbb{P}\left(L^{*}\right)$ with respect to a Hermitian product $H$ on $L^{*}$ (see e.g. GrHa78). 
We follow the notation and assumptions of Section 8 in particular that the value semigroup $S$ is finitely generated. As mentioned at the end of Section 8 , an explicit choice of a finite Khovanskii basis $\left\{f_{i j}\right\}$ naturally gives rise to an embedding of the toric degeneration $\mathfrak{X}$ constructed in Corollary 8.4 into $\mathbb{P}\left(\mathbf{V}_{d}^{*}\right) \times \mathbb{C}$ for an appropriate projective space $\mathbb{P}\left(\mathbf{V}_{d}^{*}\right)$ (to be defined concretely below). This embedding allows us to build an extension of the Kähler structure on the original variety $X$ (the generic fiber of $\mathfrak{X}$ ) to all of $\mathfrak{X}$, in such a way that the extension restricts to a torus-invariant Kähler structure on the special fiber $X_{0}$. We now explain the details.

9.1. Weighted projective spaces. Let $R$ and $S(R, v, h)$ be as in Section 8 In general, a Khovanskii basis $\left\{f_{i j}\right\}$ has elements of $R$ of different homogeneous degrees. Thus the choice of $\left\{f_{i j}\right\}$ naturally leads to an embedding of the family $\mathfrak{X}$ into a weighted projective space $W \mathbb{P}$ (we recall the definition below). In the simple case when the Khovanskii basis consists only of elements of degree 1 , the weighted projective space is an ordinary projective space. In addition, in the next section we embed the weighted projective space into a large (ordinary) projective space $\mathbb{P}\left(\mathbf{V}_{d}^{*}\right)$. Thus it is not strictly logically necessary to discuss the weighted projective spaces (since we may simply embed $X$ directly into $\mathbb{P}\left(\mathbf{V}_{d}^{*}\right)$ ) but we find it more natural to first discuss the map $X \rightarrow W \mathbb{P}$ which takes account of the degrees of the $f_{i j}$.

We first recall the definition and some basic facts about weighted projective spaces. Let $V_{1}, \ldots, V_{r}$ be finite dimensional vector spaces over $\mathbb{C}$ and $m_{1}, \ldots, m_{r}$ positive integers. Consider the action of $\mathbb{C}^{*}$ on $V_{1} \times \cdots \times V_{r}$ by:

$$
\lambda \cdot\left(v_{1}, \ldots, v_{r}\right)=\left(\lambda^{m_{1}} v_{1}, \ldots, \lambda^{m_{r}} v_{r}\right) .
$$

We define the weighted projective space $W \mathbb{P}=W \mathbb{P}\left(V_{1}, \ldots, V_{r} ; m_{1}, \ldots, m_{r}\right)$ to be the quotient of $\left(V_{1} \times \cdots \times V_{r}\right) \backslash\{(0, \ldots, 0)\}$ by this $\mathbb{C}^{*}$-action. The point in $W \mathbb{P}$ represented by $\left(v_{1}, \ldots, v_{r}\right) \in V_{1} \times \cdots \times V_{r}$ is denoted $\left(v_{1}: \cdots: v_{r}\right)$. There is a natural rational map $W \mathbb{P} \rightarrow \mathbb{P}\left(V_{1}\right) \times \cdots \times \mathbb{P}\left(V_{r}\right)$ given by:

$$
\left(v_{1}: \cdots: v_{r}\right) \mapsto\left(\left[v_{1}\right], \ldots,\left[v_{r}\right]\right) .
$$

This is defined whenever all the $v_{i}$ are nonzero. The following is well-known.

Theorem 9.1. The weighted projective space $W \mathbb{P}$ is a normal projective variety. Moreover, $W \mathbb{P}$ is smooth if and only if $m_{1}=\cdots=m_{r}$.

9.2. Embedding a projective variety into a weighted projective space. Returning now to the general setup of Section 7, let $X$ be a projective variety equipped with a very ample line bundle $\mathcal{L}$ and let $L=H^{0}(X, \mathcal{L})$. Let $r>0$ be a positive integer. For any $k$ with $1 \leq k \leq r$, define $V_{k}:=\left(L^{k}\right)^{*}$ and $m_{k}:=k$. Consider the associated weighted projective space

$$
W \mathbb{P}=W \mathbb{P}\left(L^{*},\left(L^{2}\right)^{*}, \ldots,\left(L^{r}\right)^{*} ; 1,2, \ldots, r\right) .
$$

Analogous to the construction of the Kodaira map $\Phi_{L}$, we can also define a morphism $\Psi: X \rightarrow W \mathbb{P}$ as follows. Let $x \in X$. Let $h \in L$ be a section with $h(x) \neq 0$; as before, since $L$ has no base locus, such an $h$ always exists. For each $k$ with $1 \leq k \leq r$, let $\ell_{x, k}$ be the element in $\left(L^{k}\right)^{*}$ defined by

$$
\ell_{x, k}(f):=f(x) / h^{k}(x), \quad \forall f \in L^{k} .
$$

Define $\Psi(x)$ to be the point $\left(\ell_{x, 1}: \cdots: \ell_{x, r}\right) \in W \mathbb{P}$. It can be checked that $\Psi(x)$ is independent of the choice of the section $h$ and is a morphism from $X$ to $W \mathbb{P}$. Note that each $\Phi_{L^{k}}$ is an embedding, so $\Psi$ is also an embedding. 
The morphism $\Psi$ can also be described explicitly in terms of coordinates (at least on an open dense subset) as follows. Fix bases $\left\{f_{i 1}, \ldots, f_{i n_{i}}\right\}$ for the $L^{i}$. With respect to the corresponding dual bases for the $\left.\left(L^{i}\right)^{*}\right)$, the map $\Psi$ can be given in coordinates by

$$
\Psi: x \mapsto\left(\left(\frac{f_{11}(x)}{h(x)}, \ldots, \frac{f_{1 n_{1}}(x)}{h(x)}\right): \cdots:\left(\frac{f_{r 1}(x)}{h^{r}(x)}, \ldots, \frac{f_{r n_{r}}(x)}{h^{r}(x)}\right)\right)
$$

at the points $x \in X$ where $h(x) \neq 0$.

With this notation in place, we take a moment to concretely and geometrically interpret the family $\mathfrak{X}$ constructed in Section 8 as a subvariety of $W \mathbb{P} \times \mathbb{C}$; this embedding is determined by the map (8.1). Specifically, consider the map

$$
\rho: X \times \mathbb{C}^{*} \rightarrow \mathfrak{X} \hookrightarrow W \mathbb{P} \times \mathbb{C}=W \mathbb{P}\left(L^{*},\left(L^{2}\right)^{*}, \ldots,\left(L^{r}\right)^{*} ; 1,2, \ldots, r\right) \times \mathbb{C}
$$

given by

$$
\left.(x, t) \mapsto\left(\left(t^{w_{11}} \frac{f_{11}(x)}{h(x)}, \ldots, t^{w_{1 n_{1}}} \frac{f_{1 n_{1}}(x)}{h(x)}\right): \cdots:\left(t^{w_{r 1}} \frac{f_{1 r}(x)}{h^{r}(x)}, \ldots, t^{w_{r n_{r}}} \frac{f_{r n_{r}}(x)}{h^{r}(x)}\right)\right), t\right)
$$

where $h$ is a section with $h(x) \neq 0$ (see 9.1$)$. The image of the family $\mathfrak{X}$ sitting inside the weighted projective space $W \mathbb{P} \times \mathbb{C}$ is then the closure of the image of this map. This image is cut out by the equations $\tilde{g}_{k}\left(x_{i j}, \tau\right)=0$ for $k=1, \ldots, q$. The projection $\pi: \mathfrak{X} \rightarrow \mathbb{C}$ of Corollary 8.4 is then the projection $\mathfrak{X} \subseteq W \mathbb{P} \times \mathbb{C} \rightarrow \mathbb{C}$ to the second factor.

Remark 9.2. By construction, the image of $X_{0}=\pi^{-1}(0)$ in the weighted projective space is cut out by the equations $\bar{g}_{k}\left(x_{i j}\right)=0$ for $1 \leq k \leq q$. The map $\mathbb{C}\left[x_{i j}\right] \rightarrow \mathbb{C}\left[x_{i j}\right] / I_{0} \cong \operatorname{gr} R$ is a homomorphism of $\left(\mathbb{N} \times \mathbb{Z}^{n}\right)$-graded algebras and hence the embedding $X_{0} \hookrightarrow W \mathbb{P} \times\{0\}$ is $\mathbb{T}=\left(\mathbb{C}^{*}\right)^{n}$ equivariant.

9.3. Embedding a weighted projective space in a large projective space. In order to construct a Kähler structure on the family $\mathfrak{X}$, it will be useful to embed the (usually singular) weighted projective space $W \mathbb{P}$ into a "large" classical (and hence smooth) projective space. In this section we describe a particular such embedding.

Let $V_{1}, \ldots, V_{r}$ be finite-dimensional vector spaces over $\mathbb{C}$ and $m_{1}, \ldots, m_{r}$ positive integers as in Section 9.1. Let $\operatorname{Sym}\left(\left(V_{1} \times \cdots \times V_{r}\right)^{*}\right)$ denote the algebra of polynomials on $V_{1} \times \cdots \times V_{r}$. We equip $\operatorname{Sym}\left(\left(V_{1} \times \cdots \times V_{r}\right)^{*}\right) \cong \operatorname{Sym}\left(V_{1}^{*} \times V_{2}^{*} \times \cdots \times V_{r}^{*}\right)$ with a grading by assigning degree $m_{i}$ to any $f_{i}^{*} \in V_{i}^{*}$. Let $d>0$ be a positive integer. With respect to the above grading, let $\mathbf{V}_{d}$ denote the subspace of $\operatorname{Sym}\left(\left(V_{1} \times \cdots \times V_{r}\right)^{*}\right)$ consisting of the elements of total degree $d$. From the definition of the grading it then follows that

$$
\mathbf{V}_{d} \cong \bigoplus_{\substack{\beta=\left(\beta_{1}, \ldots, \beta_{r}\right) \in \mathbb{Z}_{\geq 0}^{r} \\ \sum_{i} m_{i} \beta_{i}=d}} \bigotimes_{i=1}^{r} \operatorname{Sym}_{\beta_{i}}\left(V_{i}^{*}\right)
$$

where $\operatorname{Sym}_{\beta_{i}}\left(V_{i}^{*}\right)$ denotes the space of homogeneous polynomials of degree $\beta_{i}$ on $V_{i}$.

Given $v=\left(v_{1}, \ldots, v_{r}\right) \in V_{1} \times \cdots \times V_{r}$, the evaluation map

$$
\ell_{v}: p \mapsto p(v)
$$

defines a linear function $\ell_{v}$ on $\operatorname{Sym}\left(\left(V_{1} \times \cdots \times V_{r}\right)^{*}\right)$. It follows that the map $v \mapsto \ell_{v \mid \mathbf{V}_{d}}$ induces a rational map

$$
\Theta: W \mathbb{P}\left(V_{1}, \ldots, V_{r}\right) \rightarrow \mathbb{P}\left(\mathbf{V}_{d}^{*}\right) .
$$

Moreover, we have the following Do82.

Proposition 9.3. If $d=\prod_{i=1}^{r} m_{i}$ then $\Theta$ is a morphism and an embedding. 
The map $\Theta$ can also be described concretely in coordinates. Suppose $n_{i}=\operatorname{dim}_{\mathbb{C}}\left(V_{i}\right)$ for each $i$ and fix a basis for each $V_{i}$; let $\left(x_{i 1}, \ldots, x_{i n_{i}}\right)$ denote the corresponding coordinates for $V_{i}$ (i.e. dual vectors in $\left.V_{i}^{*}\right)$. Then, under the identification $\operatorname{Sym}\left(\left(V_{1} \times \cdots \times V_{r}\right)^{*}\right) \cong$ $\operatorname{Sym}\left(V_{1}^{*} \times \cdots \times V_{r}^{*}\right) \cong \mathbb{C}\left[x_{i j} ; 1 \leq i \leq r, 1 \leq j \leq n_{i}\right]$, the set of monomials

$$
\left\{x_{11}^{\alpha_{11}} \cdots x_{r n_{r}}^{\alpha_{r n_{r}}} \mid \sum_{i=1}^{r} m_{i} \sum_{j=1}^{n_{i}} \alpha_{i j}=d, \alpha_{i j} \in \mathbb{Z}_{\geq 0}\right\}
$$

form a basis for $\mathbf{V}_{d}$. With respect to these coordinates, the morphism $\Theta: W \mathbb{P} \rightarrow \mathbb{P}\left(\mathbf{V}_{d}^{*}\right)$ can be described explicitly as

$$
\Theta:\left(\left(x_{11}, \ldots, x_{1 n_{1}}\right): \cdots:\left(x_{r 1}, \ldots, x_{r n_{r}}\right)\right) \mapsto\left(x_{11}^{\alpha_{11}} \cdots x_{r n_{r}}^{\alpha_{r n_{r}}} \mid \sum_{i=1}^{r} \sum_{j=1}^{n_{i}} m_{i} \alpha_{i j}=d, \alpha_{i j} \in \mathbb{Z}_{\geq 0}\right) .
$$

The following will be used in Section 11 .

Remark 9.4. Let $\mathbb{H} \cong\left(\mathbb{C}^{*}\right)^{m}$ be an algebraic torus. Suppose $\mathbb{H}$ acts linearly on each $V_{i}$. The diagonal $\mathbb{H}$-action on $V_{1} \times \cdots \times V_{r}$ induces an action of $\mathbb{H}$ on $W \mathbb{P}\left(V_{1}, \ldots, V_{r} ; m_{1}, \ldots, m_{r}\right)$ and on $\mathbb{P}\left(\mathbf{V}_{d}^{*}\right)$. It is not hard to see that $\Theta$ is $\mathbb{H}$-equivariant with respect to these actions.

Consider now the special case in Section 9.2 so $V_{k}=\left(L_{k}\right)^{*}$ and $m_{k}=k$. In this setting, $\mathbf{V}_{d}$ is the subspace of $\operatorname{Sym}\left(L \times \cdots \times L^{r}\right) \cong \operatorname{Sym}\left(\left(L^{*} \times \cdots \times\left(L^{r}\right)^{*}\right)^{*}\right)$ consisting of homogeneous elements of degree $d$. Let $d=r$ !. Proposition 9.3 then implies that the composition $\Theta \circ \Psi: X \rightarrow \mathbb{P}\left(\mathbf{V}_{d}^{*}\right)$ is an embedding. Again we take a moment to describe $\Theta \circ \Psi$ explicitly in terms of coordinates. As before, let $x \in X$ and let $h \in L$ such that $h(x) \neq 0$. Using the same bases $\left\{f_{i 1}, \ldots, f_{i n_{i}}\right\}$ for $L^{i}$ as above, note that any $p \in \mathbf{V}_{d}$ can be written as

$$
p=\sum_{\substack{\alpha=\left(\alpha_{11}, \ldots, \alpha_{1 n_{1}}, \ldots, \alpha_{r 1}, \ldots, \alpha_{r n_{r}}\right), \alpha_{i j} \in \mathbb{Z}_{\geq 0} \\ \sum_{i=1}^{r} i\left(\sum_{j=1}^{n_{i}} \alpha_{i j}\right)=d=r !}} c_{\alpha} f_{11}^{\alpha_{11}} \cdots f_{1 n_{1}}^{\alpha_{1 n_{1}}} \cdots f_{r 1}^{\alpha_{r 1}} \cdots f_{r n_{r}}^{\alpha_{r n_{r}}} .
$$

From the previous coordinate descriptions of $\Theta$ and $\Psi$ it is now straightforward to see that $(\Theta \circ \Psi)(x) \in \mathbb{P}\left(\mathbf{V}_{d}^{*}\right)$ can be described as the point

$$
\left(\frac{f_{11}(x)^{\alpha_{11}} \cdots f_{r n_{r}}(x)^{\alpha_{r n_{r}}}}{h(x)^{d}} \mid \sum_{i=1}^{r} i\left(\sum_{j=1}^{n_{i}} \alpha_{i j}\right)=d=r !, \alpha_{i j} \in \mathbb{Z}_{\geq 0}\right)
$$

on the locus where $h(x) \neq 0$.

For each $i>0$, the sum and product of sections give a natural linear map from $L^{\otimes i}$ onto $L^{i}$. Similarly there is a natural linear map from $\mathbf{V}_{d}$ onto $L^{d}$. These induce embeddings $\left(L^{i}\right)^{*} \hookrightarrow\left(L^{\otimes i}\right)^{*}$ and $\left(L^{d}\right)^{*} \hookrightarrow \mathbf{V}_{d}^{*}$, which in turn give embeddings of the corresponding projective spaces $\mathbb{P}\left(\left(L^{i}\right)^{*}\right) \hookrightarrow \mathbb{P}\left(\left(L^{\otimes i}\right)^{*}\right)$ and $j: \mathbb{P}\left(\left(L^{d}\right)^{*}\right) \hookrightarrow \mathbb{P}\left(\mathbf{V}_{d}^{*}\right)$. From 9.6) and the definition of the Kodaira map $\Phi_{L^{d}}$, it follows that the diagram

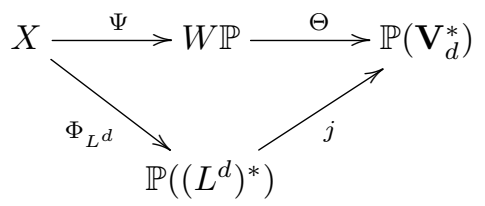

commutes. 
The following will be used in Section 11 .

Remark 9.5. Suppose $\mathbb{H}$ is an algebraic torus. Suppose $\mathbb{H}$ acts on $X$ and that the very ample line bundle $\mathcal{L}$ is $\mathbb{H}$-linearized. Then, for any $k>0$, the spaces $\left(L^{k}\right)^{*}$ are $\mathbb{H}$-modules and hence $\mathbb{H}$ also acts on $W \mathbb{P}$ and $\mathbb{P}\left(\mathbf{V}_{d}^{*}\right)$ as in Remark 9.4. It can be checked that all the maps in the diagram (9.7) are $\mathbb{H}$-equivariant for the corresponding $\mathbb{H}$-actions.

9.4. A Kähler structure on $\mathbb{P}\left(\mathbf{V}_{d}^{*}\right)$. In order to define a Kähler structure $\tilde{\omega}$ on the family $\mathfrak{X}$, we first choose an appropriate Kähler structure on $\mathbb{P}\left(\mathbf{V}_{d}^{*}\right)$. Recall that the Kähler structure $\omega$ on $X$ is defined as the pull-back of a Fubini-Study form $\Omega_{H}$ on $\mathbb{P}\left(L^{*}\right)$ with respect to a fixed Hermitian product $H$ on $L^{*}$. In this section we wish to construct a Kähler structure on the projective space $\mathbb{P}\left(\mathbf{V}_{d}^{*}\right)$ such that its pull-back under the embedding $\Theta \circ \Psi$ coincides with the form $\omega$ on $X$.

For this purpose, it is convenient to extend the diagram 9.7). We maintain the notation of Sections 9.3 and 9.2 . Suppose $\beta=\left(\beta_{1}, \ldots, \beta_{r}\right) \in \mathbb{Z}_{\geq 0}^{r}$ where $\sum_{i} i \cdot \beta_{i}=d$, as in Section 9.3. Identifying $L^{\otimes d} \cong \otimes_{i=1}^{r} L^{\otimes i \cdot \beta_{i}}$ in the natural way, consider the map

$$
L^{\otimes d} \rightarrow \bigotimes_{i=1}^{r} \operatorname{Sym}_{\beta_{i}}\left(L^{i}\right)
$$

induced by taking the natural map $L^{\otimes i \cdot \beta_{i}} \rightarrow \operatorname{Sym}_{\beta_{i}}\left(L^{i}\right)$, induced by the product of sections, on each factor. Taking the direct sum of 9.8 over all such $\beta$ we obtain a natural map

$$
\bigoplus_{\substack{\beta=\left(\beta_{1}, \ldots, \beta_{r}\right) \in \mathbb{Z}_{\geq 0}^{r} \\ \sum_{i=1}^{r} i \cdot \beta_{i}=d}} L^{\otimes d} \rightarrow \mathbf{V}_{d}
$$

using the identification $(9.3)$. Since $\sqrt{9.9}$ is induced from taking the product of sections, it is straightforward to see that 9.9 fits into a commutative diagram

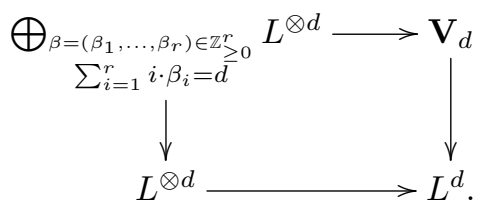

where the left vertical map takes the sum of the components in the direct sum, the top horizontal arrow is $(9.9)$, and the other two arrows are the natural ones induced from the sum and product of sections. It is clear that all four maps are surjective.

Taking duals, we get the diagram of inclusions

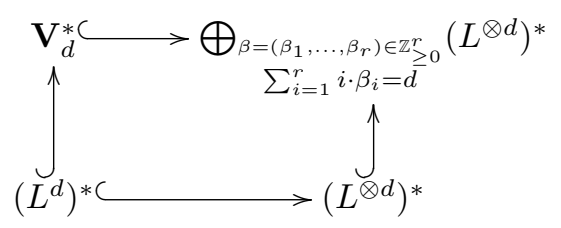

where the right vertical map is given by the diagonal inclusion $v \mapsto(v, v, \ldots, v)$. Considering the induced morphisms between the corresponding projective spaces and putting this 
together with (9.7), we obtain the commutative diagram

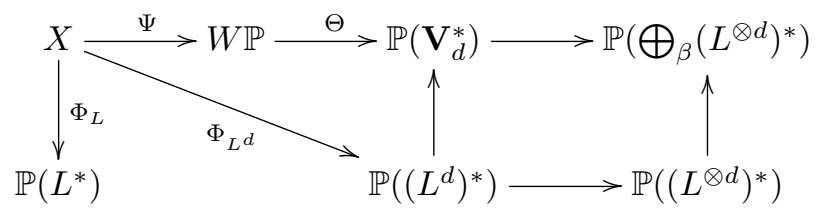

where all the maps are embeddings.

We will use the Hermitian product $H$ on $L^{*}$ to define a Hermitian product $\mathbf{H}$ on the vector space $\bigoplus_{\beta}\left(L^{\otimes d}\right)^{*}$. First we recall the following. Let $H_{1}, H_{2}$ be Hermitian products on vector spaces $V_{1}, V_{2}$ respectively. Then $H_{1} \oplus H_{2}$ and $H_{1} \otimes H_{2}$, defined respectively by the formulas

$$
\begin{aligned}
& \left(H_{1} \oplus H_{2}\right)\left(\left(v_{1}, v_{2}\right),\left(w_{1}, w_{2}\right)\right)=H_{1}\left(v_{1}, w_{1}\right)+H_{2}\left(v_{2}, w_{2}\right) \\
& \left(H_{1} \otimes H_{2}\right)\left(v_{1} \otimes v_{2}, w_{1} \otimes w_{2}\right)=H_{1}\left(v_{1}, w_{1}\right) H_{2}\left(v_{2}, w_{2}\right),
\end{aligned}
$$

for all $v_{i}, w_{i} \in V_{i}$, yield well-defined Hermitian products on $V_{1} \oplus V_{2}$ and $V_{1} \otimes V_{2}$ respectively. Recalling also that $\left(L^{\otimes d}\right)^{*} \cong\left(L^{*}\right)^{\otimes d}$, we now define

$$
\mathbf{H}:=\bigoplus_{\substack{\beta=\left(\beta_{1}, \ldots, \beta_{r}\right) \in \mathbb{Z}_{\geq 0}^{r} \\ \sum_{i=1}^{r} i \cdot \beta_{i}=\bar{d}}} H^{\otimes d}
$$

on $\bigoplus_{\beta} L^{\otimes d}$. By restriction $\mathbf{H}$ induces Hermitian products on the other three spaces in the diagram 9.10). The Hermitian product on $\left(L^{\otimes d}\right)^{*} \cong\left(L^{*}\right)^{\otimes d}$ induced from the diagonal inclusion $v \mapsto(v, v, \ldots, v)$ coincides with the Hermitian product $N \cdot H^{\otimes d}$ where here $N$ denotes the number of components in the direct sum $\bigoplus_{\beta} L^{\otimes d}$. We now define

$$
\boldsymbol{\Omega}:=\frac{1}{N \cdot d} \Omega_{\mathbf{H}}
$$

where $\Omega_{\mathbf{H}}$ is the Kähler form on $\mathbb{P}\left(\bigoplus_{\beta}\left(L^{\otimes d}\right)^{*}\right)$ corresponding to the Hermitian product $\mathbf{H}$.

The normalization factor in (9.14) is chosen precisely so that the following holds.

Theorem 9.6. The pull-back of the form $\Omega$ on $\mathbb{P}\left(\bigoplus_{\beta}\left(L^{\otimes d}\right)^{*}\right)$ to $X$, via the maps in (9.11), coincides with the original Kähler form $\omega$ with respect to the Hermitian product $H$ on $L^{*}$.

Proof. By what was said above, the pull-back of $\Omega$ to $\mathbb{P}\left(\left(L^{d}\right)^{*}\right)$ is equal to $(1 / N \cdot d) \Omega_{H \otimes d}$, where $\Omega_{H \otimes d}$ denotes the Kähler form on $\mathbb{P}\left(\left(L^{d}\right)^{*}\right)$ induced from $H^{\otimes d}$. The theorem now follows from the fact that the pull-back of $\Omega_{H^{\otimes d}}$ to $X$ under the Kodaira map $\Phi_{L^{d}}$ is equal to $d \cdot \omega$ (see KaKh10, Lemma 7.9]). Recall $\omega$ is the pullback of $\Omega_{H}$ to $X$ under $\Phi_{L}$. Since $\Omega_{H}$ pulls back to $\mathbb{P}\left(\left(L^{d}\right)^{*}\right)$ to equal $N \cdot \Omega_{H^{\otimes d}}$, the claim follows from 9.14.

Finally, we address the invariance of the Kähler form $\boldsymbol{\Omega}$ under a torus action. Let $\mathbb{T} \cong\left(\mathbb{C}^{*}\right)^{n}$ and let $T=\left(S^{1}\right)^{n}$ denote the usual compact torus in $\mathbb{T}$. Suppose $\mathbb{T}$ acts linearly on each space $\left(L^{i}\right)^{*}$ such that the Hermitian product $H^{\otimes i}$ on $\left(L^{i}\right)^{*}$ is $T$-invariant. A natural geometric situation when this arises is when $\mathbb{T}$ acts on $X$ and $\mathcal{L}$ is a $\mathbb{T}$-linearized line bundle equipped with a $T$-invariant Hermitian form. Note that the $\mathbb{T}$-actions on the $\left(L^{i}\right)^{*}$ induce a $\mathbb{T}$-action on $\mathbf{V}_{d}^{*}$.

Proposition 9.7. Suppose $T$ acts linearly on the $L^{i}$ and suppose for each $i$ the $T$-action on $L^{i}$ preserves $H^{\otimes i}$. Then the Hermitian product $\mathbf{H}$ on $\mathbf{V}_{d}^{*}$ is $T$-invariant. In particular, the corresponding Kähler form $\boldsymbol{\Omega}$ on $\mathbb{P}\left(\mathbf{V}_{d}^{*}\right)$ is T-invariant. 
Proof. Let $T$ act on a vector space $V$ equipped with a Hermitian product $H$. Then the $T$-action preserves $H$ if and only if there exists an orthonormal basis of $V$ consisting of $T$-weight vectors. For each $i$, let $\left\{f_{i 1}^{*}, \ldots, f_{i n_{i}}^{*}\right\}$ be a basis consisting of $T$-weight vectors. By assumption, the $T$-action preserves $H^{\otimes i}$, so we may assume without loss of generality that the basis is orthonormal. Under the identification $\operatorname{Sym}\left(\left(L^{1}\right)^{*} \otimes\left(L^{2}\right)^{*} \otimes \cdots \otimes\left(L^{r}\right)^{*}\right) \cong$ $\mathbb{C}\left[f_{i j} ; 1 \leq i \leq r, 1 \leq j \leq n_{i}\right]$ (compare (9.4) and (9.5p), the set of vectors

$$
\left.\mathbf{B}=\left\{\prod_{i, j}\left(f_{i j}^{*}\right)^{\alpha_{i j}} \mid \alpha=\left(\alpha_{i j}\right) ; \sum_{i=1}^{r} i \cdot \sum_{j=1}^{n_{i}} \alpha_{i j}=d ; \alpha_{i j} \in \mathbb{Z}_{\geq 0}\right)\right\}
$$

is a basis of $T$-weight vectors for $\mathbf{V}_{d}^{*}$. Moreover, from the definition of $\mathbf{H}$, it follows that $\mathbf{B}$ is an orthonormal basis, as desired.

9.5. Kähler structure on the family $\mathfrak{X}$. We are finally prepared to construct the Kähler structure on the toric degeneration $\mathfrak{X}$ constructed in Section 8 . Recall that $X$ is a smooth projective variety equipped with $\mathcal{L}$ a very ample line bundle and we set $L=H^{0}(X, \mathcal{L})$ and let $R$ denote its homogeneous coordinate ring. Let $v$ be a valuation with one-dimensional leaves on $\mathbb{C}(X)$ and assume that the associated semigroup $S=S(R)$ is finitely generated. Also let $\Delta=\Delta(R)$ denote the associated Newton-Okounkov body. Since $S$ is finitely generated, $\Delta$ is a rational polytope.

In Section 8 we described a flat family $\mathfrak{X}$ such that general fibers $X_{z}, z \neq 0$, are isomorphic to $X$ and the central fiber $X_{0}$ is the (possibly non-normal) toric variety associated to the semigroup $S$. Recall from Section 9.2 (in particular the map $\rho$ described in $(9.2)$ ) that the family $\mathfrak{X}$ lies in $W \mathbb{P} \times \mathbb{C}$ where the weighted projective space $W \mathbb{P}$ is $W \mathbb{P}\left(L^{*}, \ldots,\left(L^{r}\right)^{*} ; 1, \ldots, r\right)$ for some $r>0$. By embedding $W \mathbb{P}$ in a usual projective space $\mathbb{P}\left(\mathbf{V}_{d}^{*}\right)$ as in Section 9.3 , we may therefore think of the family $\mathfrak{X}$ as a subvariety in the smooth variety $\mathbb{P}\left(\mathbf{V}_{d}^{*}\right) \times \mathbb{C}$. Let $\Omega$ denote the Kähler structure on $\mathbb{P}\left(\mathbf{V}_{d}^{*}\right)$ constructed in Section 9.4 As in Part 1 we equip $\mathbb{P}\left(\mathbf{V}_{d}^{*}\right) \times \mathbb{C}$ with the product symplectic structure $\Omega \times\left(\frac{i}{2} d z \wedge d \bar{z}\right)$ where $z$ is the complex parameter on the $\mathbb{C}$ factor and so $\frac{i}{2} d z \wedge d \bar{z}$ is the standard Kähler form on $\mathbb{C}$. We denote by $\tilde{\omega}$ (respectively $\omega_{z}$ ) the restriction of this product structure to the smooth locus of the family $\mathfrak{X}$ (respectively the fiber $X_{z}$ ). For $z \neq 0,\left(X_{z}, \omega_{z}\right)$ is a smooth Kähler manifold. When we write $\omega_{0}$, we mean the Kähler form on the smooth locus of $X_{0}$.

Recall that the construction of the family $\mathfrak{X}$ involved a choice of elements $\left\{f_{i j}\right\}$ in $R$, satisfying properties (a)-(c) in Definition 8.1. The following lemma proves that we may also assume, without loss of generality, that in addition to the properties (a)-(c), the collection $\left\{f_{i j}\right\}$ is compatible with the choice of a Hermitian metric.

Lemma 9.8. Let $v: \mathbb{C}(X) \backslash\{0\} \rightarrow \mathbb{Z}^{n}$ be a valuation with one-dimensional leaves. Let $V \subset \mathbb{C}(X)$ be a finite-dimensional vector subspace and let $H$ be a Hermitian product on the dual space $V^{*}$. Then there exists a basis $\left\{b_{1}, \ldots, b_{k}\right\}$ of $V$ such that

(1) the corresponding dual basis $\left\{b_{1}^{*}, \ldots, b_{k}^{*}\right\}$ is orthonormal with respect to $H$ and

(2) $v\left(b_{i}\right) \neq v\left(b_{j}\right)$ for all $i \neq j$, i.e., $v(V \backslash\{0\})=\left\{v\left(b_{1}\right), \ldots, v\left(b_{k}\right)\right\}$.

Proof. From Proposition 7.4 we know that $\# v(V \backslash\{0\})=\operatorname{dim}_{\mathbb{C}} V$. Let $k=\operatorname{dim}_{\mathbb{C}} V$ and let $\left\{a_{1}>\cdots>a_{k}\right\}=v(V \backslash\{0\})$, listed in decreasing order. For $a \in \mathbb{Z}^{n}$ let $V_{\geq a}:=\{x \mid v(x) \geq$ $a\} \cup\{0\}$. This is a subspace of $V$. We have a flag of subspaces

$$
\{0\} \varsubsetneqq V_{\geq a_{1}} \varsubsetneqq \cdots \varsubsetneqq V_{\geq a_{k}}=V .
$$

Using the Hermitian product on $V^{*}$, we define an identification $\phi: V^{*} \rightarrow V$ which thus also equips $V$ with a Hermitian product by pullback via $\phi$. In such a situation an orthonormal 
basis of $V$ is identified via $\phi$ with its own dual basis in $V^{*}$, which is also orthonormal. Thus, the desired basis can be constructed by finding an orthonormal basis of $V_{\geq a_{1}}$, then inductively an orthonormal basis for $V_{\geq a_{s}}$ extending the given one on $V_{\geq a_{s-1}}$.

From this lemma it follows that we may assume that our Khovanskii basis $\left\{f_{i j}\right\}$ has the property that, for each fixed $i, 1 \leq i \leq r$, the collection $\left\{f_{i j}\right\}_{1 \leq j \leq n_{i}}$ is a basis for $L^{i}$ such that its dual basis $\left\{f_{i 1}^{*}, \ldots, f_{i n_{i}}^{*}\right\}$ is orthonormal in $\left(L^{i}\right)^{*}$, with respect to the Hermitian product on $\left(L^{i}\right)^{*}$ induced from $H^{\otimes i}$.

Now let the torus $\mathbb{T}$ act linearly on each $\left(L^{i}\right)^{*}$ such that every $f_{i j}^{*}$ is a $T$-weight vector with weight $u_{i j}=v\left(f_{i j}\right)$. Note this corresponds to the action on $W \mathbb{P}\left(L^{*},\left(L^{2}\right)^{*}, \ldots,\left(L^{r}\right)^{*} ; 1,2, \ldots, r\right)$ (see Remark 9.4). From the orthogonality of the basis $\left\{f_{i 1}^{*}, \ldots, f_{i n_{i}}^{*}\right\}$ it follows that the induced action of $T$ on $\left(L^{i}\right)^{*}$ preserves the Hermitian product.

Recall that for $0 \neq z \in \mathbb{C}, \rho_{z}$ denotes the restriction of the embedding $\rho: X \times \mathbb{C}^{*} \rightarrow \mathfrak{X} \subset$ $W \mathbb{P} \times \mathbb{C}$ given in 9.2 to $X \times\{z\}$.

Proposition 9.9. We have:

(a) Under the isomorphism $\rho_{1}: X \rightarrow X_{1} \subset \mathfrak{X}$, the pullback $\rho_{1}^{*} \omega_{1}$ of the Kähler form $\omega_{1}$ on $X_{1}$ is equal to the original Kähler form $\omega$ on $X$.

(b) The Kähler form $\omega_{0}$ on the (smooth locus of) the $\mathbb{T}$-toric variety $X_{0} \subset \mathbb{P}\left(\mathbf{V}_{d}^{*}\right) \times\{0\} \cong$ $\mathbb{P}\left(\mathbf{V}_{d}^{*}\right)$ is invariant under the T-action.

Proof. The embedding

$$
\rho_{1}: X \hookrightarrow W \mathbb{P} \times\{1\}
$$

coincides with the embedding $\Psi: X \rightarrow W \mathbb{P}$ in diagram (9.7). This proves (a). Part (b) follows from Proposition 9.7 where $\mathbb{T}$ acts on each space $L^{i}$ in such a way that the $f_{i j}$ are weight vectors with weights $u_{i j}$.

\section{InTEGRABLE SYSTEMS CONSTRUCTED FROM A VALUATION}

We may now state the main theorem of Part 2 of this manuscript. The results from the previous sections make the proof quite straightforward. We use the setting of Section 9.4 . namely that $X$ is an $n$-dimensional complex projective variety equipped with $\mathcal{L}$ a very ample Hermitian line bundle on $X$, with corresponding Kähler form $\omega$ obtained via the Kodaira embedding. We let $R$ denote the ring of sections of the line bundle $\mathcal{L}$. We take a valuation $v: \mathbb{C}(X) \backslash\{0\} \rightarrow \mathbb{Z}^{n}$ with one-dimensional leaves, where $\mathbb{Z}^{n}$ is equipped with a total order. Then $S=S(R)$ and $\Delta=\Delta(R)$ denote the value semigroup and the Newton-Okounkov body of $(R, v)$ respectively. In this setting we have the following.

Theorem 10.1. With notation as above, suppose that $(X, \omega)$ is smooth and the semigroup $S=S(R)$ is finitely generated.

(1) There exists a completely integrable system $\mu:\left(F_{1}, \ldots, F_{n}\right) \rightarrow \mathbb{R}^{n}$ on $X$ in the sense of Definition 1.1, i.e.:

(a) The functions $F_{1}, \ldots, F_{n}$ are continuous on all of $X$.

(b) There exists an open dense subset $U$ such that $F_{1}, \ldots, F_{n}$ are differentiable on $U$, and the differentials $d F_{1}, \ldots, d F_{n}$ are linearly independent on $U$.

(c) $F_{1}, \ldots, F_{n}$ pairwise Poisson-commute on $U \subset X$.

Moreover, the image of $\mu$ coincides with the Newton-Okounkov body $\Delta=\Delta(R)$.

(2) The integrable system in (1) generates a torus action on the dense open subset $U$ of $X$. Moreover, the inverse image of the interior of $\Delta$ lies in the open subset $U$. 
Proof. Corollary 8.4 . Corollary 8.5 and Proposition 9.9 show that the conditions (a)-(d) in Theorem 5.2 are satisfied for the toric degeneration constructed from the valuation $v$, equipped with the Kähler structure $\tilde{\omega}$ built in Section 9.5. Thus Theorem 10.1 follows from Theorem 5.2 .

\section{GIT AND SYMPLECTIC QUOTIENTS}

In this section we discuss the compatibility of the flat family constructed in Section 8 (out of the data of a valuation with a finitely generated value semigroup) with a torus action. We will show that the family can be constructed so that the compatibility conditions in Section 6 are satisfied and hence the integrable system from Section 10 descends to the quotient by the torus. The material in this section is straightforward, but we spell out the details for completeness.

11.1. Quotients of toric varieties. $\mathbb{T}=\left(\mathbb{C}^{*}\right)^{n}$ denotes, as before, the $n$-dimensional algebraic torus with lattice of characters $\mathbb{Z}^{n}$. Consider a finitely generated semigroup $S \subset$ $\mathbb{N} \times \mathbb{Z}^{n}$ of integral points. We will assume that $S$ generates the whole lattice $\mathbb{Z}^{n+1}=\mathbb{Z} \times \mathbb{Z}^{n}$ and also $S_{1}=S \cap\left(\{1\} \times \mathbb{Z}^{n}\right) \neq \emptyset$. The semigroup $S$ gives rise to the semigroup algebra $\mathbb{C}[S]$. The algebra $\mathbb{C}[S]$ is the subagebra of the algebra of polynomials $\mathbb{C}\left[t, x_{1}, \ldots, x_{n}\right]$ consisting of polynomials whose monomials belong to the semigroup $S$. That is,

$$
\mathbb{C}[S]=\left\{f \in \mathbb{C}\left[t, x_{1}, \ldots, x_{n}\right] \mid f=\sum_{(k, \alpha) \in S} c_{(k, \alpha)} t^{k} x^{\alpha}\right\} .
$$

Here as usual $x^{\alpha}$ is shorthand for $x_{1}^{a_{1}} \cdots x_{n}^{a_{n}}$. The projection of $S$ on the first factor gives a grading on the algebra $\mathbb{C}[S]$ (by non-negative integers) and the corresponding variety $\operatorname{Proj}(\mathbb{C}[S])$ is a projective $\mathbb{T}$-toric variety.

Now suppose $\mathbb{H} \subset \mathbb{T}$ is an algebraic subtorus of dimension $m$ with lattice of characters $M$. The inclusion $\mathbb{H} \subset \mathbb{T}$ gives a surjective homomorphism $\lambda: \mathbb{Z}^{n} \rightarrow M$, which in turn extends to a linear map $\lambda_{\mathbb{R}}: \mathbb{R}^{n} \rightarrow M_{\mathbb{R}}=M \otimes \mathbb{R}$. Let $\tilde{\lambda}: \mathbb{Z}^{n+1} \rightarrow M$ be a homomorphism such that the restriction of $\tilde{\lambda}$ to $\{0\} \times \mathbb{Z}^{n}$ coincides with $\lambda$. Fix $\left(1, a_{0}\right) \in S$ and define $\lambda_{0}:=\tilde{\lambda}\left(1, a_{0}\right)$. Then for any $(k, a) \in \mathbb{Z}^{n+1}$, the fact that $\tilde{\lambda}$ is a homomorphism implies

$$
\tilde{\lambda}(k, a)=\lambda\left(a-k a_{0}\right)+k \lambda_{0}
$$

so the lift $\tilde{\lambda}$ is determined by $\lambda$ and by its value at $\left(1, a_{0}\right)$. We will return to this point later. The lifted homomorphism $\tilde{\lambda}$ gives an $M$-grading on the semigroup algebra $\mathbb{C}[S]$ where for $(k, a) \in S$, the monomial $t^{k} x^{a}$ has degree $\tilde{\lambda}(k, a)$. We can uniquely extend $\tilde{\lambda}$ to a linear function $\tilde{\lambda}_{\mathbb{R}}: \mathbb{R}^{n+1} \rightarrow M_{\mathbb{R}}$. Let $\Lambda_{\mathbb{R}}^{\prime} \subset \mathbb{R}^{n+1}$ denote the kernel of $\tilde{\lambda}_{\mathbb{R}}$ and let $S^{\prime}=S \cap \Lambda_{\mathbb{R}}^{\prime}$ be the subsemigroup of $S$ obtained by intersecting with the subspace $\Lambda_{\mathbb{R}}^{\prime}$. The kernel of the linear map $\lambda_{\mathbb{R}}$ has dimension $n-m$ and hence the subspace $\Lambda_{\mathbb{R}}^{\prime}$ has dimension $n-m+1$.

Lemma 11.1. Let $S$ and $S^{\prime}$ be as above, and let $\Delta=\Delta(S), \Delta^{\prime}=\Delta\left(S^{\prime}\right)$ be the convex bodies associated to the semigroups $S$ and $S^{\prime}$ respectively (see (7.5)). Then:

(a) $S^{\prime}$ is a finitely generated semigroup.

(b) $\left(\{1\} \times \Delta^{\prime}\right)=(\{1\} \times \Delta) \cap \Lambda_{\mathbb{R}}^{\prime}$.

Proof. Part (b) of the Lemma follows immediately from the definitions. Part (a) is a consequence of Gordan's Lemma (see e.g. CoLiSc11, Proposition 2.17]).

The $M$-grading on $\mathbb{C}[S]$ gives rise to an $\mathbb{H}$-action on the $\mathbb{T}$-toric variety $X_{S}:=\operatorname{Proj} \mathbb{C}[S]$. By construction, the $\mathbb{H}$-invariant subalgebra $\mathbb{C}[S]^{\mathbb{H}}$, which is the homogeneous-degree-0 part of $\mathbb{C}[S]$ with respect to the $M$-grading, is the semigroup algebra $\mathbb{C}\left[S^{\prime}\right]$. Let $X_{S}^{\prime}:=\operatorname{Proj} \mathbb{C}\left[S^{\prime}\right]$. 
The algebra $\mathbb{C}\left[S^{\prime}\right]$ is $\mathbb{N} \times \mathbb{Z}^{n}$ graded and hence the variety $X_{S}^{\prime}$ has a $\mathbb{T}$-action. By definition the variety $X_{S}^{\prime}$ is the GIT quotient of the variety $X_{S}$ with respect to the $\mathbb{H}$-action. In fact, it is a toric variety for the action of a torus $\mathbb{T}^{\prime}$ which is a quotient of $\mathbb{T}$ (by a subgroup containing, but possibly larger than, $\mathbb{H}$ ). The complex dimension of the variety $X_{S}^{\prime}$ (and the torus $\left.\mathbb{T}^{\prime}\right)$ is equal to the real dimension of the polytope $\Delta^{\prime}$.

Fix a set of generators $\left(i, u_{i j}\right)$ for $S$. Embed $X_{S}$ in a weighted projective space $W \mathbb{P}$. Let $W \mathbb{P} \hookrightarrow \mathbb{P}\left(V_{d}^{*}\right)$ be embedding of this weighted projective space in a large projective space. We equip $X_{S} \hookrightarrow \mathbb{P}\left(V_{d}^{*}\right)$ with a symplectic structure as in Section 1. Let $\mu_{H}: X_{S} \rightarrow \operatorname{Lie}(H)^{*}=$ $M_{\mathbb{R}}, \mu_{T}: X_{S} \rightarrow \operatorname{Lie}(T)^{*} \cong \mathbb{R}^{n}$ denote the moment maps for the actions of the compact tori $H \subset \mathbb{H}$ and $T \subset \mathbb{T}$, respectively, on $X_{S}$. Recall that by the Kempf-Ness theorem the GIT quotient $X_{S}^{\prime}$ can also be realized as the symplectic quotient $\mu_{H}^{-1}(0) / H$ of $X_{S}$ at 0 , provided that 0 is a regular value of $\mu_{H}$ and $H$ acts freely on $\mu_{H}^{-1}(0)$. The GIT quotient $X_{S}^{\prime}$ inherits a symplectic (in fact Kähler) structure coinciding with the quotient symplectic structure on $X_{S}^{\prime}$ [MuFoKi94, Section 8.3]. Let $\mu_{T^{\prime}}: X_{S}^{\prime} \rightarrow \operatorname{Lie}\left(T^{\prime}\right)^{*}$ denote the moment map of $X_{S}^{\prime}$ regarded as a Hamiltonian $T^{\prime}$-space. Then the following diagram

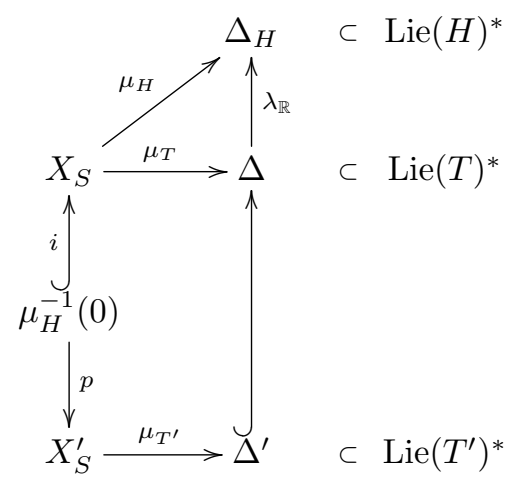

commutes, where $i: \mu_{H}^{-1}(0) \hookrightarrow X_{S}$ is the inclusion map, and $p: \mu_{H}^{-1}(0) \rightarrow \mu_{H}^{-1}(0) / H=X_{S}^{\prime}$ is the symplectic quotient map. Moreover, the image of $\operatorname{Lie}\left(T^{\prime}\right)^{*}$ in $\operatorname{Lie}(T)^{*}$ lies in the kernel of the linear map $\lambda_{\mathbb{R}}$.

11.2. Invariant valuations. We now consider a more general situation. As usual let $\mathbb{H} \cong$ $\left(\mathbb{C}^{*}\right)^{m}$ denote an $m$-dimensional algebraic torus with character group $M \cong \mathbb{Z}^{m}$. Let $X$ be a projective $\mathbb{H}$-variety of dimension $n$. Note in particular that we do not assume $X$ is an $\mathbb{H}$-toric variety, and it may be that $m$ is strictly less than $n$. The $\mathbb{H}$-action on $X$ induces an $\mathbb{H}$-action on the field of rational functions $\mathbb{C}(X)$.

Suppose $\mathcal{L}$ is an $\mathbb{H}$-linearized very ample line bundle on $X$. Then $L=H^{0}(X, \mathcal{L})$ is a finite-dimensional $\mathbb{H}$-module and the homogeneous coordinate ring $R=R(L)$ is a graded $\mathbb{H}$-algebra. We define the weight semigroup of $R$ to be

$$
S_{\mathbb{H}}(R):=\left\{(k, \lambda) \mid \text { there exists } f \in R_{k} \text { with } t \cdot f=t^{\lambda} f \text { for all } t \in \mathbb{H}\right\} \subset \mathbb{N} \times M \text {. }
$$

If $R$ is finitely generated as an algebra, then $S_{\mathbb{H}}(R)$ is a finitely generated semigroup. Hence the convex body $\Delta_{\mathbb{H}}:=\Delta\left(S_{\mathbb{H}}(R)\right)$ associated via 7.5 to the semigroup $S_{\mathbb{H}}(R)$ is a rational polytope. Following [Br86] and $\left[\mathrm{KaKh}-\mathrm{CB}\right.$, we call $\Delta_{\mathbb{H}}$ the moment polytope of the $\mathbb{H}$-algebra $R$. The terminology is motivated from the well-known fact that the polytope $\Delta_{\mathbb{H}}$ coincides with the (closure of the) image of the moment map of $X_{\text {smooth }}$ regarded as a Hamiltonian $H$-space with respect to an $H$-invariant Kähler structure on $\mathbb{P}\left(L^{*}\right)$ (here $H \subset \mathbb{H}$ denotes the maximal compact torus in $\mathbb{H})$. 
We now fix an $\mathbb{H}$-invariant valuation $v: \mathbb{C}(X) \backslash\{0\} \rightarrow \mathbb{Z}^{n}$ with one-dimensional leaves. Such a valuation always exists (see $[\mathrm{Ok} 96, \mathrm{KaKh}-\mathrm{CB}]$ ). Let $h \neq 0$ in $L$ be a $\mathbb{T}$-weight vector of weight $\lambda_{0}$. Following the method in Section 7.1, we now define $\tilde{v}: R \backslash\{0\} \rightarrow \mathbb{N} \times \mathbb{Z}^{n}$ by

$$
\tilde{v}(f)=\left(k, v\left(f / h^{k}\right)\right)
$$

for $f \in R_{k} \backslash\{0\}$. Also as before, $\tilde{v}$ has one-dimensional leaves. The following is straightforward.

Lemma 11.2. The valuation $\tilde{v}$ in $(11.2)$ is $\mathbb{H}$-invariant.

Let $S=S(R) \subset \mathbb{N} \times \mathbb{Z}^{n}$ be the semigroup associated to the algebra $R$ and the valuation $\tilde{v}$. As before, we assume that $S(R)$ is finitely generated and that, as a group, $S$ generates all of $\mathbb{Z}^{n+1} \cong \mathbb{Z} \times \mathbb{Z}^{n}$. We record the following Ok96, KaKh-CB.

Lemma 11.3. Let $(k, a) \in S$.

(a) There exists an $\mathbb{H}$-weight vector $f \in R_{k}$ with $\tilde{v}(f)=(k, a)$.

(b) Let $\lambda$ be the $\mathbb{H}$-weight of the vector $f$ found in part (a). The association $(k, a) \mapsto \lambda$ gives a well-defined function which is a semigroup homomorphism $S \rightarrow M$.

Since $S$ generates the group $\mathbb{Z}^{n+1}$, the semigroup homomorphism constructed in Lemma 11.3.(b) extends uniquely to a group homomorphism

$$
\tilde{\lambda}: \mathbb{Z}^{n+1} \rightarrow M .
$$

Let $\lambda=\tilde{\lambda}_{\{0\} \times \mathbb{Z}^{n}}$ denote the restriction of $\tilde{\lambda}$ to $\{0\} \times \mathbb{Z}^{n} \cong \mathbb{Z}^{n}$. As in Section 11.1 we can recover $\tilde{\lambda}$ from its restriction $\lambda$ as follows. Notice that since $\tilde{v}(h)=(1,0)$, we have $\tilde{\lambda}(1,0)=\lambda_{0}$. Then for any $(k, a) \in S$ we have $\tilde{\lambda}(k, a)=\lambda(a)+k \lambda_{0}$. Also as in Section 11.1. we extend $\tilde{\lambda}$ to a linear map $\tilde{\lambda}_{\mathbb{R}}: \mathbb{R}^{n} \rightarrow M_{\mathbb{R}}$. Let $\Lambda_{\mathbb{R}}^{\prime}$ denote the kernel of $\tilde{\lambda}_{\mathbb{R}}$, and let $S^{\prime}$ be the semigroup $S \cap \Lambda_{\mathbb{R}}^{\prime}$. Now consider the subalgebra $R^{\prime}=R^{\mathbb{H}}$, i.e., $R^{\prime}$ is the 0 -graded part of $R$ with respect to the $M$-grading. The following lemma follows readily from Lemmas 11.1 and 11.3 .

Lemma 11.4. The semigroup $S^{\prime}:=S \cap \Lambda_{\mathbb{R}}^{\prime}$ coincides with the semigroup $S\left(R^{\prime}\right)$ associated to the algebra $R^{\prime}$ and the valuation $\tilde{v}$. In particular, $S\left(R^{\prime}\right)$ is finitely generated.

11.3. Integrable systems compatible with GIT and symplectic quotients. We retain the notation of Section 11.2. In particular, $\mathbb{H}$ denotes the torus which acts on $X$ (and hence $R$ ). Since the valuation $\tilde{v}$ on $R$ is $\mathbb{H}$-invariant by Lemma 11.2 the $M$-grading on $R$ is compatible with $\tilde{v}$ in the sense that for $k>0$ and $u \in \mathbb{Z}^{n}$, the spaces $\left(R_{k}\right)_{\geq u}$ and $\left(R_{k}\right)_{>u}$ are $M$-graded. We record the following [An13, Proposition 5.18]. Recall that $\mathcal{R}$ is the family of algebras in Theorem 8.3 which degenerates the coordinate ring $R$ to the coordinate ring of a semigroup algebra $\mathbb{C}[S]$.

Proposition 11.5. The $M$-grading on $R$ can be lifted to an $M$-grading on the family $\mathcal{R}$.

Sketch of proof. We use notation as in the proof of Theorem 8.3 Without loss of generality we may assume that the elements $f_{i j} \in R$ are homogeneous with respect to the $M$-grading. We additionally extend the $M$-grading to $R[t]$ by defining $\operatorname{deg}(t)=0$. Then the $\mathbb{C}[t]-$ algebra $\mathcal{R}$ generated by $t$ and the $\tilde{f}_{i j}=t^{w_{i j}} f_{i j}$ is an $(\mathbb{N} \times M \times \mathbb{N})$-graded subalgebra of $R[t]$. In addition, we can equip $\mathbb{C}\left[x_{i j}, \tau\right]$ with an $M$-grading by defining $\operatorname{deg}(\tau)=0$ and $\operatorname{deg}\left(x_{i j}\right)=\operatorname{deg}\left(f_{i j}\right)=\tilde{\lambda}\left(i, u_{i j}\right) \in M$ where $\tilde{v}\left(f_{i j}\right)=\left(i, u_{i j}\right)$. Then the map $\mathbb{C}\left[x_{i j}, \tau\right] \rightarrow \mathcal{R}$ preserves the $M$-gradings.

Taking Proj we obtain the following. 
Corollary 11.6. (a) The $\mathbb{H}$-action on $X$ lifts to an $\mathbb{H}$-action on the family $\mathfrak{X}$.

(b) The family $\mathfrak{X}$ is an $\mathbb{H}$-invariant subvariety of $W \mathbb{P} \times \mathbb{C}$, where $\mathbb{H}$ acts on $W \mathbb{P}$ via the natural action of $\mathbb{H}$ on $L^{*},\left(L^{2}\right)^{*}, \ldots,\left(L^{r}\right)^{*}$, and $\mathbb{H}$ acts on $\mathbb{C}$ trivially.

As mentioned above, in order to make the constructions of the toric degeneration and the integrable system compatible with the $\mathbb{H}$-action, we must choose the Khovanskii basis $\left\{f_{i j}\right\}$ appropriately. More specifically, we assume the following.

(1) Each $f_{i j}$ is homogeneous with respect to the $M$-grading.

(2) A subset of the collection $\left\{f_{i j}\right\}$ forms a Khovanskii basis for $R^{\prime}$. More precisely, For each $i, 1 \leq i \leq r$, there exists $n_{i}^{\prime} \leq n_{i}$ such that the $\left\{f_{i j}\right\}_{1 \leq j \leq n_{i}^{\prime}}$ are homogeneous of degree 0 with respect to the $M$-grading, and the collection $\left\{\tilde{v}\left(f_{i j}\right)\right\}_{1 \leq i \leq r, 1 \leq j \leq n_{i}^{\prime}}$ generate the semigroup $S\left(R^{\prime}\right)$.

Let $\mathcal{R}$ and $\mathcal{R}^{\prime}$ denote the degenerating families corresponding to $R$ and $R^{\prime}$, respectively, constructed as in Theorem 8.3 using the collections $\left\{f_{i j}\right\}_{1 \leq i \leq r, 1 \leq j \leq n_{i}}$ and $\left\{f_{i j}\right\}_{1 \leq i \leq r, 1 \leq j \leq n_{i}^{\prime}}$ respectively. The following is immediate.

Lemma 11.7. The algebra $\mathcal{R}^{\prime}$ is the degree-0 part of $\mathcal{R}$, i.e. $\mathcal{R}^{\prime}=\mathcal{R}^{\mathbb{H}}$, with respect to the $M$-grading on $\mathcal{R}$ from Proposition 11.5 .

Taking Proj we obtain the following.

Corollary 11.8. There exist flat families $\pi: \mathfrak{X} \rightarrow \mathbb{C}$ and $\pi^{\prime}: \mathfrak{X}^{\prime} \rightarrow \mathbb{C}$ and a morphism $p: \mathfrak{X} \rightarrow \mathfrak{X}^{\prime}$ such that:

(a) The family $\mathfrak{X}$ is an $\mathbb{H}$-variety and the projection $\pi$ is $\mathbb{H}$-invariant.

(b) The family $\mathfrak{X}^{\prime}$ is the GIT quotient $\mathfrak{X} / / \mathbb{H}$ of $\mathfrak{X}$ by $\mathbb{H}$.

(c) Let $\mathfrak{X}^{s s} \subset \mathfrak{X}$ denote the set of semistable points of $\mathfrak{X}$ with respect to the $\mathbb{H}$-action and let $p: \mathfrak{X}^{s s} \rightarrow \mathfrak{X}^{\prime}=\mathfrak{X}^{s s} / \mathbb{H}$ be the quotient map. Then the diagram

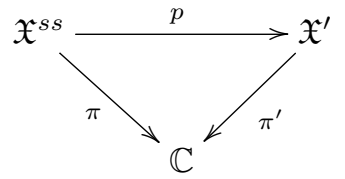

commutes.

(vi) The general fibers of $\mathfrak{X}$ and $\mathfrak{X}^{\prime}$ are $X$ and $X^{\prime}=X / / \mathbb{H}$, respectively, and the special fibers are $X_{0}$ and $X_{0}^{\prime}=X_{0} / / \mathbb{H}$, respectively.

Now let $\mu: X \rightarrow \mathbb{R}^{n}$ and $\mu^{\prime}: X^{\prime} \rightarrow \mathbb{R}^{n-m}$ be the integrable systems constructed in Theorem 5.2 corresponding to the families $\mathfrak{X}$ and $\mathfrak{X}^{\prime}$ respectively. The following is now immediate from Theorem 6.3 and discussion in Section 6 . 
Theorem 11.9. The following diagram is commutative:

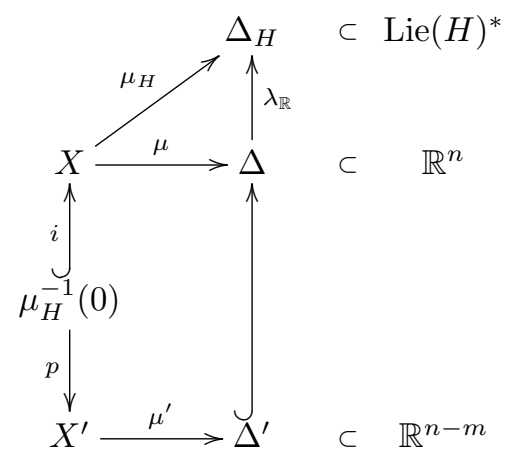

\section{EXAMPLES}

In previous sections we used the notation $\mathbb{H}$ to denote an algebraic torus acting on the variety $X$ where $\operatorname{dim}_{\mathbb{C}} \mathbb{H}=m$ is possibly less than $n=\operatorname{dim}_{\mathbb{C}} X$, and we reserved the notation $\mathbb{T}$ to denote the algebraic torus whose dimension is precisely equal to $\operatorname{dim}_{\mathbb{C}} X$. In the discussion below, we deviate from this notation and use the notation $\mathbb{T}$ (as is standard in the literature) for the torus which acts on $X$, even when $\operatorname{dim}_{\mathbb{C}} \mathbb{T}$ is strictly less than $\operatorname{dim}_{\mathbb{C}} X$.

12.1. Elliptic curves. Let $X$ be an elliptic curve, and $v$ be the valuation on $\mathbb{C}(X)$ associated to a point $p \in X$. Consider the line bundle $\mathcal{L}=\mathcal{O}_{X}(3 p)$ and $L=H^{0}\left(X, \mathcal{O}_{X}(3 p)\right)$ giving the cubic embedding of $X$ in $\mathbb{C P}^{2}$. The semigroup $S(R(L)) \subset \mathbb{N} \times \mathbb{Z}$ is generated by $(1,0),(1,1),(1,3)$ and hence finitely generated An13, Example 5]. The curve $X$ degenerates to a cuspidal cubic curve. The Newton-Okounkov body $\Delta(R(L))$ is the line segment $[0,3]$. Theorem 10.1 gives a function $F: X \rightarrow \mathbb{R}$ which is continuous on all of $X$, differentiable on a dense open subset $U$, with image precisely $[0,3]$, and whose Hamiltonian vector field generates a circle action on $U$. Here $U$ is the complement of the figure 8 in $X$. See Figure 1 . Moreover, the inverse image of the open interval $(0,3)$ is contained in $U$.

12.2. Flag varieties. Let $G$ be a connected complex reductive algebraic group, $B$ a Borel subgroup and $\mathbb{T}$ its maximal torus. We denote the weight lattice, i.e. the character lattice of $\mathbb{T}$, by $\Lambda$. Then $\Lambda^{+}$(respectively $\Lambda_{\mathbb{R}}^{+}$) are the semigroup of dominant weights (respectively positive Weyl chamber) corresponding to the choice of $B$. We also fix a maximal compact subgroup $K$ compatible with the choice of $B$ and $\mathbb{T}$ so that $T=K \cap \mathbb{T}$ is a maximal torus of $K$.

Let $G / B$ denote the complete flag variety of $G$. Given a regular dominant weight $\lambda$, i.e. a weight in the interior of the positive Weyl chamber, the variety $G / B$ embeds in the projective space $\mathbb{P}\left(V_{\lambda}\right)$ as the $G$-orbit of a highest weight vector. Here $V_{\lambda}$ is the irreducible $G$-module with highest weight $\lambda$. More generally, let $\lambda$ be a dominant weight (possibly on the boundary of the positive Weyl chamber). Then the $G$-orbit of a highest weight vector $v_{\lambda}$ in the projective space $\mathbb{P}\left(V_{\lambda}\right)$ is a partial flag variety $X_{\lambda}=G / P_{\lambda}$ where $P_{\lambda}$ is the $G$ stabilizer of $v_{\lambda}$ in $\mathbb{P}\left(V_{\lambda}\right)$. Let $\mathcal{L}_{\lambda}$ be the restriction of the line bundle $\mathcal{O}(1)$ on the projective space $\mathbb{P}\left(V_{\lambda}\right)$ to $X_{\lambda}$. By the Borel-Weil-Bott theorem the space of sections $H^{0}\left(X_{\lambda}, \mathcal{L}_{\lambda}\right)$ is isomorphic to $V_{\lambda}^{*}$ as a $G$-module.

Let $N=\operatorname{dim}_{\mathbb{C}}(G / B)$. The so-called string polytope is a rational polytope in $\mathbb{R}^{N}$ such that the number of integral points in the polytope is equal to $\operatorname{dim}_{\mathbb{C}}\left(V_{\lambda}\right)$. In fact, more is true: the integral points in a string polytope parameterize the so-called crystal basis for the 

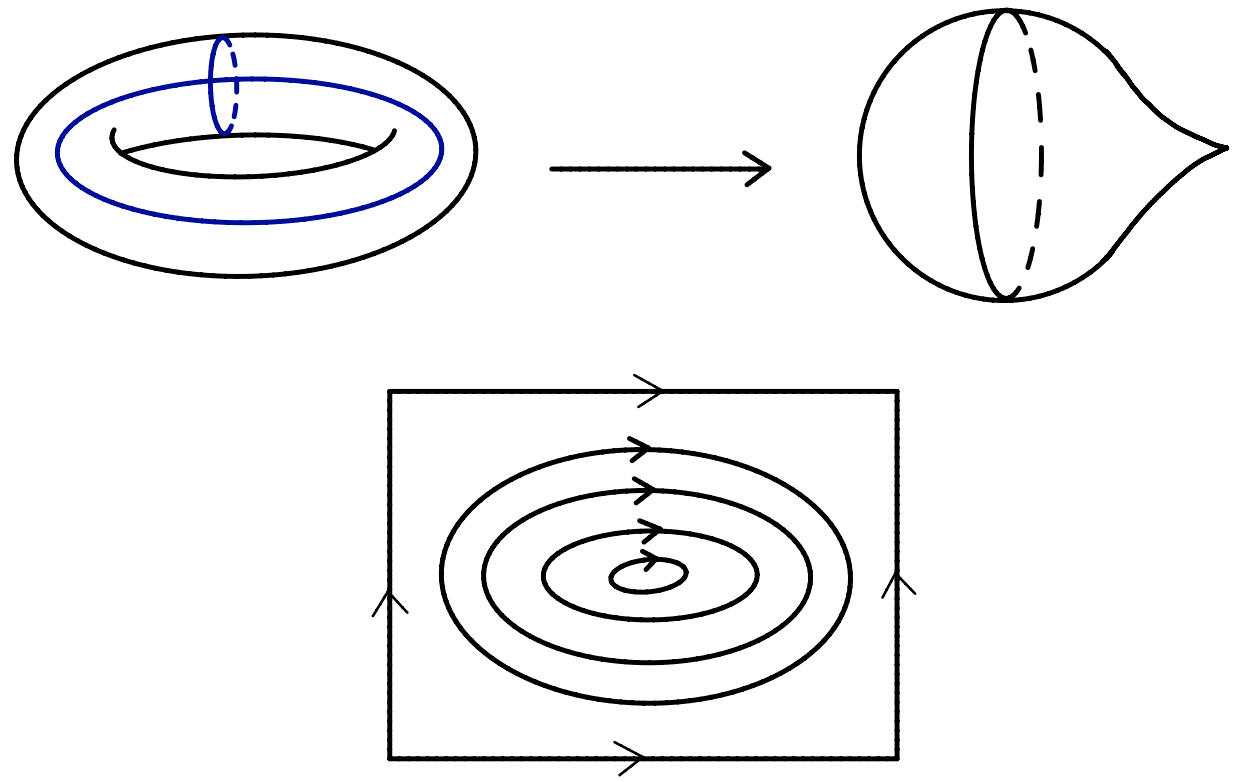

Figure 1. The elliptic curve degenerates to a cuspidal cubic curve (which is singular). The Hamiltonian $S^{1}$-action on the smooth points of the cuspidal cubic is the usual rotation; this pulls back to an $S^{1}$-action on the complement of the figure 8 in the elliptic curve.

$G$-module $V_{\lambda}$ (see [Li98). The construction of the string polytope depends on the choice of a reduced word decomposition $\underline{w}_{0}=\left(s_{i_{1}}, s_{i_{2}}, \ldots, s_{i_{N}}\right)$ for the longest element $w_{0}$ in the Weyl group $W$ of $(G, T)$, where here the $s_{i}$ denote the simple reflections corresponding to the simple roots $\alpha_{i}$. Thus, the string polytope associated to a dominant weight $\lambda$ and a reduced decomposition $\underline{w}_{0}$ is often denoted as $\Delta_{\underline{w}_{0}}(\lambda)$ [BeZe01, Li98].

Remark 12.1. The well-known Gel'fand-Cetlin polytopes GeCe50 corresponding to irreducible representations of $\mathrm{GL}(n, \mathbb{C})$ are special cases of the string polytopes. More precisely, let $G=\mathrm{GL}(n, \mathbb{C})$. Here the Weyl group is $W=S_{n}$. Choose the reduced word decomposition

$$
w_{0}=\left(s_{1}\right)\left(s_{2} s_{1}\right)\left(s_{3} s_{2} s_{1}\right) \cdots\left(s_{n-1} \cdots s_{1}\right)
$$

for the longest element $w_{0} \in S_{n}$, where $s_{i}$ denotes the simple transposition exchanging $i$ and $i+1$. Then $\Delta_{\underline{w}_{0}}(\lambda)$ can be identified (after a linear change of coordinates) with the Gel'fand-Cetlin polytope corresponding to $\lambda$. Similarly, for $G=\operatorname{Sp}(2 n, \mathbb{C})$ or $\operatorname{SO}(n, \mathbb{C})$, for analogous well-chosen reduced decompositions of the corresponding longest elements of the Weyl group, we can recover the corresponding Gel'fand-Cetlin polytopes as string polytopes Li98.

In fact, given a reduced decomposition $\underline{w}_{0}$, there is a rational polyhedral cone $\mathcal{C}_{\underline{w}_{0}}$ in $\Lambda_{\mathbb{R}}^{+} \times \mathbb{R}^{N}$ such that each string polytope $\Delta_{\underline{w}_{0}}(\lambda)$ is the slice of the cone $\mathcal{C}_{\underline{w}_{0}}$ at $\lambda$, i.e., $\Delta_{\underline{w}_{0}}(\lambda)=\mathcal{C}_{\underline{w}_{0}} \cap \pi^{-1}(\lambda)$ where $\pi: \Lambda_{\mathbb{R}}^{+} \times \mathbb{R}^{N} \rightarrow \Lambda_{\mathbb{R}}^{+}$is the projection on the first factor [Li98].

We note the following. 
(1) We can define the polytope $\Delta_{\underline{w}_{0}}(\lambda)=\mathcal{C}_{\underline{w}_{0}} \cap \pi^{-1}(\lambda)$ for any $\lambda \in \Lambda_{\mathbb{R}}^{+}$.

(2) The fact that $\mathcal{C}_{\underline{w}_{0}}$ is a convex cone implies that $\Delta_{\underline{w}_{0}}(k \lambda)=k \Delta_{\underline{w}_{0}}(\lambda)$ for any $k>0$. Moreover, for $\lambda_{1}, \lambda_{2} \in \Lambda_{\mathbb{R}}^{+}$we have $\Delta_{\underline{w}_{0}}\left(\lambda_{1}\right)+\Delta_{\underline{w}_{0}}\left(\lambda_{2}\right) \subset \Delta_{\underline{w}_{0}}\left(\lambda_{1}+\lambda_{2}\right)$.

(3) One also proves that the map:

$$
\pi_{\lambda}:\left(t_{1}, \ldots, t_{N}\right) \mapsto-\lambda+t_{1} \alpha_{i_{1}}+\cdots+t_{N} \alpha_{i_{N}},
$$

projects the string polytope $\Delta_{\underline{w}_{0}}(\lambda)$ onto the polytope $P(\lambda)$ which is the convex hull of the Weyl group orbit of $\bar{\lambda}$.

In Ca02 Caldero constructs a flat deformation of the flag variety $X_{\lambda}$ to the toric variety $X_{\lambda, w_{0}}$ corresponding to the string polytope $\Delta_{\underline{w}_{0}}(\lambda)$. The key ingredient in his construction is a multiplicativity property of the (dual) canonical basis with respect to the string parametrization. In Ka11 it is shown that Anderson's toric degeneration recounted in Section 8 is a generalization of Caldero's construction.

Fix a $K$-invariant Hermitian metric on $\mathbb{P}\left(V_{\lambda}\right)$. Let $\mu_{T}$ denote the moment map for the Hamiltonian action of the compact torus $T \subset K$ on $X_{\lambda} \cong G / P_{\lambda}$. Theorem 10.1 applied to $\left(X_{\lambda}, \mathcal{L}_{\lambda}\right)$ imply the following:

Corollary 12.2. Let $n=\operatorname{dim}_{\mathbb{C}}\left(X_{\lambda}\right)$. There exists an integrable system $\mu_{\lambda}=\left(F_{1}, \ldots, F_{n}\right)$ : $X_{\lambda} \rightarrow \mathbb{R}^{n}$ (in the sense of Definition 1.1) such that

(a) the image of $\mu_{\lambda}$ is the string polytope $\Delta_{\underline{w}_{0}}(\lambda)$, and

(b) the diagram

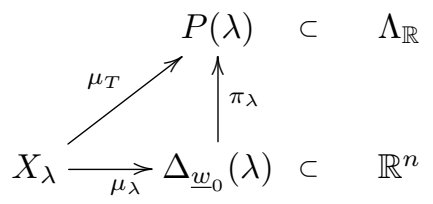

commutes, where the vertical arrow $\pi_{\lambda}$ is the linear projection given in (12.1).

(c) Moreover, the functions $F_{1}, \ldots, F_{n}$ generate a torus action on the open dense subset $U$, on which the $F_{i}$ are differentiable and Poisson commute, and the inverse image of the interior of $\Delta$ is contained in $U$.

12.3. Spherical varieties. In fact, the constructions and results in Section 12.2 can be extended to the larger class of spherical varieties. Spherical varieties are the algebraic analogues of multiplicity-free Hamiltonian spaces. More precisely, let $G$ be a connected reductive algebraic group. Let $X$ be a normal algebraic variety equipped with an algebraic $G$-action. Then $X$ is called spherical if a (and hence any) Borel subgroup of $G$ has a dense open orbit. Flag varieties $G / P_{\lambda}$ and $\mathbb{T}$-toric varieties are examples of spherical varieties, with respect to the actions of $G$ and $\mathbb{T}$, respectively.

Let $X$ be a normal projective spherical $G$-variety of dimension $n$ and $\mathcal{L}$ a $G$-linearized very ample line bundle. Then $X$ embeds $G$-equivariantly in the projective space $\mathbb{P}(V)$ where $V=H^{0}(X, \mathcal{L})^{*}$. Let $K$ denote a maximal compact subgroup of $G$ as in the previous section, and fix a $K$-invariant Hermitian product on $V$. This induces a Kähler metric on $\mathbb{P}(V)$ and hence on the smooth locus of $X$. With respect to the corresponding symplectic structure, (the smooth locus of) $X$ is a Hamiltonian $K$-space; let $\mu_{K}$ denote the moment map. The Kirwan polytope, denoted $Q(X, \mathcal{L})$, is defined to be the intersection of the moment map image of $\mu_{K}$ with the positive Weyl chamber of $\operatorname{Lie}(K)^{*}$. 
For this discussion we fix a reduced decomposition $\underline{w}_{0}$ for the longest element $w_{0}$. In Ok97] and [AlBr04 the authors introduce a polytope $\Delta_{\underline{w}_{0}}(X, \mathcal{L}) \subset \Lambda_{\mathbb{R}}^{+} \times \mathbb{R}^{N}$ defined as

$$
\Delta_{\underline{w}_{0}}(X, \mathcal{L}):=\left\{(\lambda, x) \mid \lambda \in Q(X, \mathcal{L}), x \in \Delta_{\underline{w}_{0}}(\lambda)\right\} .
$$

That is, $\Delta_{\underline{w}_{0}}(X, \mathcal{L})$ is the polytope fibered over the moment polytope $Q(X, \mathcal{L})$ with the string polytopes as fibers. We call the polytope $\Delta_{\underline{w}_{0}}(X, \mathcal{L})$ the string polytope of the spherical variety $X$. In $\mathrm{AlBr} 04$ and $\mathrm{Ka05}$ it is shown that there is a flat degeneration of $X$ to the toric variety associated to the rational polytope $\Delta_{\underline{w}_{0}}(X, \mathcal{L})$. In fact, in Ka11 it is shown that, with respect to certain choices of valuations, the string polytopes $\Delta_{\underline{w}_{0}}(\lambda)$ and $\Delta_{\underline{w}_{0}}(X, \mathcal{L})$ can be realized as Newton-Okounkov bodies, and the degenertions in Ca02, AlBr04] and Ka05] all fit into the general toric degeneration framework discussed in Section 8 .

Generalizing the case of the flag varieties discussed in Section 12.2 we have the following. Let $P(X, \mathcal{L})$ denote the polytope which is the convex hull of the $W$-orbit of the Kirwan polytope $Q(X, \mathcal{L})$. It can be shown that $P(X, \mathcal{L})$ is precisely the moment polytope for the Hamiltonian $T$-action on $X$. Let $\mu_{T}$ denote the moment map for this action. Applying Theorem 10.1 to $(X, \mathcal{L})$, we obtain the following.

Theorem 12.3. Let $X$ and $\mathcal{L}$ be as above. Let $\operatorname{dim}_{\mathbb{C}} X=n$. Then there exists an integrable system $\mu=\left(F_{1}, \ldots, F_{n}\right): X \rightarrow \mathbb{R}^{n}$ (in the sense of Definition 1.1) such that

(a) the image of $\mu$ can be identified with the string polytope $\Delta_{\underline{w}_{0}}(X, \mathcal{L})$, and

(b) the diagram

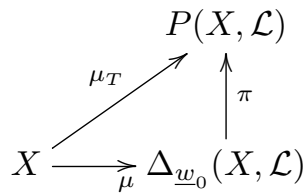

commutes, where the vertical arrow $\pi$ is the linear projection given in (12.1).

(c) Moreover, the functions $F_{1}, \ldots, F_{n}$ generate a torus action on the open dense subset $U$, on which the $F_{i}$ are differentiable and Poisson commute, and the inverse image of the interior of $\Delta$ is contained in $U$.

Remark 12.4. Let $X$ be a smooth spherical $G$-variety of dimension $n$ equipped with a $G$ linearized very ample line bundle $\mathcal{L}$. It can be shown that the space of smooth $K$-invariant functions on $X$ is commutative, in the sense that any two smooth $K$-invariant functions Poisson-commute. We believe that the integrable system $\mu=\left(F_{1}, \ldots, F_{n}\right)$ in Theorem 12.3 can be constructed so that $\left(F_{1}, \ldots, F_{r}\right)$ are smooth $K$-invariant functions on all of $X$, where $r \leq n$ is the so-called rank of the spherical variety (i.e., the minimal codimension of a $K$-orbit).

12.4. Weight varieties. We maintain the notation of Section 12.2 Let $P(\lambda)$ denote the convex hull of the $W$-orbit of $\lambda$. Recall that a weight variety $X_{\lambda, \gamma}$ is the GIT quotient of $X_{\lambda}$ by the action of $\mathbb{T}$ twisted by an integral weight $\gamma \in P(\lambda) \cap \Lambda^{+}$. More precisely, let $\gamma \in P(\lambda) \cap \Lambda^{+}$be a character of $\mathbb{T}$ and $\mathcal{L}_{\lambda}(-\gamma)$ be the $\mathbb{T}$-line bundle $\mathcal{L}_{\lambda}$ where the action of $\mathbb{T}$ is twisted by $-\gamma$. Then one defines

$$
X_{\lambda, \gamma}:=X_{\lambda}^{s s}\left(\mathcal{L}_{\lambda}(-\gamma)\right) / \mathbb{T} \text {. }
$$

Important examples of weight varieties are polygon spaces [HaKn97]. 
An alternative description is as follows. Let $V_{\lambda}^{(\gamma)}$ denote the $\gamma$-weight space in the $G$ module $V_{\lambda}$. The weight variety $X_{\lambda, \gamma}$ is $\operatorname{Proj}\left(R_{\lambda}^{(\gamma)}\right)$ where

$$
R_{\lambda}^{(\gamma)}:=\bigoplus_{k}\left(V_{k \lambda}^{*}\right)^{(k \gamma)}
$$

In other words, $R_{\lambda}^{(\gamma)}$ is the $\mathbb{T}$-invariant subalgebra of $R\left(L_{\lambda}\right)$ for the $(-\gamma)$-twisted action of $\mathbb{T}$ on $R\left(L_{\lambda}\right)$, defined by

$$
t *_{\gamma} f:=\gamma(t)^{-k}(t \cdot f) \quad \forall t \in \mathbb{T}, \forall f \in L^{k} .
$$

When $\gamma$ is a regular value for the moment map $\mu_{T}$, and $T$ acts freely on $\mu_{T}^{-1}(0)$, the variety $X_{\lambda, \gamma}$ can also be identified with the symplectic quotient of $X_{\lambda}$ at the value $\gamma$.

Using Theorem 11.8 we can now recover the following theorem of Foth and $\mathrm{Hu}$ [FoHu05].

Theorem 12.5. There exists a flat degeneration of the weight variety $X_{\lambda, \gamma}$ to a projective toric variety $X_{\lambda, \gamma, 0}$ corresponding to the polytope

$$
\Delta_{\underline{w}_{0}}(\lambda, \gamma)=\Delta_{\underline{w}_{0}}(\lambda) \cap \pi_{\lambda}^{-1}(\gamma),
$$

obtained by slicing the string polytope $\Delta_{\underline{w}_{0}}(\lambda)$ at $\gamma$. Here the projection $\pi_{\lambda}$ is that given in (12.1).

As in Section 6, the GIT quotient $X_{\lambda, \gamma}$ inherits a Kähler structure from $X_{\lambda}$. Let $n^{\prime}=$ $\operatorname{dim}_{\mathbb{C}} X_{\lambda, \gamma}$. From Corollary 12.2 and Theorem 11.9 we obtain the following.

Corollary 12.6. Under the assumptions and notation as above, there exists an integrable system $\mu_{\lambda, \gamma}=\left(F_{1}, \ldots, F_{n^{\prime}}\right)$ on $X_{\lambda, \gamma}$ (in the sense of Definition 1.1) such that

(a) the image of $\mu_{\lambda, \gamma}$ is the string polytope $\Delta_{\underline{w}_{0}}(\lambda, \gamma)$, and

(b) the diagram

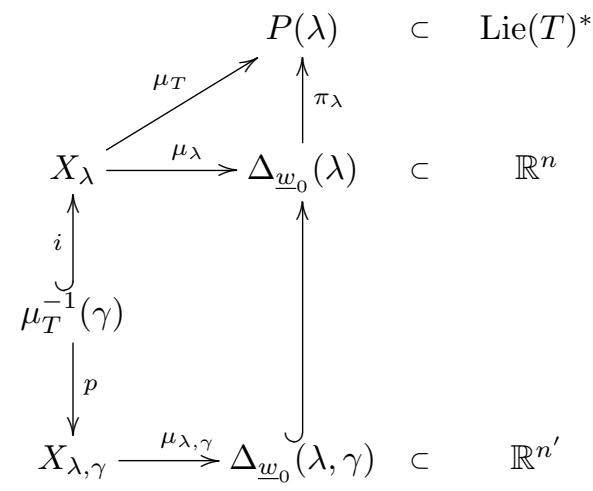

commutes, where the map $\mathbb{R}^{n^{\prime}} \hookrightarrow \mathbb{R}^{n}$ is the inclusion of the first $n^{\prime}$ coordinates.

\section{REFERENCES}

[AlBr04] V. Alexeev and M. Brion, M. Toric degeneration of spherical varities. Selecta Math. (N.S.) 10 (2004), no. 4, 453-478.

[An13] D. Anderson. Okounkov bodies and toric degenerations. Math. Ann. 356 (2013), no. 3, 1183-1202.

[AKL12] D. Anderson, A. Kuronya and V. Lozovanu. Okounkov bodies of finitely generated divisors. Int. Math. Res. Not. IMRN (2014), no. 9, 2343-2355.

[Au96] M. Audin. Spinning Tops, A course on integrable systems. Cambridge Studies in Advanced Mathematics, 51. Cambridge University Press, Cambridge, 1996. 
[BeZe01] A. Berenstein and A. Zelevinsky. Tensor product multiplicities, canonical bases and totally positive varieties. Invent. Math. 143 (2001), no. 1, 77-128.

[Br86] M. Brion. Sur l'image de l'application moment. Séminaire d'algèbre Paul Dubreil et Marie-Paule Malliavin (Paris, 1986), Springer Lecture Notes in Math., 1296 (1987), 177-192.

[BG09] W. Bruns and J. Gubeladze. Polytopes, rings, and K-theory Springer, Dordrecht, 2009.

[Bu10] D. Burns. Toric Degenerations and the Exact Bohr-Sommerfeld Correspondence Preprint (2010).

[Ca02] P. Caldero. Toric degenerations of Schubert varieties. Transform. Groups 7 (2002), no. 1, 51-60.

[CoLiSc11] D. Cox and J. Little and H. Schenck. Toric varieties. Graduate Studies in Mathematics, 124. American Mathematical Society, Providence, RI, 2011.

[Do82] I. Dolgachev. Weighted projective varieties. Group actions and vector fields (Vancouver, B.C., 1981), 3471, Lecture Notes in Math., 956, Springer, Berlin, 1982.

[Ei95] D. Eisenbud. Commutative algebra. with a view toward algebraic geometry. Graduate Texts in Mathematics, 150. Springer-Verlag, New York, 1995.

[FoHu05] P. Foth and Y. Hu. Toric degeneration of weight varieties and applications. Travaux Mathématiques, Volume 16 (2005), 87 - 105.

[GeCe50] I.M. Gel'fand and M.L. Cetlin. Finite dimensional representations of the group of unimodular matrices. Doklady Akad. Nauk USSR (N.S.), 71 (1950), 825-828.

[GoLa96] N. Gonciulea and V. Lakshmibai. Degenerations of flag and Schubert varieties to toric varieties. Transformation Groups, Volume 1 (1996), no. 3, 215-248.

[GrHa78] P. Griffiths and J. Harris. Principles of algebraic geometry. Pure and Applied Mathematics. WileyInterscience [John Wiley \& Sons], New York, 1978.

[GuSt83] V. Guillemin and S. Sternberg. The Gel'fand-Cetlin system and quantization of the complex flag manifolds. Journal of Functional Analysis, 52 (1983), 106-128.

[HaKo11] M. Hamilton and H. Konno. Convergence of Kähler to real polarizations on flag manifolds via toric degenerations. Journal of Symplectic Geometry, 12 (2014) no. 3, 473-509.

[Ha77] R. Hartshorne. Algebraic geometry. Graduate Texts in Mathematics, 52. Springer-Verlag, New York, 1977.

[HaKn97] J-C. Hausmann and A. Knutson. Polygon spaces and Grassmannians. L'enseignement Mathématique, 43 (1997), 173-198.

[HuLi05] J. Hu and W.-P. Li. Theory of ordinary differential equations: existence, uniqueness and stability. Online notes: http://www.math.ust.hk/ majhu/Math4051/Notes.pdf

[JeWe] L. Jeffrey and J. Weitsman. Bohr-Sommerfeld orbits in the moduli space of flat connections and the Verlinde dimension formula. Comm. Math. Phys. 150 (1992), no. 3, 593-630.

[KaTo05] Y. Karshon and S. Tolman. The Gromov width of complex Grassmannians. Algebr. Geom. Topol. 5 (2005), 911-922.

[Ka05] K. Kaveh. SAGBI bases and degeneration of spherical varieties to toric varieties. Michigan Math. J. 53 (2005), no. 1, 109-121.

[Ka11] K. Kaveh. Crystal bases and Newton-Okounkov bodies, arXiv:1101.1687. To appear in Duke Mathematical Journal.

[KaKh08] K. Kaveh and A. Khovanskii. Convex bodies and algebraic equations on affine varieties, arXiv:0804.4095v1. A short version with title Algebraic equations and convex bodies appeared in Perspectives in Analysis, Topology and Geometry, Birkhäser series Progress in Mathematics.

[KaKh10] K. Kaveh and A. Khovanskii. Mixed volume and an extension of intersection theory of divisors. Moscow Math. J. 10 (2010), no. 2, 343-375

[KaKh-NO] K. Kaveh and A. Khovanskii. Newton-Okounkov bodies, semigroups of integral points, graded algebras and intersection theory. Ann. of Math. 176 (2012), no. 2, 925-978.

[KaKh-CB] K. Kaveh and A. Khovanskii. Convex bodies associated to actions of reductive groups. Moscow Math. J. 12 (2012), no. 2, 369-396, 461 (volume in honor of V. I. Arnold).

[KoMi05] M. Kogan and E. Miller. Toric degeneration of Schubert varieties and Gelfand-Tsetlin polytopes. Adv. Math. 193 (2005), no. 1, 1-17.

[KnTr-MO] Math Overflow posts by David Treumann and Allen Knutson, 2009 and 2010. http:// mathoverflow.net/questions/2203/

[KuMoPa00] K. Kurdyka and T. Mostowski and A. Parusiński. Proof of the gradient conjecture of R. Thom. Ann. of Math. (2), 152 (2000), no. 3, 763-792.

[Ku94] K. Kurdyka and A. Parusinski. $w_{f}$-stratification of subanalytic functions and the Łojasiewicz inequality. C. R. Acad. Sci. Paris Ser. I Math. 318 (1994), no. 2, 129133. 
[LaMu09] R. Lazarsfeld and M. Mustata. Convex bodies associated to linear series. Ann. Sci. Ec. Norm. Super. (4) 42 (2009), no. 5, 783-835.

[Lerman] E. Lerman. Gradient flow of the norm-square of the moment map. L'Enseign. Math. (2) 51 (2005), no. $1-2,117-127$.

[Li98] P. Littelmann. Cones, crystals, and patterns. Transform. Groups 3 (1998), no. 2, 145-179.

[Lo84] S. Łojasiewicz. Sur les trajectoires du gradient d'une fonction analytique. Geometry seminars, 19821983 (Bologna, 1982/1983), 115-117. Univ. Stud. Bologna, Bologna, 1984.

[Ma11] C. Manon. Toric degenerations and tropical geometry of branching algebras, arxiv: 1103.2484.

[Mu76] D. Mumford. Algebraic geometry. I. Complex projective varieties. Grundlehren der Mathematischen Wissenschaften, No. 221. Springer-Verlag, Berlin-New York, 1976.

[MuFoKi94] D. Mumford and J. Fogarty and F. Kirwan. Geometric invariant theory. Third edition. Ergebnisse der Mathematik und ihrer Grenzgebiete (2), 34. Springer-Verlag, Berlin, 1994.

[NiNoUe10] T. Nishinou, Y. Nohara and K. Ueda. Toric degenerations of Gelfand-Cetlin systems and potential functions. Adv. Math. 224 (2010), no. 2, 648-706.

[Ok96] A. Okounkov. Brunn-Minkowski inequality for multiplicities. Invent. Math. 125 (1996), no. 3, 405411.

[Ok97] A. Okounkov. A remark on the Hilbert polynomial of a spherical variety. Func. Anal. and Appl., 31 (1997), 82-85.

[Ok00] A. Okounkov. Why would multiplicities be log-concave? The orbit method in geometry and physics (Marseille, 2000), 329-347, Progr. Math., 213, Birkha"user Boston, Boston, MA, 2003.

[PeVu11] A. Pelayo and S. Vu Ngoc. Symplectic theory of completely integrable Hamiltonian systems. Bull. Amer. Math. Soc. (N.S.), Vol. 48 (2011), 409-455.

[Ru99] W.-D. Ruan. Lagrangian torus fibration of quintic hypersurfaces. I. Fermat quintic case. In Winter School on Mirror Symmetry, Vector Bundles and Lagrangian Submanifolds (Cambridge, MA, 1999), volume 23 of AMS/IP Studies in Advanced Mathematics, pp. 297-332. Amer. Math. Soc., Providence, RI, 2001.

[Te99] B. Teissier. Valuations, deformations, and toric geometry. In Valuation theory and its applications, Vol. II (Saskatoon, SK, 1999), 361-459, Fields Inst. Commun., 33, Amer. Math. Soc., Providence, RI, 2003.

Department of Mathematics and Statistics, McMaster University, 1280 Main Street West, Hamilton, Ontario L8S4K1, Canada

E-mail address: Megumi.Harada@math.mcmaster.ca

$U R L:$ http://www.math.mcmaster.ca/Megumi.Harada

Department of Mathematics, School of Arts and Sciences, University of Pittsburgh, 301

Thackeray Hall, Pittsburgh, PA 15260, U.S.A.

E-mail address: kaveh@pitt.edu

$U R L:$ http://www.pitt.edu/ kaveh 